Homology, Homotopy and Applications, vol.9(2), 2007, pp.19-94

\title{
BRAID COBORDISMS, TRIANGULATED CATEGORIES, AND FLAG VARIETIES
}

\author{
MIKHAIL KHOVANOV AND RICHARD THOMAS \\ (communicated by Ralph Cohen)
}

\begin{abstract}
We argue that various braid group actions on triangulated categories should be extended to projective actions of the category of braid cobordisms and illustrate how this works in examples. We construct actions of both the affine braid group and the braid cobordism category on the derived category of coherent sheaves on the cotangent bundle to the full flag variety.
\end{abstract}

\section{Introduction}

2 Examples of actions of braid groups and braid cobordisms on triangulated categories

3 Invariants of braid cobordisms $\quad 31$

3.1 Braid cobordisms and braid group categorifications . . . . . . . . . 31

3.2 Categorification from the rings $A_{n} \ldots \ldots \ldots \ldots$

3.3 Categorification via Rouquier complexes . . . . . . . . . . . . 45

3.4 Categorification in Fukaya-Floer categories . . . . . . . . . . . . . . 49

3.5 More on polarization . . . . . . . . . . . . . . . . . . 53

3.6 When Definition 3.3 fails . . . . . . . . . . . . . . . . 54

$4 \quad$ An affine braid group action on $D^{b}\left(T^{*} F l\right) \quad \mathbf{5 5}$

4.1 Notation and statement of results . . . . . . . . . . . . . . 55

4.2 Fourier-Mukai transforms . . . . . . . . . . . . . . . . . 56

4.3 First results . . . . . . . . . . . . . . . . . . . . 62

4.4 The Yang-Baxter equation . . . . . . . . . . . . . . . . . 66

4.5 Extension to the affine braid group . . . . . . . . . . . . 75

5 Actions of the braid cobordism category on derived categories of $\begin{array}{ll}\text { coherent sheaves } & \mathbf{7 7}\end{array}$

5.1 Surfaces with chains of rational curves . . . . . . . . . . . . 77

5.2 The cotangent bundle of the flag variety . . . . . . . . . . . 88

Received October 14, 2006, revised May 23, 2007; published on July 24, 2007.

2000 Mathematics Subject Classification: 18E30.

Key words and phrases: Derived categories, triangulated categories, braid group actions, braid cobordisms, categorification, flag varieties, Fukaya categories, mirror symmetry.

Copyright (c) 2007, International Press. Permission to copy for private use granted. 
Homology, Homotopy and Applications, vol. 9(2), 2007

\section{Introduction}

There are many known and conjectural braid group actions on triangulated categories, including

- Actions generated by a chain of spherical objects [KS, ST, RZ, HK].

- Action on the derived categories of (constructible) sheaves of vector spaces on flag varieties $\left[\mathbf{R o 1}\right.$, Ro3], and related actions on $D^{b}\left(\mathcal{O}_{0}\right)$, where $\mathcal{O}_{0}$ is a regular block of the highest weight category for $\mathfrak{s l}_{n}$, and on its subcategories [St2].

- Actions on categories of complexes of matrix factorizations $[\mathbf{K R}]$.

- Actions on Fukaya-Floer categories of various symplectic manifolds, such as quiver varieties $[\mathbf{S S}]$.

- Actions on derived categories of coherent sheaves on quiver varieties and other Calabi-Yau manifolds.

We begin by giving a brief survey of these and related actions in Sections 2 and 3 . This is meant to convey the diverse areas in which braid group actions arise, but it is not necessary to understand or even read these sections to follow the rest of the paper. By a weak action of a group $G$ on a category $\mathcal{C}$ we mean an assignment of an invertible functor $F_{g}: \mathcal{C} \longrightarrow \mathcal{C}$ to each $g \in G$ such that $F_{g} \circ F_{h} \cong F_{g h}$ for all $g, h \in G$. It follows that $F_{1} \cong \mathrm{Id}$, where $1 \in G$ is the unit element. A weak action is upgraded to a (genuine) action if the functor isomorphisms $F_{g} \circ F_{h} \cong F_{g h}$ are selected to satisfy the associativity constraint, which says that all diagrams below are commutative

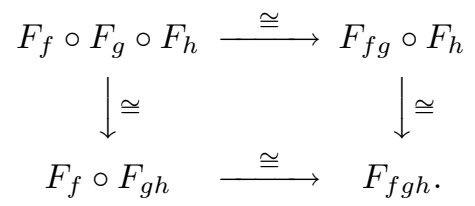

Deligne [De] was the first to discuss braid group action on categories, implicitly emphasizing the difference between weak and genuine actions, and gave a neat criterion for when a weak action of a braid group lifts to a genuine action. Some of the braid group actions reviewed below are genuine and the others are believed to be genuine.

An action of a group $G$ by exact functors on a triangulated category $\mathcal{C}$ induces an action of $G$ on the Grothendieck group $K(\mathcal{C})$. The exact functor $F_{g}$ descends to an endomorphism, denoted $\left[F_{g}\right]$, of the abelian group $K(\mathcal{C})$. Isomorphisms $F_{g} \circ F_{h}$ $\cong F_{g h}$ become equalities between maps $\left[F_{g}\right]\left[F_{h}\right]=\left[F_{g h}\right]$ and we obtain an action of $G$ on $K(\mathcal{C})$. Often $K(\mathcal{C})$ is a free abelian group and, after tensoring it and all maps $\left[F_{g}\right]$ with $\mathbb{C}$, we get a complex representation of $G$, an action of $G$ on the complex vector space $K(\mathcal{C}) \otimes \mathbb{C}$. The operator in $K(\mathcal{C}) \otimes \mathbb{C}$ associated with $g$ is still denoted $\left[F_{g}\right]$. In some cases (for instance, when $\mathcal{C}$ is a triangulated category of complexes of graded modules over a graded ring), $K(\mathcal{C})$ is naturally a $\mathbb{Z}\left[q, q^{-1}\right]$-module, and $\left[F_{g}\right]$ are module maps. Then, to get a complex representation, we tensor $K(\mathcal{C})$ with $\mathbb{C}$ over $\mathbb{Z}\left[q, q^{-1}\right]$, selecting $q \in \mathbb{C}$ to be a generic complex number. Alternatively, one can work with the $\mathbb{Z}\left[q, q^{-1}\right]$-module $K(\mathcal{C})$ itself. 
In this paper we specialize to the case when $G$ is the $n$-stranded braid group $\mathrm{Br}_{n}$. Quite a few well-known complex representations of the braid group can be lifted to actions of $\mathrm{Br}_{n}$ on triangulated categories, including the Burau representation $[\mathbf{K S}, \mathbf{S T}]$, representations that factor through various representations of the Hecke algebra of the symmetric group (by restricting the braid group action on the homotopy category of a regular block of $\mathcal{O}$ for $\mathfrak{s l}_{n}$ to the subcategories considered in $[\mathbf{K M S}]$ ), their $q=1$ specializations, etc.

The existence of such actions of $\mathrm{Br}_{n}$ on triangulated categories is nontrivial, and their potential remains underexplored. The synthesis of the braid group, a manifestly topological object, and triangulated categories, which are strongly rooted in algebra, looks unusual and perplexing. What can one do with this symbiosis?

Suppose we are given a braid group action on $\mathcal{C}$. To each braid $g$ there is associated a functor $F_{g}$. Given two elements $g, h$ of the braid group, we can form the set of natural transformations $\operatorname{Hom}\left(F_{g}, F_{h}\right)$ from $F_{g}$ to $F_{h}$ in $\mathcal{C}$. Together, they form a monoidal category with elements of $\mathrm{Br}_{n}$ as objects and $\operatorname{Hom}\left(F_{g}, F_{h}\right)$ as morphisms from $g$ to $h$. This information about the braid group action on $\mathcal{C}$ is lost upon passing to the Grothendieck group.

At the same time, there exists a monoidal category of purely topological origin with elements of the braid group $\mathrm{Br}_{n}$ as objects. We call it the category of braid cobordisms and denote it by $\mathcal{B C}_{n}$.

We can think of an $n$-stranded braid as a cobordism from $n$ fixed points on a plane to itself, such that the projection onto the vertical direction has no critical points; see below. Isotopy classes of $n$-stranded braids constitute the braid group $\mathrm{Br}_{n}$. Sometimes, isotopy classes are themselves called braids.

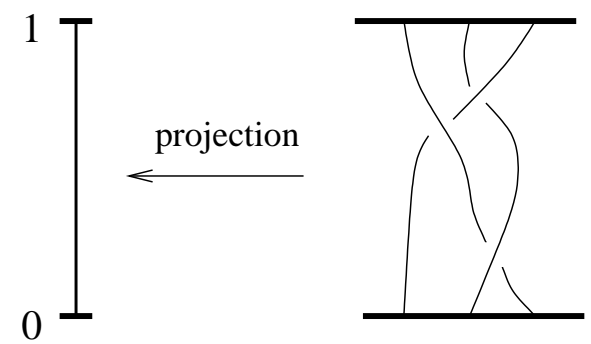

Thus, a braid is a embedding of a 1-manifold which is a disjoint union of closed intervals into $\mathbb{R}^{2} \times[0,1]$ so that the projection onto $[0,1]$ has no critical points, and the boundary of the 1-manifold is mapped to $2 n$ points

$$
(1,0,0),(2,0,0), \ldots,(n, 0,0),(1,0,1),(2,0,1), \ldots,(n, 0,1) \in \mathbb{R}^{2} \times[0,1] .
$$

The first $n$ points on this list belong to the bottom $\mathbb{R}^{2}$, i.e. to $\mathbb{R}^{2} \times\{0\}$, the last $n$ points to $\mathbb{R}^{2} \times\{1\}$.

A braid cobordism between braids $g, h \in \mathrm{Br}_{n}$ is a compact surface $S$ with boundary and corners, smoothly and properly embedded in $\mathbb{R}^{2} \times[0,1]^{2}$, such that: 


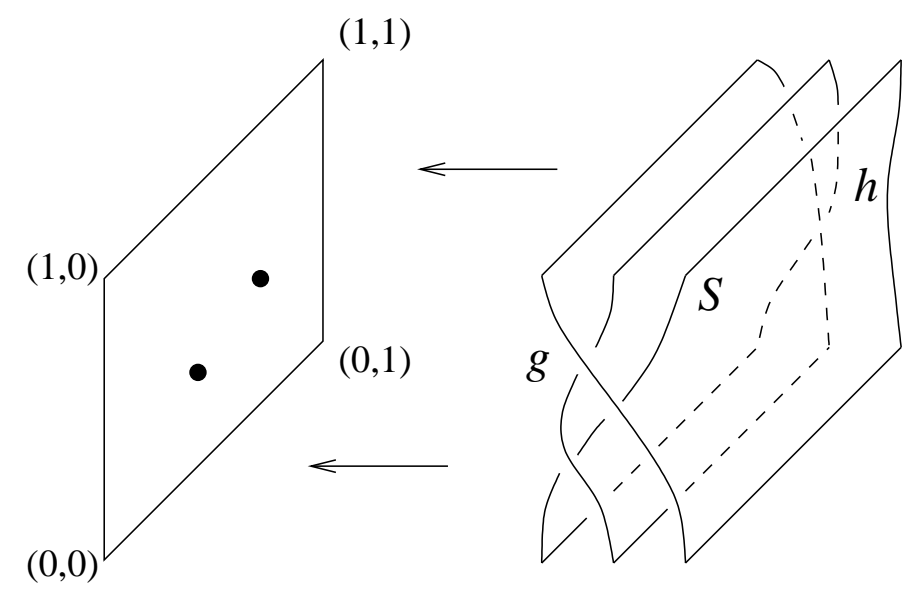

Figure 1: A depiction of a braid cobordism (mostly of its boundary), and its projection onto the unit square. Dots in the square are the simple branch points of the projection. The image of $g$ is the edge $[0,1] \times\{0\}$ of the square. The image of $h$ is the edge $[0,1] \times\{1\}$ of the square. Corners of $S$ are mapped to the vertices of the square.

- The boundary of $S$ is the union of four 1-manifolds

$$
\begin{aligned}
& S \cap\left(\mathbb{R}^{2} \times[0,1] \times\{0\}\right)=g, \\
& S \cap\left(\mathbb{R}^{2} \times[0,1] \times\{1\}\right)=h, \\
& S \cap\left(\mathbb{R}^{2} \times\{0\} \times[0,1]\right)=\{1,2, \ldots, n\} \times\{0\} \times[0,1], \\
& S \cap\left(\mathbb{R}^{2} \times\{1\} \times[0,1]\right)=\{1,2, \ldots, n\} \times\{1\} \times[0,1] .
\end{aligned}
$$

- The projection of $S$ onto $[0,1]^{2}$ is a branched covering with simple branch points only.

A braid cobordism is schematically depicted in Figure 1.

Braid cobordisms which are isotopic rel boundary via an isotopy through braid cobordisms are called equivalent. Let $\mathcal{B C}_{n}$ be the category with $n$-stranded braids as objects and isotopy classes (rel boundary) of braid cobordisms as morphisms. Composition of a morphism $S_{1}$ from $g$ to $h$ with a morphism $S_{2}$ from $h$ to $k$ is the braid cobordism $S_{2} S_{1}$ given by concatenating $S_{2}$ and $S_{1}$ along their common boundary $h$; see Figure 1 .

Note that in $\mathcal{B C}_{n}$ two isotopic braids are isomorphic, but not equal. The category $\mathcal{B C}_{n}$ is monoidal, since given a morphism $S_{1}$ from $g_{1}$ to $h_{1}$ and a morphism $S_{2}$ from $g_{2}$ to $h_{2}$, we can concatenate them to a morphism $S_{2} \circ S_{1}$ from $g_{2} g_{1}$ to $h_{2} h_{1}$. The concatenation is done by gluing the top portion of $\partial\left(S_{1}\right)$ to the bottom portion of $\partial\left(S_{2}\right)$. The monoidal structure is not strictly associative, since braids $g_{3}\left(g_{2} g_{1}\right)$ and $\left(g_{3} g_{2}\right) g_{1}$ are isotopic but not equal. 

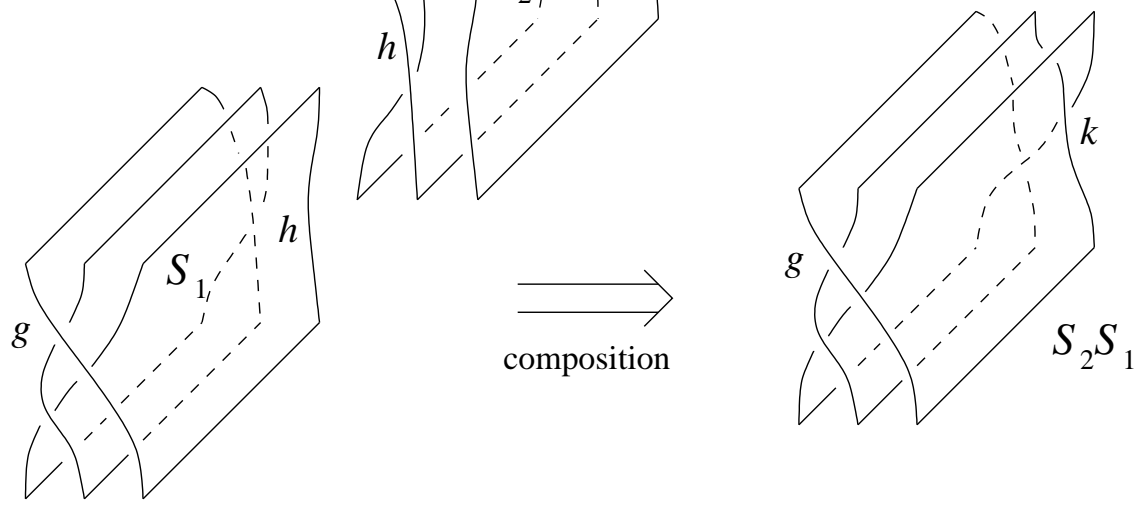

Figure 2: Composition of braid cobordisms.

In addition, the operation of placing braid cobordisms in parallel gives us a bifunctor

$$
\mathcal{B C}_{n} \times \mathcal{B C}_{m} \longrightarrow \mathcal{B C}_{n+m}
$$

Braid cobordisms are called simple braided surfaces in [Ka, Section 16], and originally appeared in Rudolph $[\mathbf{R u}]$. Braid cobordisms from the trivial braid to itself, with corners smoothed out, are called simple surface braids in the literature [Ka], [CS2, Section 3], and were introduced by O. Viro.

In Section 3.1 we recall a combinatorial version of $\mathcal{B C}_{n}$, following $[\mathbf{C S 1}],[\mathbf{C S 2}$, Section 3]. A braid cobordism can be described diagrammatically, via Carter and Saito's braid movies. This leads to a category, denoted $\mathcal{B C}_{n}^{c}$, with braid words as objects and braid movies, modulo braid movie moves, as morphisms. We call $\mathcal{B C}_{n}^{c}$ the category of combinatorial braid cobordisms. The categories $\mathcal{B C}_{n}$ and $\mathcal{B C}_{n}^{c}$ are equivalent.

In the beginning of Section 2 we define rings $A_{n}$, suitable triangulated categories $\mathcal{C}\left(A_{n}\right)$ of complexes of graded $A_{n}$-modules, and recall how to construct a weak braid group action on $\mathcal{C}\left(A_{n}\right)$. With every braid word $g$ we associate a functor $F_{g}$ such that $F_{g} \cong F_{h}$ if $g, h$ define the same braid in $\operatorname{Br}_{n+1}$. Let $\operatorname{Fun}\left(A_{n}\right)$ be the category with objects the exact endofunctors in $\mathcal{C}\left(A_{n}\right)$ and morphisms the natural transformations of functors.

Theorem 1.1. The braid group action on $\mathcal{C}\left(A_{n}\right)$ extends to a projective monoidal functor from the category $\mathcal{B C}_{n+1}^{c}$ of combinatorial braid cobordisms to the category $\operatorname{Fun}\left(A_{n}\right)$. 
To every braid cobordism $S$ between braid words $g$ and $h$ we assign a natural transformation $F_{S}: F_{g} \longrightarrow F_{h}$, well-defined up to overall minus sign. The presence of the minus sign explains why we use the word projective in the statement of the theorem. There are equalities of natural transformations

$$
F_{S_{2}} F_{S_{1}}= \pm F_{S_{2} S_{1}}
$$

for any pair of braid cobordisms $S_{1}$ from $g$ to $h$ and $S_{2}$ from $h$ to $k$.

The functor $F$ intertwines the monoidal structures of the two categories: $F_{g h}$ $\cong F_{g} \circ F_{h}$ and $F_{S_{2}} \circ F_{S_{1}}= \pm F_{S_{2} \circ S_{1}}$ for any cobordisms $S_{1}, S_{2}$ between $g_{1}, h_{1}$ and $g_{2}, h_{2}$, respectively.

Composing our functor with the equivalence $\mathcal{B C}_{n+1} \cong \mathcal{B C}_{n+1}^{c}$, we obtain a projective monoidal functor from the topological category $\mathcal{B C}_{n+1}$ of braid cobordisms to the algebraic category $\operatorname{Fun}\left(A_{n}\right)$ of natural transformations between exact functors in $\mathcal{C}\left(A_{n}\right)$.

This example serves as the first illustration of the principle:

Interesting braid group actions on triangulated categories extend to projective representations of the category of braid cobordisms.

In Sections 2 and 3 we list many additional examples when a braid group action on a triangulated category can be nontrivially extended to braid cobordisms. This multitude of examples motivates us to elevate the above principle to the definition of categorification of a braid group action. From here on by a categorification of a braid group representation we mean a projective functor from $\mathcal{B C}_{n}$ to the category of exact endofunctors in a triangulated category, as outlined above and defined in Definition 3.3 of Section 3.1 below.

Our requirement that the representation $F$ of braid cobordisms be only a projective functor is similar to considering weak braid group actions only, while requiring that $F$ be a functor should be analogous to considering genuine braid group actions. We expect that most, if not all, of the projective functors $\mathcal{B C}_{n} \stackrel{F}{\longrightarrow} \operatorname{Fun}(\mathcal{C})$ can be turned into genuine functors. The diagram below summarizes how the authors currently think about braid group actions on triangulated categories, each arrow denoting a structural upgrade.

\section{Projective representation of the braid cobordism category by exact functors and natural transformations}

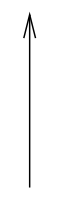

Weak braid group action on a triangulated category

\section{Representation of the category of braid cobordisms by exact functors and natural transformations}

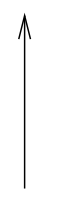

Genuine braid group action on a triangulated category 
At the end of Section 3.3 we sketch the relation between Rouquier's definition of a categorification of a braid group action and ours.

In the last two sections we turn to coherent sheaves, where the proofs become more technical but the ideas are essentially the same. In Section 4 we exhibit an action of $\mathrm{Br}_{n}$ on $D^{b}\left(T^{*} \mathrm{Fl}\right)$, the derived category of coherent sheaves on the cotangent bundle to the variety of full flags in $\mathbb{C}^{n}$. The functors we use are family versions of the Dehn twists about -2-curves of $[\mathbf{K S}, \mathbf{S T}]$, as considered in $[\mathbf{H o}, \mathbf{S z 1}]$. Locally, away from some closed subsets of $T^{*} F l$, the subschemes we twist about form a family $A_{n-1}$-chain in the sense of [Sz2], but, since they all contain the zero section $\mathrm{Fl} \subset T^{*} \mathrm{Fl}$, the proof of the braid relations is much more complicated than in $[\mathbf{S T}, \mathbf{S z 2}]$ (due to the extra term $U_{i j}$ in Proposition 4.10, for instance).

Given an $A_{n}$-chain of -2-curves in a surface, it is fairly easy to show that the structure sheaf of the whole chain (twisted by an appropriate line bundle) gives a spherical object whose Dehn twist extends the braid group action of $[\mathbf{S T}]$ to an affine braid group action [ST, Example 3.9]. While such a simple description does not generalise to $T^{*} F l$, one can show that the extra twist is given by a certain product of the original generators conjugated by tensoring by a line bundle. The resulting formula does generalise (23), so in Section 4.5 we extend our action to one of the affine braid groups on $D^{b}\left(T^{*} \mathrm{Fl}\right)$.

In Section 5.1 we extend the braid group action $[\mathbf{S T}]$ on the derived category of coherent sheaves of certain surfaces to a projective action of the braid cobordism category.

Theorem 1.2. Fix a smooth quasiprojective complex surface $X$ with an $A_{n}$-chain of -2-curves. Then the weak braid group action on $D^{b}(X)$ of $[\mathbf{S T}]$ extends to a projective action of $\mathcal{B C}_{n+1}^{c}$.

Finally in Section 5.2 we show how to do the same for $D^{b}\left(T^{*} F l\right)$. We expect these two generalisations of the braid group action on $D^{b}\left(T^{*} F l\right)$ to be compatible, giving a genuine action of the affine braid cobordism category.

Theorem 1.3. There is a projective action of $\mathcal{B C}_{n}^{c}$ on the derived category of coherent sheaves on the cotangent bundle of the full flag variety.

When the second author spoke about the braid group action on $D^{b}\left(T^{*} F l\right)$ in Leeds in November 2005, Raphaël Rouquier informed us of another way to produce a braid group action (which is hard to make explicit and so difficult to confirm is the same as ours). Start with the braid group action on the derived category of constructible sheaves on the flag variety (see for example [Ro1]). This is induced by kernels of constructible sheaves on the product. Their associated mixed Hodge modules (see for instance page 18 of [Ro2]) interpolate between (derived categories of) constructible sheaves and $\mathbb{C}^{*}$-equivariant coherent sheaves on the cotangent bundle. This gives $\mathbb{C}^{*}$-equivariant kernels generating a braid group action on the derived category of coherent sheaves on the cotangent bundle of the flag variety.

Recently Bezrukavnikov, Mirković and Rumynin [BMR] have also produced an affine braid group action (for any group, not just of type $A_{n}$ ) by very different and sophisticated finite characteristic methods which we cannot claim to understand. 
Assuming that their action, which also seems to be hard to make explicit, coincides with the explicit action of Section 4 then we show it extends to an action of the braid cobordism category.

\section{Acknowledgements}

M. K. would like to thank Tom Mrowka and Peter Ozsváth for the opportunity to lecture on some of the material in this paper at the IAS/Park City summer school 2006 and Scott Carter for enlightening email discussions. R. P. T. would like to thank Martin Bridson, Tom Bridgeland, Daniel Huybrechts, Ivan Smith, Simon Willerton and most especially Paul Seidel for valuable discussions. M. K. gratefully acknowledges NSF partial support via grant DMS-0407784. R. P. T. is partially supported by the Royal Society and the Leverhulme Trust.

\section{Examples of actions of braid groups and braid cobordisms on triangulated categories}

Example 2.1 (Spherical objects). Let $A_{n}$, for $n>2$, be the quotient of the path ring of the quiver

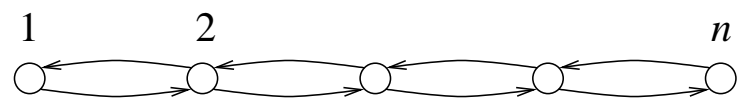

by the relations

$$
\begin{aligned}
(i|i+1| i+2) & =0, \quad 1 \leqslant i \leqslant n-2, \\
(i|i-1| i-2) & =0, \quad 3 \leqslant i \leqslant n, \\
(i|i-1| i) & =(i|i+1| i), \quad 2 \leqslant i \leqslant n-1 .
\end{aligned}
$$

We define $A_{2}$ as the quotient of the above quiver ring, for $n=2$, by the relations $(1|2| 1 \mid 2)=0=(2|1| 2 \mid 1)$. Let $A_{1}=\mathbb{Z}\left[X_{1}\right] /\left(X_{1}^{2}\right)$.

The ring $A_{n}$ is a free abelian group of rank $4 n-2$ and has a basis given by $(i), 1 \leqslant i \leqslant n$, which are length zero paths and minimal idempotents, all possible paths of length one (which are $(i \mid i \pm 1)$ ), and

$$
X_{i} \stackrel{\text { def }}{=}(i|i-1| i), \quad i \geqslant 2, \quad X_{1}=(1|2| 1) .
$$

Denote this basis by $B\left(A_{n}\right)$. The center of $A_{n}$ is a free abelian group with the basis

$$
1=\sum_{1 \leqslant i \leqslant n}(i), \quad X_{1}, \quad X_{2}, \quad \ldots, \quad X_{n}
$$

The ring $A_{n}$ is naturally graded by path length. Let

$$
P_{i} \stackrel{\text { def }}{=} A_{n}(i), \quad{ }_{i} P \stackrel{\text { def }}{=}(i) A_{n} .
$$


These are indecomposable left, respectively right, projective $A_{n}$-modules. Consider the complexes of $A_{n}$-bimodules

$$
\begin{array}{ll}
R_{i}: & 0 \longrightarrow P_{i} \otimes{ }_{i} P \stackrel{\beta_{i}}{\longrightarrow} A_{n} \longrightarrow 0 \\
R_{i}^{\prime}: & 0 \longrightarrow A_{n} \stackrel{\gamma_{i}}{\longrightarrow} P_{i} \otimes{ }_{i} P \longrightarrow 0
\end{array}
$$

with $A_{n}$ placed, in both complexes, in cohomological degree 0 . Here $\beta_{i}$ is the composition of the inclusion and multiplication

$$
P_{i} \otimes{ }_{i} P \subset A_{n} \otimes A_{n} \longrightarrow A_{n},
$$

while $\gamma_{i}$ is determined by

$$
\gamma_{i}(1)=\sum \alpha_{1} \otimes \alpha_{2}
$$

the sum being over all $\alpha_{1}, \alpha_{2} \in B\left(A_{n}\right)$ such that $\alpha_{2} \alpha_{1}=X_{i}$. Thus, if $1<i<n$,

$$
\begin{aligned}
\gamma_{i}(1)= & (i-1 \mid i) \otimes(i \mid i-1)+(i+1 \mid i) \otimes(i \mid i+1) \\
& +(i) \otimes(i|i+1| i)+(i|i+1| i) \otimes(i) .
\end{aligned}
$$

The projectives $P_{i},{ }_{i} P$ are examples of spherical objects, so-called since homologically they resemble spheres,

$$
\operatorname{End}\left(P_{i}\right) \cong \mathbb{Z}\left[X_{i}\right] /\left(X_{i}^{2}\right) \cong \mathrm{H}^{*}\left(\mathbb{S}^{k}, \mathbb{Z}\right)
$$

(where $k=2$ if we use the path length grading on $A_{n}$ ), and satisfy a duality property, which in this example manifests itself in $A_{n}$ being a symmetric ring.

Consider the category of complexes of $A_{n}$-bimodules up to chain homotopies (the homotopy category, for short). Recall from $[\mathbf{K S}]$ that the following isomorphisms hold in this category:

$$
\begin{aligned}
R_{i} \otimes R_{i}^{\prime} & \cong A_{n} \cong R_{i}^{\prime} \otimes R_{i} \\
R_{i} \otimes R_{i+1} \otimes R_{i} & \cong R_{i+1} \otimes R_{i} \otimes R_{i+1} \\
R_{i} \otimes R_{j} & \cong R_{j} \otimes R_{i}, \quad|i-j|>1 .
\end{aligned}
$$

The tensor products above are taken over $A_{n}$. Let $\mathcal{C}\left(A_{n}\right)$ be one of the triangulated categories formed out of complexes of left $A_{n}$-modules, such as the derived category $D^{b}\left(A_{n}-\bmod \right)$, the homotopy category of complexes of $A_{n}$-modules, etc. Tensoring $M \in \mathcal{C}\left(A_{n}\right)$ with the complexes $R_{i}, R_{i}^{\prime}$ produces complexes of $A_{n}$-modules

$$
R_{i} \otimes_{A_{n}} M, \quad R_{i}^{\prime} \otimes_{A_{n}} M \in \mathcal{C}\left(A_{n}\right)
$$

We denote the resulting endofunctors in $\mathcal{C}\left(A_{n}\right)$ by $\mathcal{R}_{i}, \mathcal{R}_{i}^{\prime}$ :

$$
\mathcal{R}_{i}(M) \stackrel{\text { def }}{=} R_{i} \otimes_{A_{n}} M, \quad \mathcal{R}_{i}^{\prime}(M) \stackrel{\text { def }}{=} R_{i}^{\prime} \otimes_{A_{n}} M .
$$

The earlier bimodule isomorphisms translate into functor isomorphisms

$$
\begin{aligned}
\mathcal{R}_{i} \circ \mathcal{R}_{i}^{\prime} & \cong \operatorname{Id} \cong \mathcal{R}_{i}^{\prime} \circ \mathcal{R}_{i}, \\
\mathcal{R}_{i} \circ \mathcal{R}_{i+1} \circ \mathcal{R}_{i} & \cong \mathcal{R}_{i+1} \circ \mathcal{R}_{i} \circ \mathcal{R}_{i+1}, \\
\mathcal{R}_{i} \circ \mathcal{R}_{j} & \cong \mathcal{R}_{j} \circ \mathcal{R}_{i}, \quad|i-j|>1,
\end{aligned}
$$

implying that the $(n+1)$-stranded braid group $\operatorname{Br}_{n+1}$ acts weakly on $\mathcal{C}\left(A_{n}\right)$. It is proved in $[\mathbf{K S}]$ that this action is faithful (faithfulness in the case $n=2$ was shown 
in $[\mathbf{R Z}])$. Theorem 1.1 states that this action extends to braid cobordisms.

Braid cobordisms are a subset of tangle cobordisms $[\mathbf{B L}]$. Tangle cobordisms constitute a 2-category $\mathbb{T}$, with nonnegative integers as objects, tangles as morphisms, and tangle cobordisms as 2-morphisms. There are several known projective 2 -functors from $\mathbb{T}$ to the 2-category of triangulated categories. In the latter triangulated categories are objects, exact functors are 1-morphisms and natural transformations are 2-morphisms. Any such 2-functor restricts to a projective functor from the category of $n$-stranded braid cobordisms to the category of functors and natural transformations in some triangulated category. Examples 2.2-2.6 below are of this nature.

Example 2.2 (The rings $H^{n}$ ). A certain family of rings $H^{n}$ was introduced in [Kh1] and used in $[\mathbf{K h} \mathbf{1}, \mathbf{K h 2}]$ to define a projective 2-functor from a suitable version of the category $\mathbb{T}$ of tangle cobordisms to the 2-category of exact functors (and natural transformations) between the categories $\mathcal{C}\left(H^{n}\right)$, over various $n \geqslant 0$. Here $\mathcal{C}\left(H^{n}\right)$ is defined similarly to $\mathcal{C}\left(A_{n}\right)$. For instance, $\mathcal{C}\left(H^{n}\right)$ could be the derived category $D^{b}\left(H^{n}-\bmod \right)$.

In this construction, there is a sign indeterminacy in the natural transformation associated with a tangle cobordism.

Restricting from tangles to braids and from tangle cobordisms to braid cobordisms yields a projective functor from $\mathcal{B C}_{2 n}$ to $\operatorname{Fun}\left(H^{n}\right)$, the category of exact functors in $\mathcal{C}\left(H^{n}\right)$ and natural transformations between them. To a $2 n$-stranded braid we assign a complex of $H^{n}$-bimodules; tensoring with this complex is an endofunctor in $\mathcal{C}\left(H^{n}\right)$. To a braid cobordism there is assigned a homomorphism between complexes of $H^{n}$-bimodules, inducing a natural transformation between the corresponding functors.

If we take $\mathcal{C}\left(H^{n}\right)$ to be the homotopy category of complexes of finitely-generated graded projective $H^{n}$-modules (the rings $H^{n}$ are graded), then its Grothendieck group, after tensoring with $\mathbb{C}$ over $\mathbb{Z}\left[q, q^{-1}\right]$, become isomorphic to the space of $U_{q}\left(\mathfrak{s l}_{2}\right)$-invariants in $V^{\otimes 2 n}$, the $2 n$-th tensor power of the defining representation of the quantum group $U_{q}\left(\mathfrak{s l}_{2}\right)$. Thus, we obtain a categorification, in the sense of Definition 3.3, of the braid group action on $\operatorname{Inv}\left(V^{\otimes 2 n}\right)$.

Example 2.3. Bar-Natan's invariant of tangle cobordisms $[\mathbf{B N}]$ can be restricted to braid cobordisms and viewed in the framework of triangulated categories which are similar to but more general than $\mathcal{C}\left(H^{n}\right)$. His construction also restricts to a categorification of the braid group action on $\operatorname{Inv}\left(V^{\otimes 2 n}\right)$.

Example 2.4. An invariant of tangle cobordisms via highest weight categories was constructed by Stroppel [St1, St2], following conjectures in [BFK]. Restricting her invariant to braids and braid cobordisms results in a projective functor from $\mathcal{B C}_{n}$ to Fun $\left(D^{b}\left(\mathcal{O}^{n}\right)\right)$, the category of exact endofunctors in the derived category of $\mathcal{O}^{n}$, the latter a direct sum of certain parabolic subcategories of a regular block of the highest weight category for $\mathfrak{s l}_{n}$. In this example the projectivity (indeterminacy) takes values in $\mathbb{C}^{*}$, due to $\mathbb{C}$ being the ground field. The Grothendieck group of (the 
graded version of) $\mathcal{O}^{n}$ is isomorphic to $V^{\otimes n}$, and the projective functor

$$
\mathcal{B C}_{n} \longrightarrow \operatorname{Fun}\left(D^{b}\left(\mathcal{O}^{n}\right)\right)
$$

is a categorification of the braid group action on $V^{\otimes n}$.

Example 2.5. A categorification $[\mathbf{K h} 4]$ of the quantum $\mathfrak{s l}_{3}$ link invariant was extended to tangles and tangle cobordisms by MacKaay and Vaz $[\mathbf{M V}]$. After restricting their structure to braid cobordisms one should obtain a categorification of the action of the $3 n$-stranded braid group on $\operatorname{Inv}\left(V^{\otimes 3 n}\right)$, the $3 n$-th tensor power of the fundamental representation $V$ of quantum $\mathfrak{s l}_{3}$. More generally, their construction conjecturally categorifies spaces of invariants in arbitrary tensor products of $V$ and its dual $V^{*}$. The braid group $\operatorname{Br}_{n}$ acts on $V^{\otimes n}$, and hence on $\operatorname{Inv}\left(V^{\otimes n} \otimes W\right)$, where $W$ is any tensor product of $V$ 's and $V^{*}$ 's. Following [MV], one should also obtain a categorification of the braid group action on $\operatorname{Inv}\left(V^{\otimes n} \otimes W\right)$, by adding dummy strands. In this example, the projectivity is only in the minus sign.

Example 2.6. Yet another invariant of tangle cobordisms was introduced in $[\mathbf{K R}]$, based on matrix factorizations. A matrix factorization of $f \in R$, where $R$ is a polynomial algebra, consists of a pair of free $R$-modules $M^{0}, M^{1}$ and $R$-module maps

$$
M^{0} \stackrel{d}{\longrightarrow} M^{1} \stackrel{d}{\longrightarrow} M^{0}
$$

such that $d^{2}(m)=f m$ for any $m \in M^{0}, M^{1}$. The category of matrix factorizations up to chain homotopy is triangulated. Given a braid word $g$, the construction of $[\mathbf{K R}]$ associates to it a complex of matrix factorizations in $2 n$ variables $x_{1}, \ldots, x_{n}, y_{1}, \ldots, y_{n}$ with

$$
f=\sum_{i=1}^{n} x_{i}^{m+1}-y_{i}^{m+1},
$$

where $n$ is the number of strands. The isomorphism class of this complex in the category of complexes of matrix factorizations up to chain homotopy is an invariant of the braid. A braid cobordism between braids $g, h$ induces a homomorphism of corresponding complexes, well-defined up to rescaling by nonzero rational numbers. One can easily restate this construction in the language of functors, resulting in yet another categorification of a braid group action subject to the constraints of Definition 3.3. We have no clue what representation of the braid group this categorification categorifies. It should contain a subrepresentation isomorphic to $\operatorname{Inv}\left(V^{\otimes n} \otimes\left(V^{*}\right)^{\otimes n}\right)$, where $V$ is the defining representation of quantum $\mathfrak{s l}_{m}$ and the braid group acts on the first $n$ factors.

Example 2.7 (Highest weight categories and flag varieties). We start with the triangular decomposition $\mathfrak{s l}_{n}=\mathfrak{n}_{+} \oplus \mathfrak{h} \oplus \mathfrak{n}_{-}$of the Lie algebra $\mathfrak{s l}_{n}$, where $\mathfrak{n}_{+}$, respectively $\mathfrak{n}_{-}$, are the Lie algebras of strictly upper-triangular, respectively lower-triangular, matrices, and $\mathfrak{h}$ the Lie algebra of traceless diagonal matrices. The category $\mathcal{O}$ is a full subcategory of the category of finitely-generated $\mathfrak{s l}_{n}$-modules. A finitelygenerated $\mathfrak{s l}_{n}$-module $M$ belongs to the highest weight category $\mathcal{O}$ if and only if 
$\mathfrak{h}$ acts semisimply and $\mathfrak{n}_{+}$locally-nilpotently on $M$; see $[\mathbf{B G}]$. The trivial onedimensional $\mathfrak{s l}_{n}$ representation defines a central character $\chi: Z \longrightarrow \mathbb{C}$, a homomorphism from the center of $U\left(\mathfrak{s l}_{n}\right)$ to $\mathbb{C}$. Define $\mathcal{O}_{0}$, the trivial central character block of $\mathcal{O}$, as the full subcategory of $\mathcal{O}$ consisting of modules annihilated by some power of $\operatorname{ker}(\chi) \subset Z$. This category is equivalent to the category of finite-dimensional modules over some finite-dimensional Koszul $\mathbb{C}$-algebra $B_{n}$, although a simple description of $B_{n}$ is not known even for $n=5$.

The category $\mathcal{O}_{0}$ has $n$ ! isomorphism classes of simple modules. There are exact self-adjoint endofunctors $\theta_{i}$ in $\mathcal{O}_{0}$ (called translation across the $i$-th wall, $1 \leqslant i$ $\leqslant n-1)$ which come with natural transformations $\theta_{i} \longrightarrow \operatorname{Id}$ and $\operatorname{Id} \longrightarrow \theta_{i}$. Let $\mathcal{C}\left(\mathcal{O}_{0}\right)$ be either the derived category of $\mathcal{O}_{0}$ or the category of complexes of objects in $\mathcal{O}_{0}$ up to chain homotopies. Taking an object $M \in \mathcal{C}\left(\mathcal{O}_{0}\right)$ to the total complex of

$$
0 \longrightarrow \theta_{i} M \longrightarrow M \longrightarrow 0
$$

is an invertible functor $\mathcal{R}_{i}$ in $\mathcal{C}\left(\mathcal{O}_{0}\right)$, with the inverse functor $\mathcal{R}_{i}^{\prime}$ taking $M$ to the total complex of

$$
0 \longrightarrow M \longrightarrow \theta_{i} M \longrightarrow 0
$$

It follows from [MS1], [MS2, Proposition 10.1] that the functors $\mathcal{R}_{i}$ satisfy the braid relations $(6)-(8)$ and define a weak braid group action on $\mathcal{C}\left(\mathcal{O}_{0}\right)$.

A similar braid group action in the Koszul dual case of Zuckerman functors (rather than translation functors) acting on $D^{b}\left(\mathcal{O}_{0}\right)$ was given a detailed treatment by Rouquier [Ro1, Ro3], who showed, in particular, that the action is genuine. The derived category $D^{b}\left(\mathcal{O}_{0}\right)$ embeds into the derived category $D^{b}(Y)$ of sheaves of vector spaces on the variety $Y$ of full flags in $\mathbb{C}^{n}$. For each $i=1, \ldots, n-1$ there exists a correspondence $Y_{i} \subset Y \times Y$ which consists of pairs of flags that coincide except at the $i$-th term. The convolution with the constant sheaf on $Y_{i}$ extended by 0 to the entire $Y \times Y$ is an invertible functor $\mathcal{R}_{i}$ in $D^{b}(Y)$. These functors generate a genuine action of the braid group on $D^{b}(Y)$; see [Ro1].

This construction admits a variation where, for each decomposition $\mu=$ $\left(\mu_{1}, \ldots, \mu_{k}\right)$ of $n$, we consider the variety $Y_{\mu}$ of partial flags in $\mathbb{C}^{n}$, of dimensions $\mu_{1}, \mu_{1}+\mu_{2}, \ldots, n-\mu_{k}$, and construct an invertible functor from $D^{b}\left(Y_{\mu}\right)$ to $D^{b}\left(Y_{s_{i} \mu}\right)$, where $s_{i} \mu$ is the decomposition given by transposing $\mu_{i}$ and $\mu_{i+1}$. The functor is given by convolution with $Y_{i} \subset Y_{\mu} \times Y_{s_{i} \mu}$, where the latter consists of pairs of flags that coincide except at the $i$-th term, while the intersection of the $i$-th terms is the smallest possible. These functors, which appeared in $[\mathbf{K h} \mathbf{3}$, Proposition 7], satisfy braid relations, and the same argument as in Rouquier [Ro1] implies that the braid group action on the derived category of sheaves on the disjoint union of $Y_{s \mu}$, over all elements $s$ of the symmetric group $S_{k+1}$, is genuine. For generic $\mu$, this action can only be extended trivially to braid cobordisms with branch points, due to the absence of nonzero natural transformations between $F_{1}$ and $F_{\sigma_{i}}$.

A closely related example of braid group categorification via Rouquier complexes will be discussed below in Section 3.3. 
Homology, Homotopy and Applications, vol. 9(2), 2007
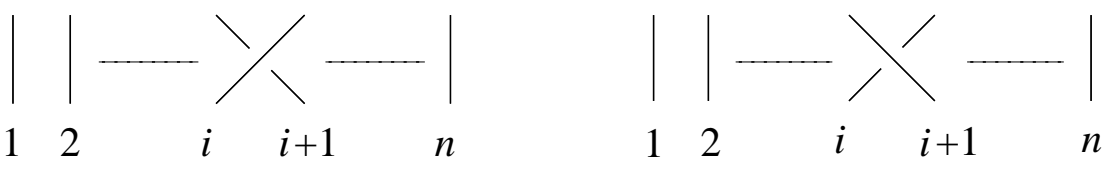

Figure 3: On the left: braid $\sigma_{i}$. On the right: braid $\sigma_{i}^{-1}$.

\section{Invariants of braid cobordisms}

\subsection{Braid cobordisms and braid group categorifications}

Combinatorial braid cobordisms. The category $\mathcal{B C}_{n}$ of $n$-stranded braid cobordisms admits a combinatorial version which we denote $\mathcal{B C}_{n}^{c}$. Its objects are $n$ stranded braid words, i.e. arbitrary finite sequences of generators $\sigma_{i}, 1 \leqslant i \leqslant n-1$, and their inverses $\sigma_{i}^{-1}$; see Figure 3 .

A morphism between braid words is a finite sequence of transformations of two types:

I. Reidemeister moves of braid words, including

$$
\begin{aligned}
\tau_{1} \sigma_{i} \sigma_{i}^{-1} \tau_{2} & \leftrightarrow \tau_{1} \tau_{2}, \\
\tau_{1} \sigma_{i} \sigma_{j} \tau_{2} & \leftrightarrow \tau_{1} \sigma_{j} \sigma_{i} \tau_{2}, \quad|i-j|>1, \\
\tau_{1} \sigma_{i} \sigma_{i+1} \sigma_{i} \tau_{2} & \leftrightarrow \tau_{1} \sigma_{i+1} \sigma_{i} \sigma_{i+1} \tau_{2} .
\end{aligned}
$$

$\tau_{1}$ and $\tau_{2}$ are arbitrary braid words. The empty word is allowed. Each move has several versions, and we will not write all of them, instead referring the reader to [CS2, Section 3] for details. For instance, in the second move above we could have substituted $\sigma_{i}^{-1}$ for $\sigma_{i}$, and, independently, $\sigma_{j}^{-1}$ for $\sigma_{j}$.

II. Addition or removal of a single $\sigma_{i}$ or $\sigma_{i}^{-1}$ to or from a word:

$$
\tau_{1} \tau_{2} \leftrightarrow \tau_{1} \sigma_{i}^{ \pm 1} \tau_{2}
$$

A braid movie is a finite sequence of transformations of types I and II. Morphisms in the category $\mathcal{B C}_{n}^{c}$ are braid movies modulo equivalence relations of two types: movie moves, shown in Figures 4-6, and locality moves. The first ten movie moves are equations on sequences of Reidemeister moves of braids, while each sequence in moves 11 through 14 contains a unique type II transformation. A type II transformation is indicated by placing two little black triangles where a pair of adjacent vertical lines turns into $\sigma_{i}^{ \pm 1}$. In the figures, movies run from top to bottom, and represent a morphism in $\mathcal{B C}_{n}^{c}$; moves of these movies run left to right, representing equivalences between morphisms.

Each movie move has several variants, given by changing overcrossings to undercrossings and vice versa, whenever possible; reading each of the two sequences from bottom to top rather than from top to bottom (the latter is our default); reflecting all diagrams in a movie move about a vertical axis (if this results in a different version of the move), etc. Again, we refer to Carter and Saito [CS2, Section3] for complete explanations. 

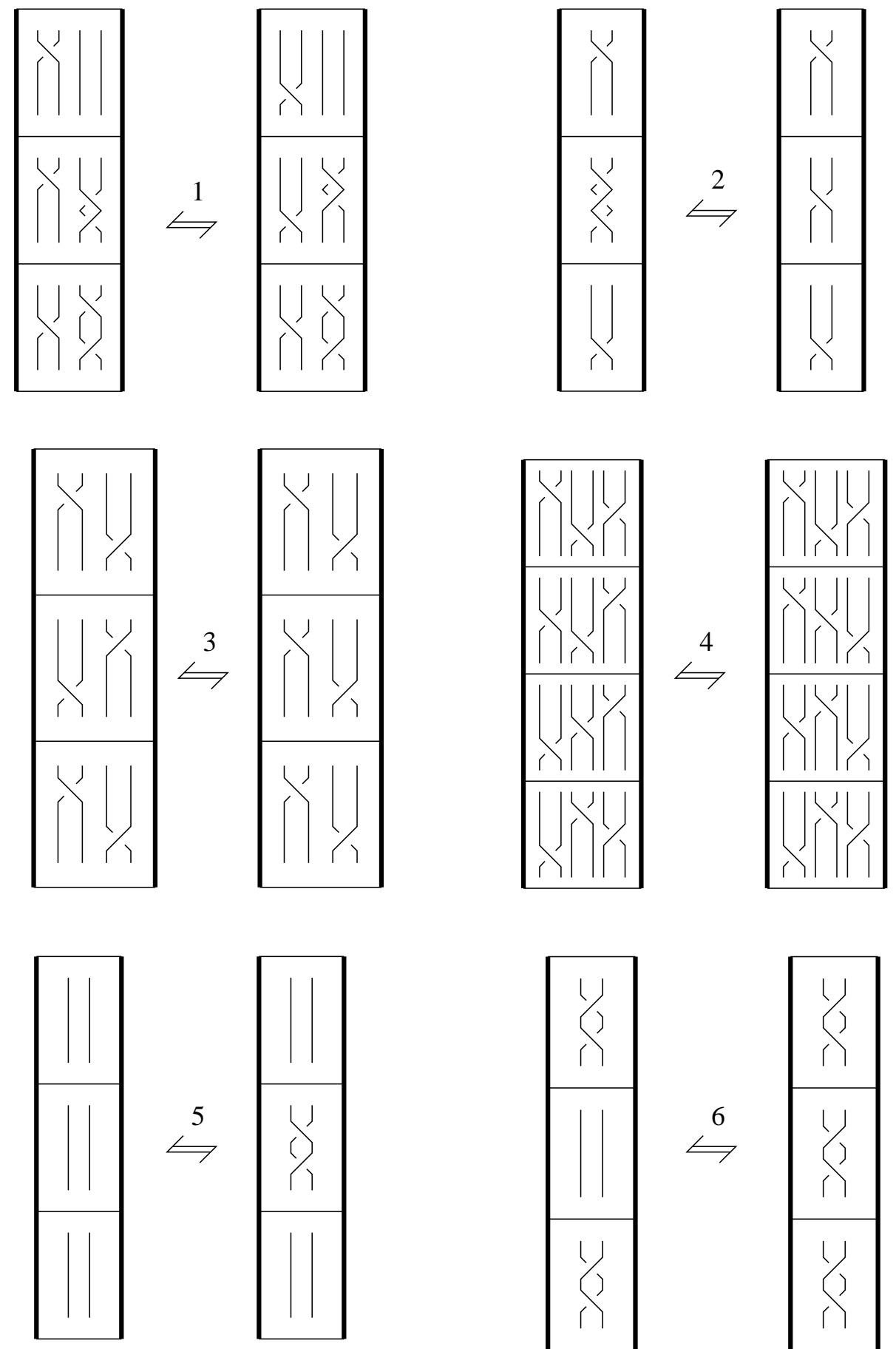

Figure 4: Carter and Saito's braid movie moves 1-6. 

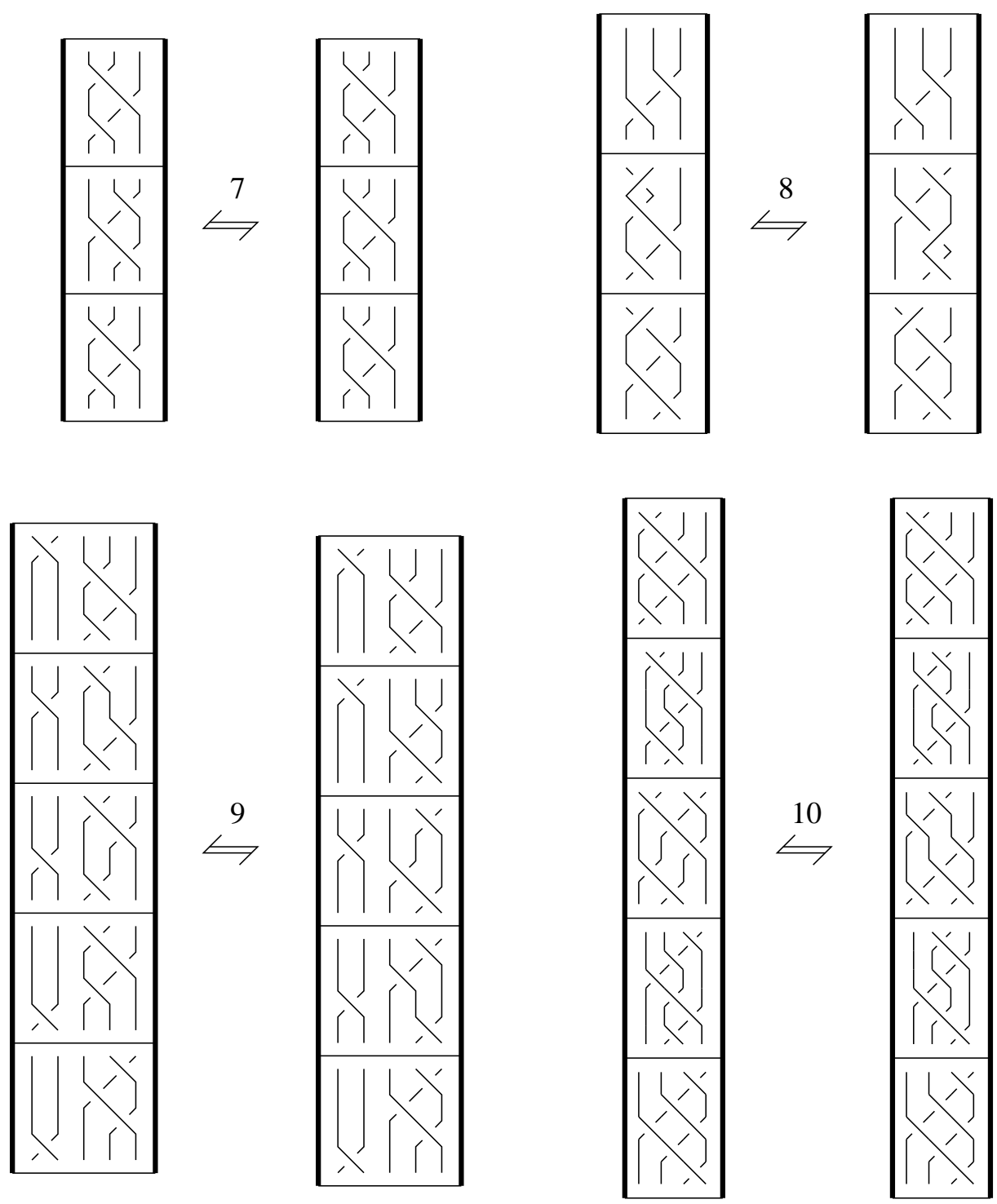

Figure 5: Braid movie moves 7-10. 

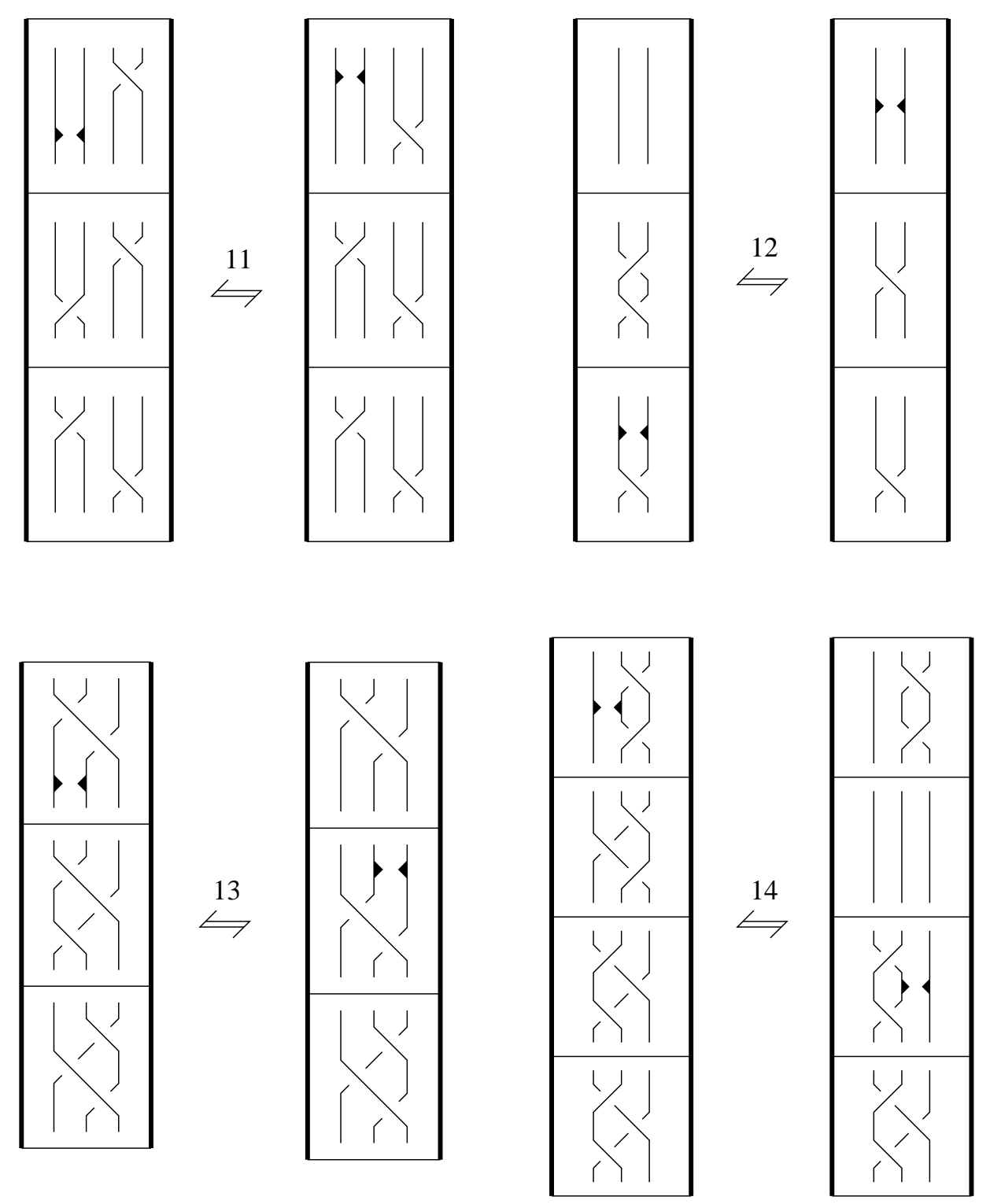

Figure 6: Braid movie moves 11-14. 

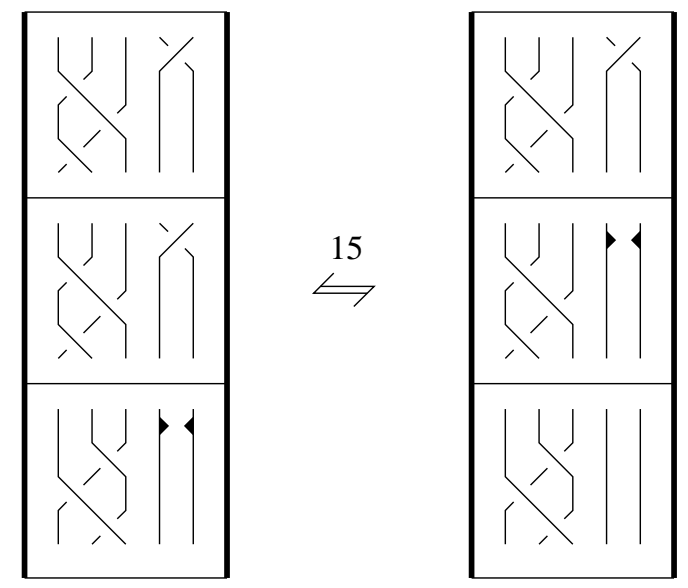

Figure 7: A locality movie move.

Locality moves are

$$
\left(\tau_{1} \tau_{2}, \tau_{1}^{\prime} \tau_{2}, \tau_{1}^{\prime} \tau_{2}^{\prime}\right)=\left(\tau_{1} \tau_{2}, \tau_{1} \tau_{2}^{\prime}, \tau_{1}^{\prime} \tau_{2}^{\prime}\right)
$$

where each of the pairs $\tau_{1}, \tau_{1}^{\prime}$ and $\tau_{2}, \tau_{2}^{\prime}$ are related by a single braid move, of either type. An example is depicted in Figure 7 , where $\tau_{1}=1$, the trivial braid word, $\tau_{1}^{\prime}=\sigma_{i+3}^{1}, \tau_{2}=\sigma_{i} \sigma_{i+1} \sigma_{i}$, and $\tau_{2}^{\prime}=\sigma_{i+1} \sigma_{i} \sigma_{i+1}$. We will refer to locality moves as braid movie moves of type 15 .

Morphisms between braid words $w_{1}$ and $w_{2}$ are finite sequences of braid words related by type I and II transformations modulo the equivalence relation generated by braid movie moves and locality relations.

To explain the meaning of type II transformations, we describe a functor from $\mathcal{B C}_{n}^{c}$ to $\mathcal{B C}_{n}$, the category of braid cobordisms. To each braid word we assign a braid in the usual way. To type I transformations we assign braid cobordisms given by isotopies of braids. Type II transformations correspond to elementary braid cobordisms which contain a single simple branch point when projected onto $[0,1]^{2}$. Our cryptic way of depicting type II transformations is deciphered in Figure 8. These cobordisms embed only in four dimensions rather than three - only after applying the twist on the right-hand side of Figure 8 in the fourth dimension can we embed the rest of the cobordism into three dimensions as the standard simple cobordism between a 1-manifold and its surgery (the second and third frames of the last movie in Figure 8) provided by a Morse function on a surface with an index one critical point (saddle point).

Since each braid movie move corresponds to a braid cobordism the above construction is a functor from $\mathcal{B C}_{n}^{c}$, the category of combinatorial braid cobordisms, to $\mathcal{B C}_{n}$, the category of braid cobordisms.

Proposition 3.1. This functor is an equivalence of categories: $\mathcal{B C}_{n}^{c} \cong \mathcal{B C}_{n}$.

Proof. See $[$ CS2] for a proof. 


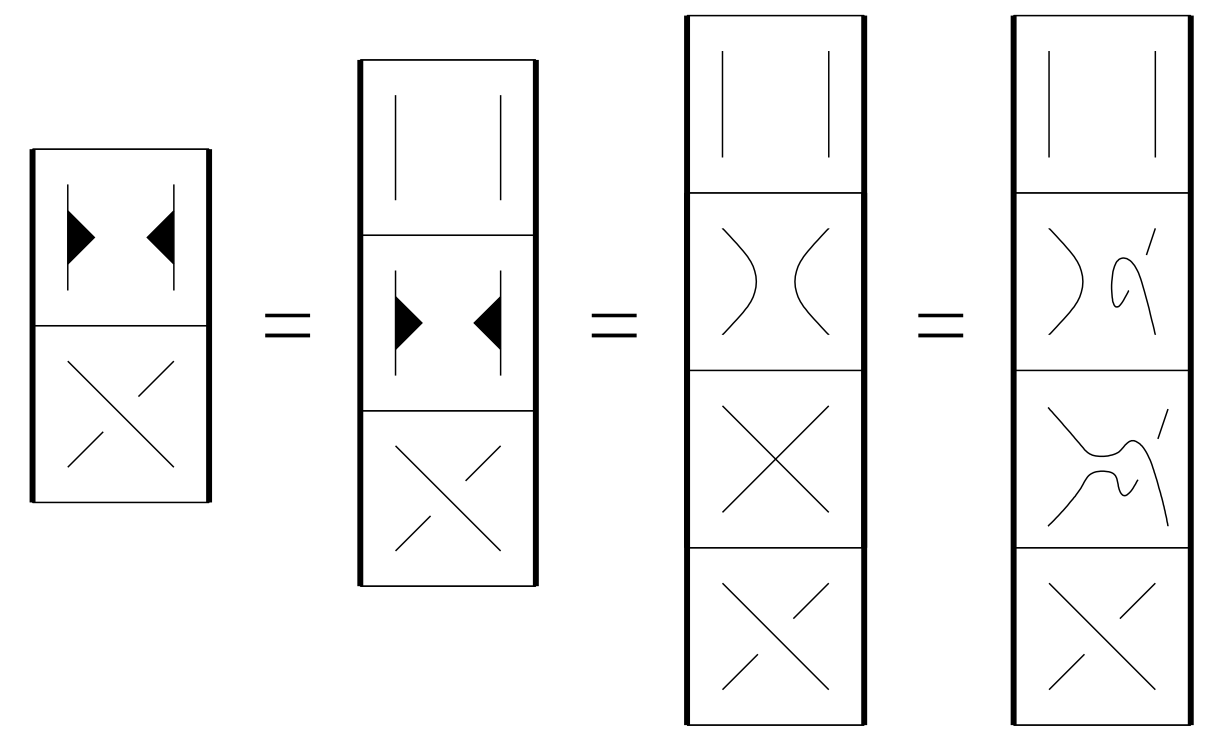

Figure 8: A type II braid transformation.

Polarization of braid cobordisms. Recall that a braid is positive if it can be represented as a product of $\sigma_{i}$, for $1 \leqslant i \leqslant n-1$. Likewise, a braid is called negative if it can be written as a product of $\sigma_{i}^{-1}$, for $1 \leqslant i \leqslant n-1$. For braid cobordisms there also exists an analogue of positivity. We say that a simple branch point of a braid cobordism is positive if, when represented combinatorially via a type II move, it increases braid positivity. Namely, positive type II moves are

$$
\begin{aligned}
\tau_{1} \tau_{2} & \longrightarrow \tau_{1} \sigma_{i} \tau_{2}, \\
\tau_{1} \sigma_{i}^{-1} \tau_{2} & \longrightarrow \tau_{1} \tau_{2}
\end{aligned}
$$

Negative type II moves are

$$
\begin{aligned}
\tau_{1} \tau_{2} & \longrightarrow \tau_{1} \sigma_{i}^{-1} \tau_{2}, \\
\tau_{1} \sigma_{i} \tau_{2} & \longrightarrow \tau_{1} \tau_{2} .
\end{aligned}
$$

Each type II move is thus either positive or negative. Notice that type II moves appear only in braid movie moves 11-15, and the property of being positive or negative is preserved by these movie moves.

Given a morphism $S$ in $\mathcal{B C}_{n}^{c}$, the numbers $p_{+}(S)$, respectively $p_{-}(S)$, of positive, respectively negative, type II transformations are invariants of $S$. Likewise, given a morphism $S$ in $\mathcal{B C}_{n}$, the numbers $p_{+}(S)$, respectively $p_{-}(S)$, of positive, respectively negative, double branch points are invariants of $S$. A braid cobordism is a braid isotopy if and only if $p_{+}(S)=p_{-}(S)=0$. We say that a morphism $S$ in $\mathcal{B C}_{n}^{c}$ or $\mathcal{B C}_{n}$ is positive if $p_{-}(S)=0$ and negative if $p_{+}(S)=0$. Positive braid cobordisms constitute a subcategory in $\mathcal{B C}_{n}$ (and the same for negative cobordisms). 

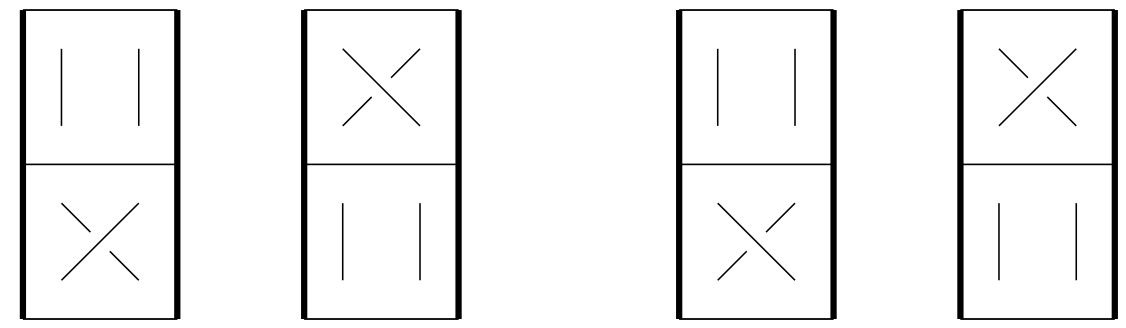

Figure 9: On the left: two movies of a positive branch point. On the right: two movies of a negative branch point.
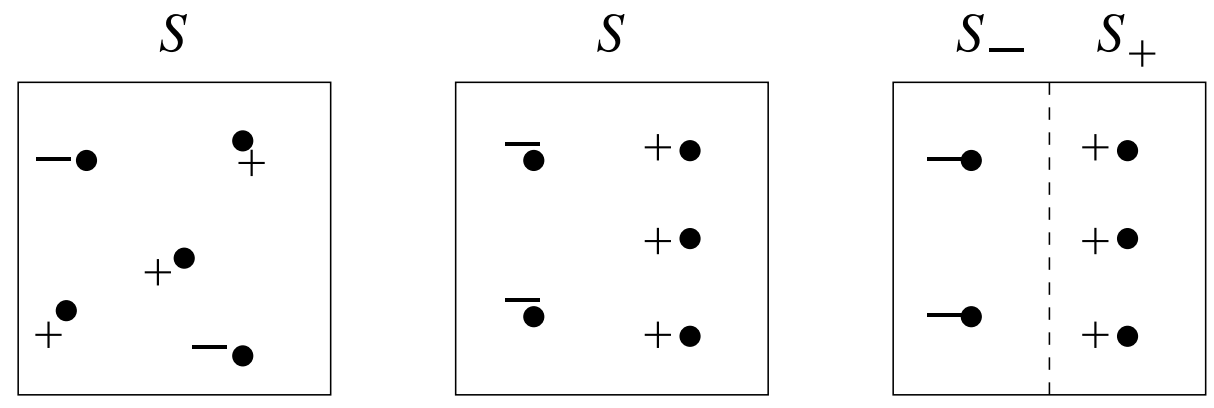

Figure 10: Moving branch points.

Positive braid movies constitute a subcategory in $\mathcal{B C}_{n}^{c}$ (and the same for negative braid movies). Our equivalence of categories $\mathcal{B C}_{n}^{c} \cong \mathcal{B C}_{n}$ takes positive, respectively negative, braid movies to positive, respectively negative, braid cobordisms.

Proposition 3.2. Any braid cobordism can be written, in more than one way, as a composition of a positive and a negative braid cobordism (in either order).

Proof. Project a braid cobordism $S$ onto the unit square, as in Figure 1. Next, isotop branch points so that the negative branch points are to the left of the positive ones; see Figure 10, and lift this isotopy to an isotopy of $S$. We can now write $S$ as the composition of two cobordisms, $S=S_{+} S_{-}$, with $S_{+}$positive and $S_{-}$negative (this decomposition is not unique even when $S$ has no double points). Likewise, we can write $S$ as $S_{-}^{\prime} S_{+}^{\prime}$ where $S_{-}^{\prime}$ is negative and $S_{+}^{\prime}$ is positive.

A braid cobordism is positive if and only if it can be realized, à la Rudolph $[\mathbf{R u}]$, as a piece of an algebraic curve in $\mathbb{C}^{2}$. The local model of a simple positive cobordism is the algebraic curve

$$
z_{2}^{2}=z_{1}
$$

in $\mathbb{C}^{2} \cong \mathbb{R}^{4}$ with its projection to $\left(x_{1}, y_{1}\right)=\left(\operatorname{Re} z_{1}, \operatorname{Im} z_{1}\right)$. For any fixed $\left(x_{1}, y_{1}\right)$ the $z_{2}$ coordinate gives two points in $\mathbb{C}$ which wind round each other as $x_{1}$ varies, giving 
a braid for $y_{1} \neq 0$. When $y_{1}=0$ the two strands cross. This shows the essential fourdimensionality of positive type II transformations. Similarly the simple negative type II transformation has local model $z_{2}^{2}=\bar{z}_{1}$.

\section{Categorification of the braid group.}

Definition 3.3. A categorification of a complex representation $V$ of the braid group $\mathrm{Br}_{n}$ is a (projective) monoidal functor $F$ from $\mathcal{B C}_{n}$ to the category of exact functors Fun $(\mathcal{C})$ in a triangulated category $\mathcal{C}$, and an isomorphism $V \cong K(\mathcal{C}) \otimes \mathbb{C}$ of braid group representations.

Thus, to each braid $g$ there is assigned an exact endofunctor $F_{g}$ in $\mathcal{C}$ and to each braid cobordism $S$ from $g$ to $h$ a natural transformation $F_{S}: F_{g} \longrightarrow F_{h}$, well-defined up to rescaling by invertible elements of the ground ring of $\mathcal{C}$. These must satisfy consistency conditions. The linear operator $\left[F_{g}\right]$ on $K(\mathcal{C}) \otimes \mathbb{C}$ must coincide with the action of $g$ on $V$ under the above isomorphism of these two complex vector spaces.

A genuine action of a braid group on $\mathcal{C}$ extends in a trivial way to a categorification in our sense, by making $F_{S}=0$ whenever $S$ has a double point and defining $V$ to be $K(\mathcal{C}) \otimes \mathbb{C}$. Likewise, given a categorification as above, we can modify it, by setting $F_{S}=0$ if, say, $S$ has a negative branch point, and otherwise doing nothing to $F_{S}$. More generally, given a categorification and two elements $\lambda_{+}, \lambda_{-}$of the ground ring of $\mathcal{C}$, we can rescale $F_{S}$ by $\lambda_{+}^{p_{+}(S)} \lambda_{-}^{p_{-}(S)}$ to get another categorification.

Naturally, we would like our categorifications to carry nontrivial information about braid cobordisms, and, whenever possible, we will avoid trivial or semi-trivial categorifications. By a semi-trivial categorification of a braid group representation we mean one with $F_{S}=0$ for all $S$ with a negative branch point, or for all $S$ with a positive branch point.

\subsection{Categorification from the rings $A_{n}$}

We now construct a categorification of the reduced Burau representation via the rings $A_{n}$. The braid group action on the homotopy category $\mathcal{C}\left(A_{n}\right)$, described in the previous section, lifts to the action on the homotopy category of complexes of graded $A_{n}$-modules. Indeed, $A_{n}$ has the natural grading by path length. The bimodules $A_{n}$ and $P_{i} \otimes{ }_{i} P$ are graded, and the bimodule maps $\beta_{i}$ and $\gamma_{i}$ have degrees 0 and 2 respectively. Hence, we can upgrade $R_{i}$ and $R_{i}^{\prime}$ to complexes of graded bimodules, shifting the grading of $P_{i} \otimes{ }_{i} P$ in $R_{i}^{\prime}$ down by 2 to make the differential preserve the grading:

$$
R_{i}^{\prime}: \quad 0 \longrightarrow A_{n} \stackrel{\gamma_{i}}{\longrightarrow} P_{i} \otimes{ }_{i} P\{-2\} \longrightarrow 0 .
$$

Tensoring with $R_{i}$ and $R_{i}^{\prime}$ induces a braid group action on the category of complexes of graded $A_{n}$-modules up to chain homotopy. We restrict to bounded complexes and denote the resulting category also by $\mathcal{C}\left(A_{n}\right)$. It is easy to see that on the Grothendieck group the braid group action descends to the reduced Burau representation of the braid group.

To extend to braid cobordisms, we assign a natural transformation of functors to each morphism in $\mathcal{B C}_{n+1}$, i.e. to each braid movie. This transformation will be 
defined up to an overall minus sign, resulting in a projective functor from $\mathcal{B C}_{n+1}$ to $\operatorname{Fun}\left(\mathcal{C}\left(A_{n}\right)\right)$. The functors associated to braid words are given by tensor products with complexes of graded bimodules. The latter complexes are tensor products of the complexes $R_{i}$ and $R_{i}^{\prime}$. Denote by $R(\sigma)$ the complex of graded bimodules associated with the braid word $\sigma$, and by $F_{\sigma}$ the functor of tensoring with $R(\sigma)$.

A homomorphism of complexes of bimodules induces a natural transformation of the corresponding functors, and our natural transformations will be of this form.

To a Reidemeister move of braid words we associate an isomorphism in the homotopy category of complexes of graded $A_{n}$-bimodules, namely, isomorphisms (3), (4), (5). Each of these isomorphisms is unique up to an overall minus sign. Indeed, suppose we are given two invertible complexes of graded bimodules over a graded ring $A$ and an isomorphism $u$ between these complexes. Then any isomorphism between these complexes has the form $m(z) u$ where $m$ is the multiplication by a degree 0 invertible element of the center of $A$ (see [Kh2, Section 3], for instance). The degree 0 part of $A_{n}$ is just $\mathbb{Z}$, its invertible elements are 1 and -1 , and the above claim follows.

To type II transformations we associate the following homomorphisms of complexes of bimodules.

The homomorphism $w_{1}$ from $R(\emptyset)$ to $R\left(\sigma_{i}\right)=R_{i}$ :

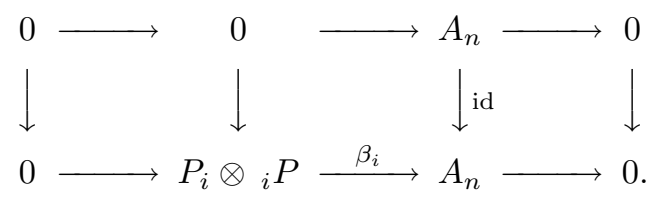

Here $R(\emptyset) \cong A_{n}$ is the identity bimodule complex, associated with the empty braid word $\emptyset$, corresponding to the trivial braid.

The homomorphism $w_{2}$ from $R\left(\sigma_{i}^{-1}\right)$ to $R(\emptyset)$ :

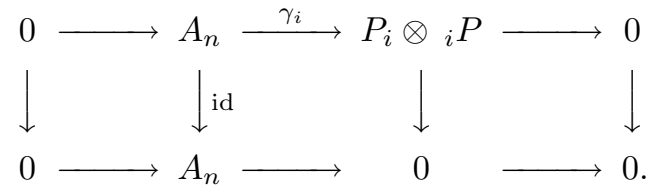

Notice that the above two homomorphisms are assigned to elementary movies of positive branch points. In both cases, the map is the identity on $A_{n}$.

The homomorphism $w_{3}$ from $R\left(\sigma_{i}\right)$ to $R(\emptyset)$ :

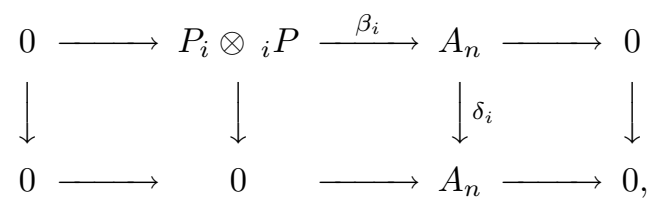

where $\delta_{i}(1)=X_{i-1}-X_{i+1}$. This condition determines the bimodule homomorphism $\delta_{i}$ uniquely. Notice that $\delta_{i} \beta_{i}=0$. 
The homomorphism $w_{4}$ from $R(\emptyset)$ to $R\left(\sigma_{i}^{-1}\right)$ :

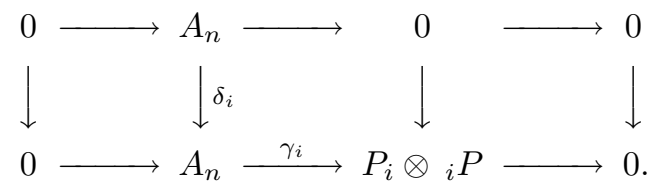

Thus, to the four braid movies in Figure 9 we assign the homomorphisms $w_{1}$, $w_{2}, w_{3}, w_{4}$ correspondingly (from left to right).

Given a braid movie $S$ from braid word $g$ to braid word $h$ we assign to $S$ the homomorphism $R(S): R(g) \longrightarrow R(h)$ of complexes of graded bimodules, the latter a composition of homomorphisms, described above, corresponding to elementary braid movies. This homomorphism preserves cohomological degree of complexes, and has internal degree $2 p_{-}(S)$, since the bimodule homomorphism $\delta_{i}$, corresponding to a movie of a negative branch point, has degree 2 .

Proof of Theorem 1.1. We show that the above assignment extends to a projective functor from $\mathcal{B C}_{n+1}$ to $\operatorname{Fun}\left(\mathcal{C}\left(A_{n}\right)\right)$. This is equivalent to checking that the assignment is compatible with the braid movie moves 1-15. Each braid movie move contains two movies $S_{1}, S_{2}$ from a braid word $g$ to a braid word $h$ and we need to prove the identity $R\left(S_{2}\right)= \pm R\left(S_{1}\right)$, i.e. that $R\left(S_{2}\right)$ is homotopic to either $R\left(S_{1}\right)$ or $-R\left(S_{1}\right)$.

Movie moves 1-10 are easy to take care of. In each of these moves the movies $S_{1}$ and $S_{2}$ are invertible (have no branch points), being a sequence of braid Reidemeister moves, and the corresponding bimodule homomorphisms $R\left(S_{1}\right), R\left(S_{2}\right)$ are invertible. Two isomorphisms $R\left(S_{1}\right), R\left(S_{2}\right)$ between invertible graded $A_{n^{-}}$ bimodules $R(g), R(h)$ differ by multiplication by an invertible degree 0 central element of $A_{n}$. Hence $R\left(S_{2}\right)= \pm R\left(S_{1}\right)$. (This argument was used in [Kh2, BN] and $[\mathbf{S t 1}]$ when proving that certain invariants of tangle cobordisms are well-defined up to rescaling.)

Movie move 15 (locality moves) follow at once from the following observation. Given four bimodules over a ring $A$ and bimodule homomorphisms $u: N_{1} \longrightarrow N_{2}$ and $v: N_{3} \longrightarrow N_{4}$, the bimodule homomorphism $u \otimes v: N_{1} \otimes N_{3} \longrightarrow N_{2} \otimes N_{4}$ can be written as $(u \otimes \mathrm{Id}) \circ(\mathrm{Id} \otimes v)$, as well as (Id $\otimes v) \circ(u \otimes \mathrm{Id})$.

Movie move 11 follows easily, since the canonical isomorphisms of bimodule complexes $R_{i} \otimes R_{j} \cong R_{j} \otimes R_{i}, R_{i} \otimes R_{j}^{\prime} \cong R_{j}^{\prime} \otimes R_{i}$, etc. are compatible with the homomorphisms $w_{1}, \ldots, w_{4}$.

This leaves us with braid movie moves 12-14. Each of the two movies in each of these three moves contains a unique type II transformation, which could be either positive or negative.

We first deal with the simpler case of a positive type II transformation. Then the two movies $S_{1}, S_{2}$ in any of the movie moves $12-14$ go from a braid word $g$ to a braid word $h$ obtained from $g$ by either inserting a positive braid $\sigma_{i}$ or deleting a negative braid $\sigma_{i}^{-1}$. Each movie $S_{1}, S_{2}$ is a composition of Reidemeister braid moves and a single homomorphism (either $w_{1}$ or $w_{2}$, see above) of bimodule complexes. It is easy to check that the space of grading-preserving homomorphisms from $R(\emptyset)$ to $R\left(\sigma_{i}\right)$ 
is $\mathbb{Z}$, with generator $w_{1}$. Likewise, the space of grading-preserving homomorphisms from $R\left(\sigma_{i}^{-1}\right)$ to $R(\emptyset)$ is $\mathbb{Z}$, with generator $w_{2}$. This implies that the space of gradingpreserving homomorphisms from $R(g)$ to $R(h)$ is $\mathbb{Z}$, with $R\left(S_{1}\right), R\left(S_{2}\right)$ both being generators. Hence $R\left(S_{2}\right)= \pm R\left(S_{1}\right)$, which takes care of movie moves 12-14 in the positive case.

All possible versions of the movie move 12 with the negative type II transformation follow by direct computation. A sample computation for the version in Figure 6 is included below.

The maps $F\left(S_{1}\right), F\left(S_{2}\right)$ go from the bimodule $A_{n}$ to $R_{i}^{\prime}$. The first map is given by the composition written below

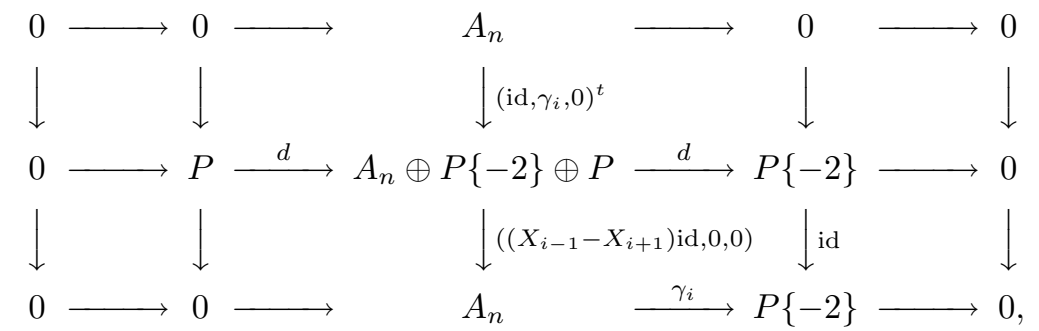

where $P$ is a shorthand for $P_{i} \otimes{ }_{i} P$, while the map $F\left(S_{2}\right)$ is just $w_{3}$. Clearly, $F\left(S_{1}\right)$ $=F\left(S_{2}\right)$.

To treat movie moves $13-14$ with a negative type II transformation we need to do some preliminary work.

Given an endofunctor $F$ on $\mathcal{C}\left(A_{n}\right)$ and a central element $a \in A_{n}$, we denote by $l_{a}$ the natural transformation $F \longrightarrow F$ given by left multiplication by $a$ :

$$
F \cong \operatorname{Id} \circ F \stackrel{a \circ \operatorname{id}_{F}}{\longrightarrow} \operatorname{Id} \circ F \cong F .
$$

Likewise, denote by $r_{a}$ the natural transformation $F \longrightarrow F$ given by right multiplication by $a$ :

$$
F \cong F \circ \operatorname{Id~} \stackrel{\operatorname{id}_{F} \circ a}{\longrightarrow} F \circ \operatorname{Id} \cong F .
$$

Suppose now that $a$ is a linear combination of $X_{j} \mathrm{~s}$,

$$
a=\sum_{j=1}^{n} a_{j} X_{j}
$$

and take $F=R_{i}$ or $R_{i}^{\prime}$.

Proposition 3.4. For any $a$ as above and $1 \leqslant i \leqslant n$,

$$
\text { and } \begin{aligned}
\left(l_{a}-r_{a-a_{i}\left(X_{i-1}+2 X_{i}+X_{i+1}\right)}\right) R_{i} & =0 \\
\left(l_{a}-r_{a-a_{i}\left(X_{i-1}+2 X_{i}+X_{i+1}\right)}\right) R_{i}^{\prime} & =0
\end{aligned}
$$

in $\operatorname{Fun}\left(A_{n}\right)$.

In other words, the endomorphism $l_{a}-r_{a-a_{i}\left(X_{i-1}+2 X_{i}+X_{i+1}\right)}$ of the complexes $R_{i}$ and $R_{i}^{\prime}$ of bimodules is zero homotopic. 

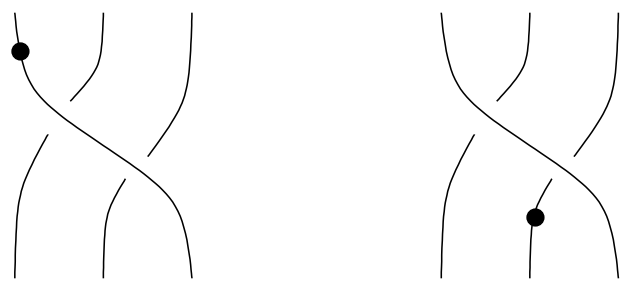

Figure 11: The endomorphisms $l_{X_{1}}$ and $r_{X_{1}+X_{2}}$ of $R_{1}^{\prime} \otimes R_{2}^{\prime}$

Proof. Consider the homotopy $h$ from $R_{i}$ to itself which takes $A_{i}$ to $P_{i} \otimes{ }_{i} P$ via $\gamma_{i}$ and $P_{i} \otimes{ }_{i} P$ to 0 . Then the zero homotopic endomorphism $h d+d h$ of $R_{i}$ is given on generators $(i) \otimes(i) \in P_{i} \otimes{ }_{i} P$ and $1 \in A_{n}$ by

$$
\begin{aligned}
(h d+d h)((i) \otimes(i)) & =X_{i} \otimes(i)+(i) \otimes X_{i}, \\
(h d+d h)(1) & =X_{i-1}+2 X_{i}+X_{i+1} .
\end{aligned}
$$

Next, we compute

$$
\begin{aligned}
\left(l_{a}-r_{a-a_{i}\left(X_{i-1}+2 X_{i}+X_{i+1}\right)}\right)((i) \otimes(i)) & =a_{i} X_{i} \otimes(i)+a_{i}(i) \otimes X_{i}, \\
\left(l_{a}-r_{a-a_{i}\left(X_{i-1}+2 X_{i}+X_{i+1}\right)}\right)(1) & =a_{i}\left(X_{i-1}+2 X_{i}+X_{i+1}\right) .
\end{aligned}
$$

Therefore,

$$
\left(l_{a}-r_{a-a_{i}\left(X_{i-1}+2 X_{i}+X_{i+1}\right)}\right) R_{i}=a_{i}(h d+d h) R_{i},
$$

and the left-hand side is homotopic to zero. A similar argument works for $R_{i}^{\prime}$, using $\beta_{i}$ in the homotopy.

Remark. When $i=1$ or $i=n$ we should omit $X_{i-1}$, respectively $X_{i+1}$, from the above formulas and computations.

Remark. The braid group action on $\mathcal{C}\left(A_{n}\right)$ descends to an action on the center of $A_{n}$ which happens to factor through the action of the symmetric group and is given by the above formulas. The generator $s_{i}$ of the symmetric group takes $a$ to $a-a_{i}\left(X_{i-1}+2 X_{i}+X_{i+1}\right)$. This action also appears, in a similar context, in [FKS, Section 6].

Corollary 3.5. The following endomorphisms of the bimodule complexes $R_{i}$ and $R_{i}^{\prime}$ are null-homotopic:

$$
l_{X_{i-1}+X_{i}}+r_{X_{i}+X_{i+1}}, \quad l_{X_{i}+X_{i+1}}+r_{X_{i-1}+X_{i}}, \quad l_{X_{i-1}-X_{i+1}}+r_{X_{i+1}-X_{i-1}} .
$$

We depict the endomorphisms $l_{X_{i-1}+X_{i}}$ and $r_{X_{i-1}+X_{i}}$ of a bimodule complex associated with a braid word by a dot placed near the end of the $i$-th top, respectively bottom, strand; see an example in Figure 11. One of the cases of the corollary is written in Figure 12. 


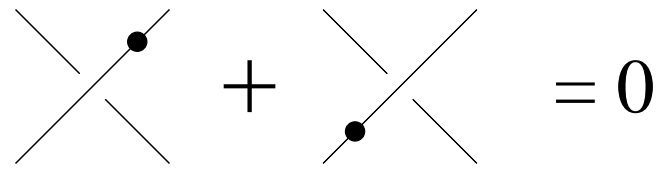

Figure 12: The endomorphisms $l_{X_{i}+X_{i+1}}$ and $-r_{X_{i-1}+X_{i}}$ of $R_{i}$ are chain homotopic.
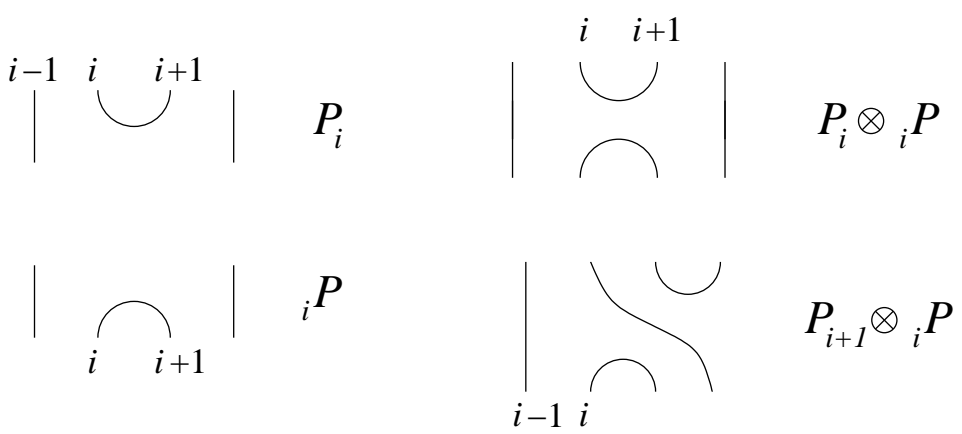

Figure 13: Graphical notation for projective modules $P_{i},{ }_{i} P$ and their tensor products.

We now consider the instance of braid movie move 13 depicted in Figure 6 and, for convenience, introduce additional graphical notation in Figure 13.

Next, we rewrite $F\left(S_{1}\right)$ in graphical form, as in Figure 14. The map $\nu_{0}$ is right multiplication by $X_{i-1}-X_{i+1}$. The second row in the figure presents the complex $R\left(\sigma_{i}^{-1} \sigma_{i+1}^{-1} \sigma_{i}^{-1}\right)$ as the cone of the map of complexes

$$
0 \longrightarrow R\left(\sigma_{i}^{-1} \sigma_{i+1}^{-1}\right) \stackrel{\rho_{1}}{\longrightarrow} R\left(\sigma_{i}^{-1} \sigma_{i+1}^{-1}\right) \otimes_{A_{n}} P_{i} \otimes{ }_{i} P \longrightarrow 0,
$$

where $\rho_{1}=\operatorname{Id} \otimes \gamma_{i}$. The map $\pi_{1}$ is a homotopy equivalence of complexes, given by taking the quotient of $R\left(\sigma_{i}^{-1} \sigma_{i+1}^{-1}\right) \otimes_{A_{n}} P_{i} \otimes{ }_{i} P$ by a contractible direct summand. The map $\nu_{1}$ is a homotopy equivalence. The map $\nu_{2}^{-1}$ is the inverse of the quotient map $\nu_{2}$, depicted in the lower half of Figure 15. The map $\nu_{2}$ is the identity on $R\left(\sigma_{i}^{-1} \sigma_{i+1}^{-1}\right)=R_{i}^{\prime} \otimes R_{i+1}^{\prime}$ and the quotient map $\pi_{2}$ (analogous to $\pi_{1}$ ) on the second term of the cone. Since $\nu_{2}$ is a homotopy equivalence, its inverse is well-defined.

In Figure 15 we depict the composition of $F\left(S_{2}\right)$ with $\nu_{2}$. The map $\nu_{3}$ is left multiplication by $X_{i}-X_{i+2}$. The equation $F\left(S_{1}\right)= \pm F\left(S_{2}\right)$ is equivalent to $\nu_{2} F\left(S_{1}\right)$ $= \pm \nu_{2} F\left(S_{2}\right)$. The map $\nu_{2} F\left(S_{1}\right)$ is right multiplication by $X_{i-1}-X_{i+1}$ and $\nu_{2} F\left(S_{2}\right)$ is left multiplication by $X_{i}-X_{i+2}$; see Figure 16 . The endomorphisms $r_{X_{i-1}-X_{i+1}}$ and $-l_{X_{i}-X_{i+2}}$ of $R_{i}^{\prime} \otimes R_{i+1}^{\prime}$ are chain homotopic (apply Corollary 3.5 several times), via a degree 2 homotopy $h$. We need to show that $r_{X_{i-1}-X_{i+1}}+l_{X_{i}-X_{i+2}}$ is null-homotopic when viewed as a homomorphism from $R_{i}^{\prime} \otimes R_{i+1}^{\prime}$ to the complex depicted at the bottom of Figure 16. 
Homology, Homotopy and Applications, vol. 9(2), 2007

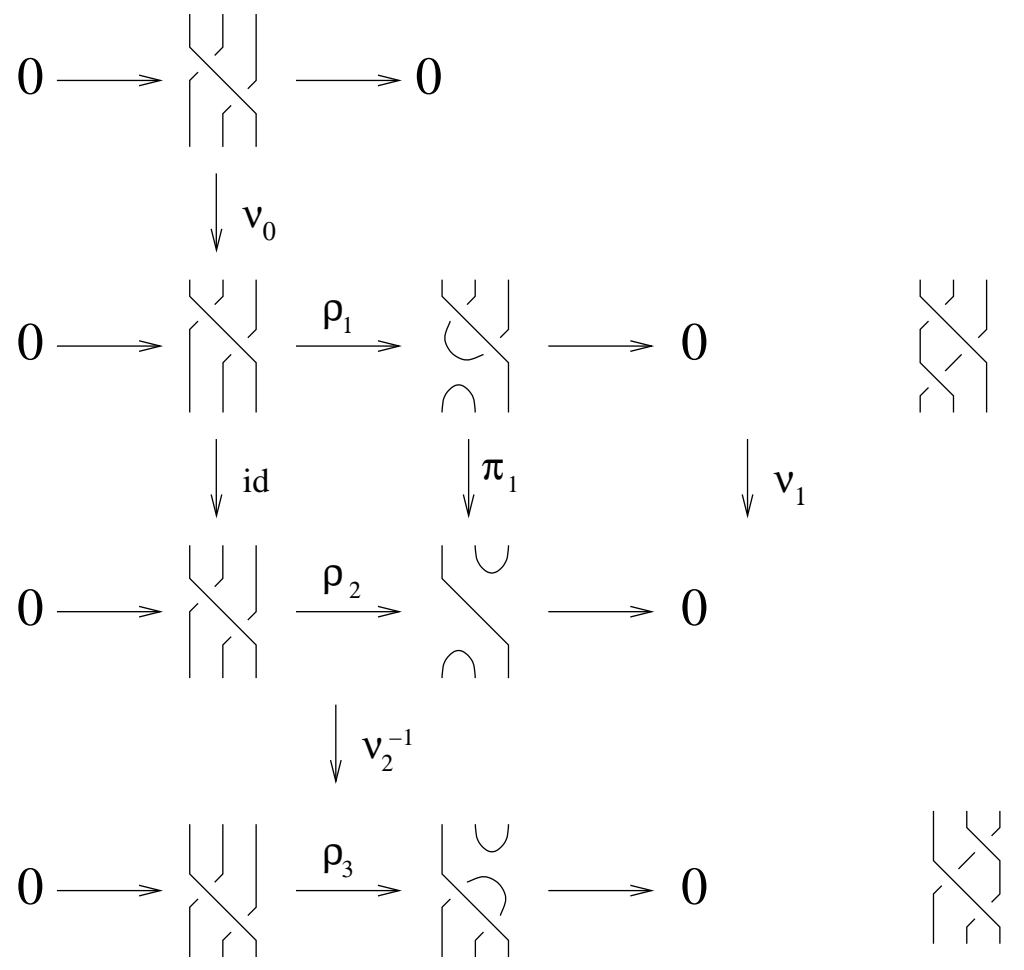

Figure 14: The map $F\left(S_{1}\right)$ in move 13 presented in graphical form.
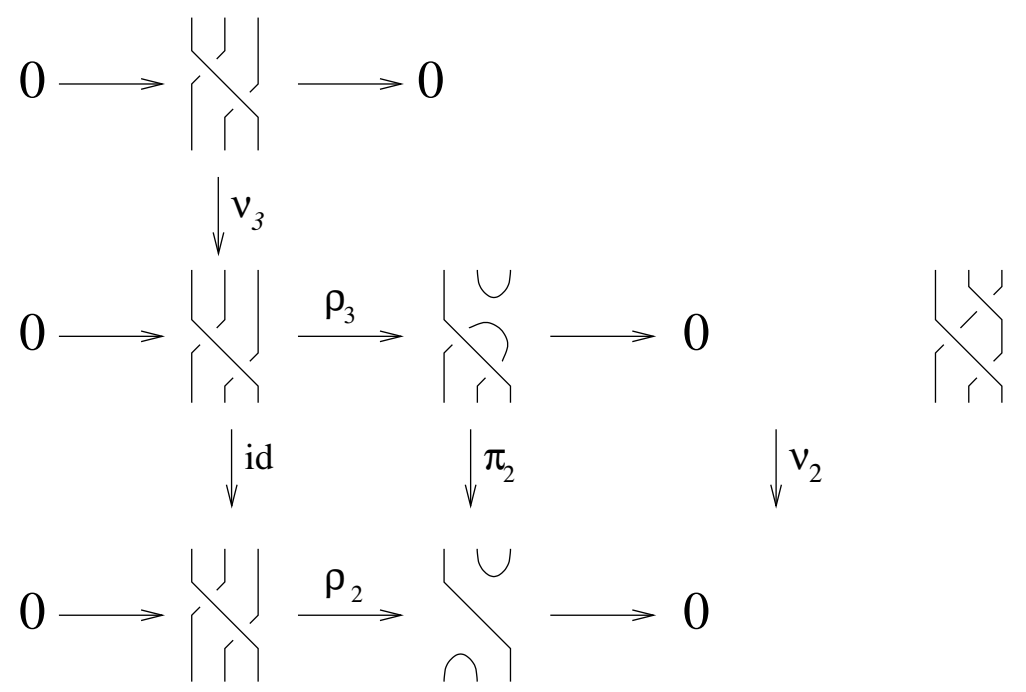

Figure 15: The map $\nu_{2} F\left(S_{2}\right)$ in move 13 written in graphical form. 
Homology, Homotopy and Applications, vol. 9(2), 2007

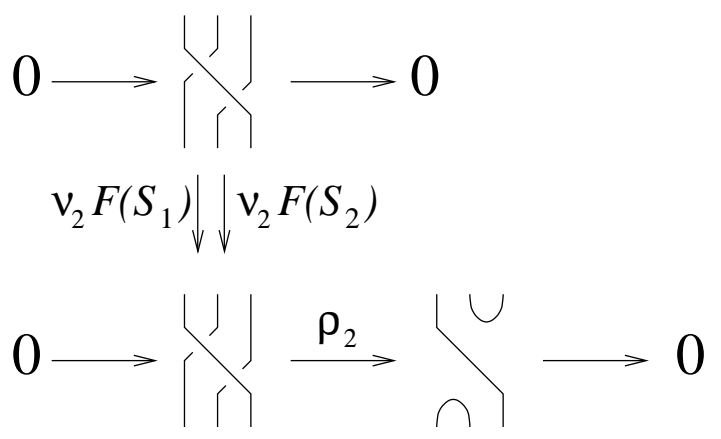

Figure 16: The maps $\nu_{2} F\left(S_{1}\right)$ and $\nu_{2} F\left(S_{2}\right)$.

A direct but nontrivial computation, which requires explicitly writing down the complex $R_{i}^{\prime} \otimes R_{i+1}^{\prime}$ and which we leave to the reader, shows that the relation

$$
d h+h d=r_{X_{i-1}-X_{i+1}}+l_{X_{i}-X_{i+2}}
$$

implies $\rho_{2} h=0$ as a map of abelian groups. Consequently,

$$
\nu_{2}\left(F\left(S_{1}\right)+F\left(S_{2}\right)\right)=r_{X_{i-1}-X_{i+1}}+l_{X_{i}-X_{i+2}}
$$

is null-homotopic as a map between the complexes in Figure 16. Thus, $F\left(S_{1}\right)$ $+F\left(S_{2}\right)=0$ and this instance of braid movie move 13 follows.

Three other cases of movie move 13 with a negative branch point and with the top frame being one of the braids $\sigma_{i} \sigma_{i+1}, \sigma_{i+1} \sigma_{i}, \sigma_{i+1}^{-1} \sigma_{i}^{-1}$ (instead of $\sigma_{i}^{-1} \sigma_{i+1}^{-1}$ ) follow via nearly identical arguments. The remaining instances of movie move 13 with a negative branch point are obtained by reading the frames in the above four cases from bottom to top. These instances follow (on the topological level) from the first four cases and from some of the moves 1-12.

Finally, we deal with braid movie move 14. The instance of this move shown in Figure 6 is equivalent to the move depicted in Figure 17 (we formed the product of this move with the identity cobordism on $\sigma_{i}^{-1}$ ).

Look at the branch point indicated by the two triangles on the top strand of the left-hand movie of Figure 17. Move 13 allows us to drag this branch point up and across the third strand of the braid. Thus, the left-hand movie is equivalent to the movie where the branch point is created to the right of this strand, just like in the other movie in Figure 17. These two movies are equivalent via a composition of several earlier moves. Other cases of move 14 follow in the same fashion, implying that move 14 is redundant. A complete derivation of move 14 from move 13 and other moves can be found in Figure 18, kindly provided to us by Scott Carter.

Our proof of Theorem 1.1 ends here.

\subsection{Categorification via Rouquier complexes}

Consider the polynomial algebra $k\left[x_{1}, \ldots, x_{n}\right]$ over a field $k$ and its subalgebra $A=k\left[x_{1}-x_{2}, x_{2}-x_{3}, \ldots, x_{n-1}-x_{n}\right]$ of polynomials in $x_{i}-x_{i+1}, 1 \leqslant i \leqslant n-1$. 

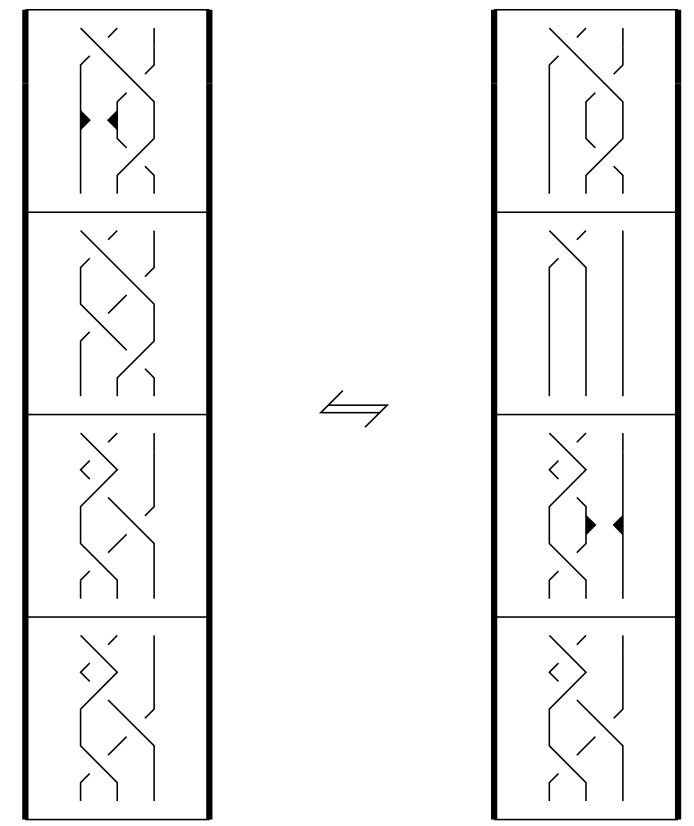

Figure 17: A modified braid movie move 14.

The symmetric group $S_{n}$ acts on the set $\left\{x_{1}, \ldots, x_{n}\right\}$ by permuting the indices, and we consider the induced action on $A$. Let $A^{i}$ be the subalgebra of polynomials invariant under the action of the permutation $s_{i}=(i, i+1)$. We make $A$ and $A^{i}$ graded by giving each $x_{j}$ degree 1 , and consider the following complexes $R_{i}$ and $R_{i}^{\prime}$ of graded $A$-bimodules:

$$
\begin{aligned}
& R_{i}: \quad 0 \longrightarrow A \otimes_{A^{i}} A \stackrel{m}{\longrightarrow} A \longrightarrow 0 \longrightarrow 0, \\
& R_{i}^{\prime}: \quad 0 \longrightarrow 0 \longrightarrow A \stackrel{\eta_{i}}{\longrightarrow} A \otimes_{A^{i}} A\{-1\} \longrightarrow 0,
\end{aligned}
$$

with $A$ in cohomological degree 0 in both complexes, $m$ the multiplication map, and

$$
\eta_{i}(1)=\left(x_{i}-x_{i+1}\right) \otimes 1+1 \otimes\left(x_{i}-x_{i+1}\right) .
$$

Rouquier [Ro1] introduced these complexes (for an arbitrary Coxeter group) and showed that they induce a genuine braid group action on the homotopy category of complexes of graded $A$-modules.

We now extend Rouquier's construction to an invariant of braid cobordisms. To the two movies of a positive double point (see Figure 9) we assign the homomorphisms

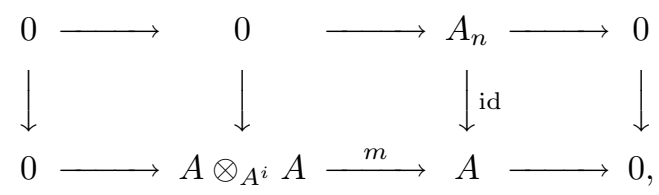



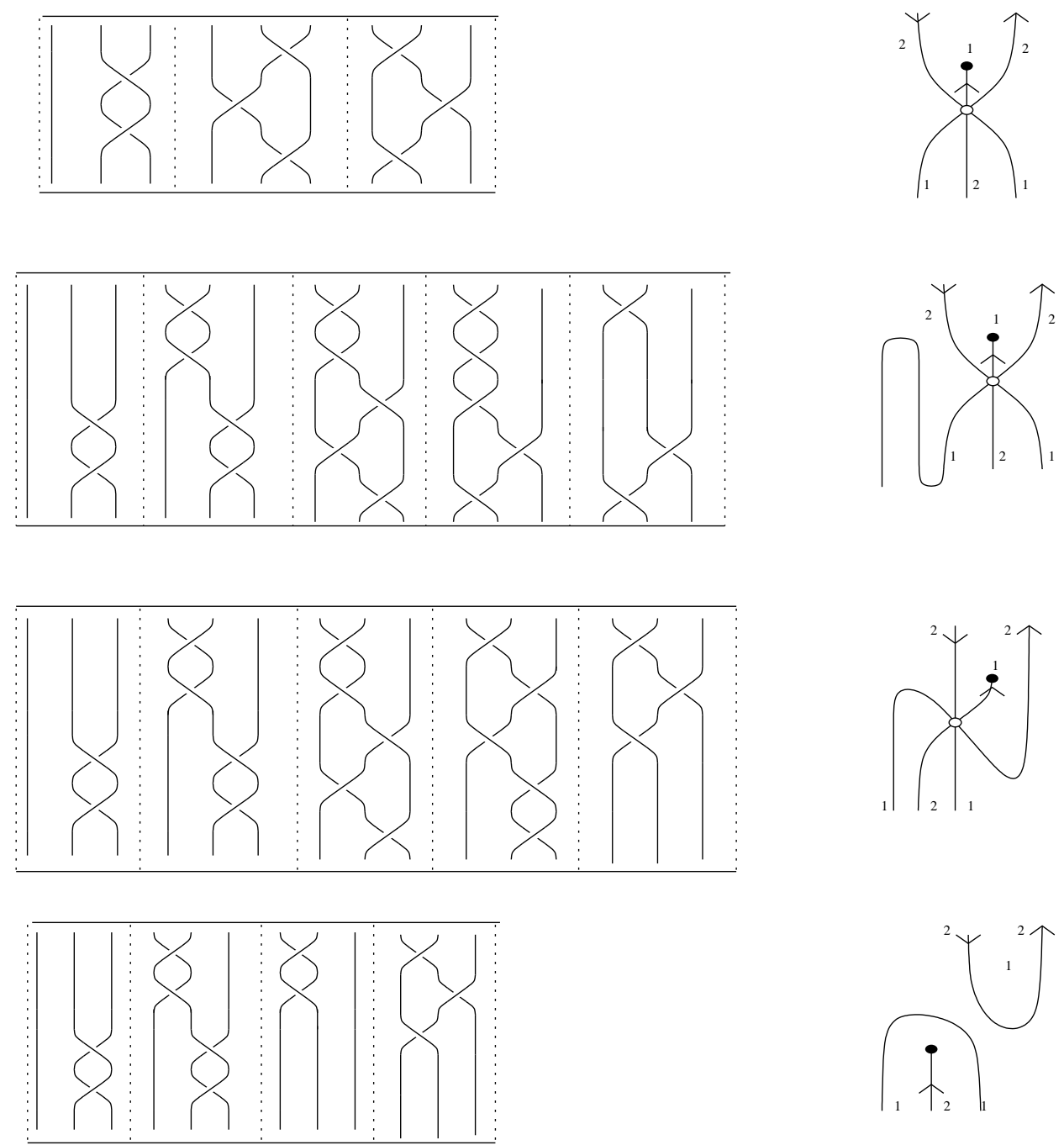

Figure 18: (courtesy of Scott Carter) Move 14 follows from the other moves. Diagrams on the right-hand side are notations in [CS2] for braid cobordisms. 
and

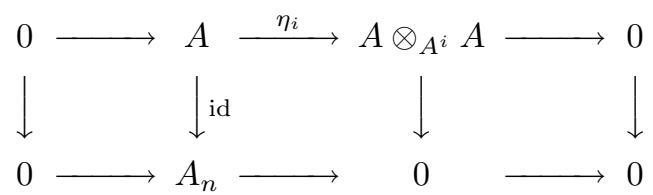

of the corresponding bimodule complexes. To a negative double point we should assign a homomorphism from $A$ to (possibly shifted) $R_{i}^{\prime}$. Since $\eta_{i}$ is injective, the only bimodule homomorphism from $A$ to $R_{i}^{\prime}$ is zero, while any homomorphism from $A$ to $R_{i}^{\prime}[-1]$ is null-homotopic. Thus, we are forced to assign the zero homomorphism of complexes to any braid cobordism which contains a negative branch point.

Proposition 3.6. The above assignment is a semi-trivial categorification of a braid group representation.

Proof. Indeed, we know from [Ro1] that the complexes $R_{i}, R_{i}^{\prime}$ produce a braid group action. Arguments analogous to the ones in the first half of the proof of Theorem 1.1 imply that the braid movie move relations hold and our data extends to a projective monoidal functor from $\mathcal{B C}_{n}^{c}$ to $\operatorname{Fun}(\mathcal{C}(A)$ ), where $\mathcal{C}(A)$ is the homotopy category of complexes of graded $A$-modules. If we restrict to bounded complexes of finitely-generated graded $A$-modules, then the Grothendieck group will be a rank one free $\mathbb{Z}\left[q, q^{-1}\right]$-module with generator $[A]$, and $\sigma_{i}$ will act by $\sigma_{i}[A]=-q[A]$.

Remark. The categorification is semi-trivial since any negative braid cobordism has the trivial invariant. Such terminology does not do justice to this categorification, since the bimodules in $R_{i}$ and their tensor products carry an enormous amount of information, can be used to reconstruct a regular block of the category $\mathcal{O}$ for $\mathfrak{s l}_{n}$ (see [So]) and serve as building blocks for a triply-graded link homology theory [Kh5].

Remark. Since we can work over $\mathbb{Z}$ rather than over $k$, the projectivity is at most in the minus sign.

The above example of a braid group action led Rouquier to define the notion of a braid group categorification (working in the context of Coxeter braid groups; we specialize his construction to the case of the usual braid group $\mathrm{Br}_{n}$ ). For each braid $\sigma$ choose a representative $R(\sigma)$ in the isomorphism class of complexes of graded $A$ bimodules assigned to $\sigma$. Rouquier considers a monoidal category $\mathcal{B}_{S_{n}}$ with objects $\sigma$ and graded bimodule homomorphisms $\operatorname{Hom}(R(\sigma), R(\tau))$ as morphisms from $\sigma$ to $\tau$. He then defines a braid group categorification as a monoidal functor from $\mathcal{B}_{S_{n}}$ to $\operatorname{Fun}(\mathcal{C})$, for a triangulated category $\mathcal{C}$.

Recall that our definition of a categorification of a braid group representation requires a (projective) monoidal functor from $\mathcal{B C}_{n}$ to $\operatorname{Fun}(\mathcal{C})$. In both categories, $\mathcal{B C}_{n}$ and $\mathcal{B}_{S_{n}}$, isomorphism classes of objects are in bijection with braids. For $\mathcal{B}_{S_{n}}$ this is a nontrivial result, which says that $R(\sigma) \cong R(\tau)$ implies $\sigma=\tau$; see [Ro1, Remark 3.9]. The morphisms in the two categories are quite different, though. In $\mathcal{B C}_{n}$ they are braid cobordisms, a purely topological construct, while in $\mathcal{B}_{S_{n}}$ they are homomorphisms of certain bimodules. 
A few paragraphs above we defined a (projective) monoidal functor from $\mathcal{B C}_{n}$ to $\mathcal{B}_{S_{n}}$, trivial on any cobordism which contains a negative branch point. Using this functor we can convert any braid group categorification in the sense of Rouquier into a semi-trivial categorification of a braid group representation in our sense. This conversion is the closest relation that we were able to find between Rouquier's notion of categorification of braid group representations and ours.

\subsection{Categorification in Fukaya-Floer categories}

A semi-trivial action of the braid cobordism category. Braid group $\mathrm{Br}_{n}$ actions arise naturally in symplectic geometry as groups of symplectomorphisms generated by Dehn twists about $A_{n-1}$-chains of Lagrangian spheres [Se2, Appendix]. Following $[\mathbf{K S}]$ we describe the local model as monodromy on Milnor fibers of $k$-dimensional $A_{n-1}$ singularities (for any $k$ ). Passing to the derived Fukaya-Floer category one gets braid group actions on triangulated categories, categorifying the usual monodromy representations on (co)homology. We then describe an extension to a semi-trivial representation of the braid cobordism category. Throughout we are assuming that all the technicalities involved in the existence of the (derived) Fukaya-Floer category are overcome; since our local examples are exact symplectic manifolds with $c_{1}=0$, Paul Seidel's forthcoming book [Se6] justifies this carefully (and allows us to use Floer cohomology consisting of finite-dimensional graded $\mathbb{C}$ vector spaces, rather than relatively or periodically graded modules over Novikov rings), but there are probably parts of the discussion below that available technical results do not yet quite fully justify.

Thinking of $\mathrm{Br}_{n}$ as the fundamental group of the configuration space $C_{n}$ of $n$ unordered points $\mathbf{p}=\left\{p_{j}\right\}_{j=1}^{n}$ in $\mathbb{C}$, we can associate a $k$-dimensional symplectic manifold to every point $\mathbf{p} \in C_{n}$ :

$$
X_{\mathbf{p}}=\left\{\left(x_{1}, \ldots, x_{k}, t\right) \in \mathbb{C}^{k} \times \mathbb{C}: \sum_{i=1}^{k} x_{i}^{2}=\prod_{j=1}^{n}\left(t-p_{j}\right)\right\} .
$$

We give $X_{\mathbf{p}}$ the symplectic structure $\omega$ that it inherits from $\mathbb{C}^{k+1}$. Via the $t$ variable $X_{\mathbf{p}}$ fibers over $\mathbb{C}$ with fibers affine quadrics $\sum_{i=1}^{k} x_{i}^{2}=a$ (which are symplectically just $T^{*} S^{k-1} \mathrm{~s}$ ) except at the critical points $t=p_{j}$ where the $S^{k-1}$ s collapse to points in the singular fibers $\sum_{i=1}^{k} x_{i}^{2}=0$.

Therefore for every loop in $C_{n}$ we get an $S^{1}$-family of such symplectic manifolds with a fiberwise symplectic closed 2-form on the total space. There is a natural connection on the bundle whose horizontal subspaces are the orthogonals, with respect to the 2-form, to the fibers; see [Se4] or [Se5]. The induced symplectic parallel transport along the fibration gives us monodromy in the symplectomorphism $\operatorname{group} \operatorname{Aut}\left(X_{\mathbf{p}}, \omega\right)$. (There is a technical issue here due to the noncompactness of the fibers; this is resolved in a related example in [SS, Section $4 \mathrm{~A}]$.) Isotopic loops give hamiltonian isotopic symplectomorphisms (since the curvature of the connection is hamiltonian) producing a homomorphism

$$
\operatorname{Br}_{n}=\pi_{1}\left(C_{n}\right) \rightarrow \pi_{0}\left(\operatorname{Aut}\left(X_{\mathbf{p}}, \omega\right)\right) .
$$

In particular the positive generators of $\mathrm{Br}_{n}$ can be described as follows. Order the 
points $p_{j} \in \mathbb{C}$ and pick paths in $\mathbb{C}$ between consecutive points, which do not intersect in their interiors. Over these paths lie canonical Lagrangian spheres $S^{k} \subset X_{\mathbf{p}}$ (fibered by the $S^{k-1} \mathrm{~s}$ in the $T^{*} S^{k-1}$ fibers of $X_{\mathbf{p}}$ ). Then rotating two adjacent points anticlockwise through 180 degrees about the path between them gives rise to the monodromy transformation that is the Dehn twist about the associated Lagrangian sphere. This can be thought of as monodromy around the singular variety one gets by collapsing this Lagrangian sphere by moving the two points together. (In other words we can fill in the $S^{1}$-family to a family over the disc $D^{2}$ whose central fiber has an ordinary double point given by setting two $p_{j}$ s to be equal in (17).)

Roughly speaking, symplectomorphisms of $X_{\mathbf{p}}$ act on the Fukaya-Floer category of $X_{\mathrm{p}}$ since the latter is a symplectic invariant constructed out of the Lagrangian submanifolds of $X_{\mathbf{p}}$ and their Floer cohomologies. More precisely, since $c_{1}\left(X_{\mathbf{p}}\right)$ $=0$, there is an $A^{\infty}$-category whose objects are those graded Lagrangian submanifolds [Se3] (carrying flat unitary bundles) whose Floer cohomology is well-defined [FO3]. Seidel also shows that the above Dehn twists furnish graded symplectomorphisms [Se3], and so act (as equivalences) on the derived Fukaya-Floer category $D^{b}\left(F u k\left(X_{\mathbf{p}}\right)\right)$ of $X_{\mathbf{p}}$. Hamiltonian isotopies between symplectomorphisms induce natural transformations between their corresponding functors on $D^{b}\left(F u k\left(X_{\mathbf{p}}\right)\right)$ (see below), so we end up with an induced representation

$$
\operatorname{Br}_{n}=\pi_{1}\left(C_{n}\right) \rightarrow \pi_{0}\left(\operatorname{Aut}\left(X_{\mathbf{p}}, \omega\right)\right) \rightarrow \operatorname{Auteq}\left(D^{b}\left(F u k\left(X_{\mathbf{p}}\right)\right)\right) .
$$

In particular, the action of the Dehn twist $T_{L}$ about the Lagrangian sphere $L$ (the twist being independent of the grading on $L)$ on the object $\left[L^{\prime}\right] \in D^{b}\left(F u k\left(X_{\mathbf{p}}\right)\right)$ can be described as follows [Se4]. Think of the identity in $H F^{*}\left(L, L^{\prime}\right)^{*} \otimes H F^{*}\left(L, L^{\prime}\right)$ as a morphism from $H F^{*}\left(L, L^{\prime}\right) \otimes[L]$ to $\left[L^{\prime}\right]$ in $D^{b}\left(F u k\left(X_{\mathbf{p}}\right)\right)$, and take its cone:

$$
T_{L}\left[L^{\prime}\right]=\operatorname{Cone}\left(H F^{*}\left(L, L^{\prime}\right) \otimes L \rightarrow L^{\prime}\right) .
$$

(See (77), which is what this should correspond to under mirror symmetry.)

We explain how this can be extended to a semi-trivial representation of the braid cobordism category. For any braid cobordism with a negative braid move we set to zero the natural transformation between the two corresponding functors on $D^{b}\left(F u k\left(X_{\mathbf{p}}\right)\right)$. Braid cobordisms with no positive or negative moves (i.e. isotopies between two braids) induce natural transformations between the two functors associated to those braids (as claimed above) as follows. The isotopy gives a family of symplectic manifolds $X_{\mathbf{p}}$ over the annulus, with the two boundary $S^{1}$-families being those associated to the two braids. Call their monodromies $\psi$ and $\phi$. The family carries a closed 2-form which is fiberwise symplectic; we add on a large multiple of the pullback of a symplectic form on the annulus to produce a symplectic total space. Then Seidel [Se5] defines what he calls the relative Gromov invariant of this family, counting pseudoholomorphic sections (in an appropriate compatible almost complex structure) interpolating between horizontal sections over each boundary (i.e. generators of the Floer cochain complexes of the boundary symplectomorphisms $\psi$ and $\phi)$ to produce a canonical element of the Floer cohomology $H F^{*}\left(\psi^{-1} \circ \phi\right)$ of the symplectomorphism $\psi^{-1} \circ \phi$. This element provides the required natural transformation by pairing it with $1 \in H F^{0}(\psi(L), \psi(L))$ using the cup product in Floer 
cohomology:

$$
H F^{*}(\psi(L), \psi(L)) \otimes H F^{*}\left(\phi \circ \psi^{-1}\right) \rightarrow H F^{*}(\psi(L), \phi(L)) .
$$

(This is best described, as Ivan Smith pointed out to us, on the product $\left(X_{\mathbf{p}}, \omega\right)$ $\times\left(X_{\mathbf{p}},-\omega\right)$, where the above groups become $H F^{*}\left(\psi\left(L_{1}\right) \times \psi\left(L_{2}\right), \Delta_{X_{\mathbf{p}}}\right)$, $H F^{*}\left(\Delta_{X_{\mathrm{p}}}, \Gamma_{\phi \circ \psi^{-1}}\right)$, and $H F^{*}\left(\psi\left(L_{1}\right) \times \psi\left(L_{2}\right), \Gamma_{\phi \circ \psi^{-1}}\right)$ respectively, and the cup product becomes the ordinary cup product on Lagrangian Floer cohomology defined by holomorphic triangles. Here $\Delta_{X_{\mathbf{p}}} \subset X_{\mathbf{p}} \times X_{\mathbf{p}}$ is the diagonal Lagrangian, and $\Gamma_{\phi \circ \psi^{-1}}$ is the graph of $\phi \circ \psi^{-1}$.)

Given a braid cobordism which is a composition of braid isotopies and positive braid moves, there is a corresponding fibration over the annulus, the fibers of which are either manifolds or have complex ordinary double points. This is because the family (17) over $C_{n}$ extends by the same formulae over the partial compactification $\bar{C}_{n}$ where pairs of points are allowed to collide, over which $X_{p}$ acquires an ordinary double point. The local model (9) for positive braid moves corresponds [Ru] locally to a holomorphic map from the base (the $z_{1}$ axis in (9)) to $\bar{C}_{n}$ that intersects the double point locus transversally and positively. So for an appropriate choice of oriented complex structure on our annulus the pullback family (17), with the closed 2 -form pulled back from $\mathbb{C}^{k+1}$, is a Lefschetz fibration over the annulus in the sense of [Se5, Definition 1.5].

Adding a large oriented symplectic form pulled back from the annulus, we get a Lefschetz fibered symplectic manifold. Using pseudoholomorphic sections of this Lefschetz fibration with an appropriate almost complex structure, Seidel extends the Gromov invariant to such fibrations [Se5, Section 3] to give a chain map between the Floer cochain complexes of the two boundary symplectomorphisms. This is our natural transformation.

For instance, in the case of the braid cobordism with one positive braid move from the identity to the Dehn twist about a Lagrangian sphere $L$, we get the natural transformation from the identity to $T_{L}$ that comes from mapping $\left[L^{\prime}\right]$ into the second term of (18) (inducing, for instance, the horizontal map of [Se5, Theorem 1]). This is the analogue for Fukaya categories of the positive natural transformations (105).

Since all of the positive braid movie moves of Section 3.1 arise from isotopies of maps of the annulus into $\bar{C}_{n}$ through maps which are orientedly transverse to the double point locus, they give rise to isomorphic natural transformations. This is because in Seidel's theory, two homotopies between symplectomorphisms that are themselves homotopic induce homotopic maps on Floer cochain complexes.

We do not currently know a natural way to extend this nontrivially to negative branch points, as the geometry involved would be locally anti-holomorphic. Seidel and Smith have some ideas about how one might proceed, however.

The Fukaya-Floer category of $T^{*} F l$. In discussions with Paul Seidel in 1998 about the braid group action on the derived category of coherent sheaves on the cotangent bundle of the flag variety of Section 4 , he explained what should be the translation of the action under mirror symmetry. The $N_{i}$ of Section 4 are coisotropic submanifolds (in the standard symplectic structure on $T^{*} \mathrm{Fl}$ ) whose characteristic foliation is a fibration by isotropic $S^{2}$ s; therefore one can do generalised Dehn twists 
about them ([Se1, Section 1.4], [Pe, Section 2.3]), yielding symplectomorphisms $T_{i}$. Seidel conjectures that these satisfy the braid relations, and so give a representation of the braid group on the Fukaya category of $K$.

One should be able to extend this to a semi-trivial action of the braid cobordism category in the same way as described above for the manifolds $X_{\mathbf{p}}$. In place of the ordinary double point degenerations of the $X_{\mathbf{p}}$ one would use normal crossings degenerations of $T^{*} F l$ in which the $N_{i}$ collapse to the bases $K_{i}$ of their characteristic $S^{2}$ foliations. This would require a family version of Seidel's theory [Se5].

The examples of Seidel-Smith and Manolescu. There are other naturally occurring families of symplectic manifolds fibering over $C_{n}$ whose Fukaya-Floer categories are related to knot theory and link homology [Kh1, Kh3]. Seidel and Smith [SS] consider a certain nilpotent slice of the set of $\mathfrak{s l}_{2 n}$ matrices, with the fibration to $C_{2 n}^{0}$ (configurations with center of mass zero) given by the set of eigenvalues of the matrix. The symplectic monodromy of the fibers gives a representation of the braid group in the group of symplectomorphisms up to isotopy, and the Seidel-Smith braid invariant is the Floer cohomology of a certain Lagrangian with its image under this representation; they then show that this is in fact a link invariant.

This example is closely related to the example of the Fukaya-Floer category of $T^{*} \mathrm{Fl}$ above, by the deformation of adjoint orbits into the cotangent bundle of the flag variety (by taking the usual simultaneous resolution of the degeneration to the nilpotent cone). Manolescu [Ma1] shows that it is also closely related to the example (17), as it sits inside the Hilbert scheme of points on the two dimensional version of (17). (In this picture, Seidel-Smith's Lagrangian is the product of $n$ of the Lagrangian $S^{2}$ s described above, fibered over $n$ nonintersecting arcs joining disjoint pairs of points in $C_{2 n}$.) In [Ma2] he also produces other more complicated manifolds fibering over configuration space giving rise to more complicated link invariants by the same procedure.

In all of these examples the fibrations extend to fibrations with singular fibers over the partial compactification $\bar{C}_{n}$ in which pairs of points are allowed to coincide. Instead of a Lefschetz fibration with vanishing cycles of Lagrangian spheres collapsing over the discriminant locus to ordinary double points, the example of [SS] exhibits a family version of this behaviour. That is, the singular fibers contain a locus $S$ of singularities, and are locally holomorphically modelled on $S$ times by a surface ordinary double point. The smooth fibers contain coisotropic families of isotropic vanishing $S^{2}$ s fibering over the copy of $S$ to which they collapse under parallel transport to the singular fiber. Something similar happens in [Ma1] (with some extra singularities thrown in for good measure) and in [Ma2], with $S^{2}$ s replaced by $\mathbb{P}^{k}$ and the surface ordinary double points (locally the contraction of $\mathbb{P}^{1} \subset T^{*} \mathbb{P}^{1}$ ) replaced by singularities modelled on the contraction of $\mathbb{P}^{k} \subset T^{*} \mathbb{P}^{k}$.

Thus an extension of Seidel's theory [Se5] from Lefschetz fibrations to fibrations with these fibered singularities would give a family Gromov invariant defining a natural transformation between the symplectic monodromy of a braid (and its associated functor on the Fukaya-Floer category) and that of the braid composed with a simple positive braid cobordism. To reiterate: such a positive braid move corresponds to a map of the annulus into $\bar{C}_{n}$ with one transverse positively oriented 
intersection with the double point locus of $\bar{C}_{n}$. Thus the map can be made holomorphic at this point by an isotopy through maps with the same transversality to the double point locus. Pulling back the family over $\bar{C}_{n}$ and pulling back a large positively oriented symplectic form from the base we get a symplectic manifold fibering over the annulus with one singular fiber with singularity of a fixed standard holomorphic fibered model (compatible with the symplectic form, which is Kähler in a neighbourhood of the singular locus). Denote the symplectic monodromies around the two boundary circles of the annulus by $\psi$ and $\phi$. Then an extension of [Se5] to this setting would provide us with an element of $H F^{*}\left(\phi \circ \psi^{-1}\right)$ and a natural transformation between the actions of $\phi$ and $\psi$ on the derived Fukaya-Floer categories of the fiber.

\subsection{More on polarization}

In our examples of categorifications there tend to exist big discrepancies between the invariants $F(S)$ of positive and negative braid cobordisms $S$. Often, the invariant is nontrivial on any positive braid cobordism but trivial on a negative braid cobordism with just a few branch points. The invariant described in Theorem 1.1 serves as a model example. Recall that to any braid cobordism $S$ between braid words $g$ and $h$ we assigned a homomorphism $\pm F(S)$ of $A_{n}$-bimodule complexes $R(g)$ and $R(h)$.

Proposition 3.7. $F(S)$ is nonzero in the homotopy category for any positive braid cobordism $S$.

Proof. Composing $S$ with the identity cobordism from $g^{-1}$ to itself, we can assume that $g=1$ and $F(S)$ is a homomorphism from $A_{n}$ to the complex $R(h)$. The latter complex consists of a single bimodule $A_{n}$ and the sum of terms isomorphic to $P_{i} \otimes{ }_{j} P$, for various $i$ and $j$. Thus, $R(h)$ decomposes, $R(h) \cong A_{n} \oplus Y(h)$, as a bimodule, not as a complex, where $Y$ is a direct sum of terms $P_{i} \otimes{ }_{j} P$.

Any composition of bimodule maps

$$
A_{n} \longrightarrow P_{i} \otimes{ }_{j} P \longrightarrow A_{n}
$$

takes $1 \in A_{n}$ to the ideal $I$ of $A_{n}$ generated by $X_{1}, \ldots, X_{n}$. Hence, given any chain homotopy $\alpha$ from $A_{n}$ to $R(h), d \alpha+\alpha d$ will take $1 \in A_{n}$ to an element of the ideal $I$ plus an element of $Y(h)$.

The map $F(S)$ is the composition of maps $F\left(S_{k}\right): R\left(g_{k}\right) \longrightarrow R\left(g_{k+1}\right)$ corresponding to the Reidemeister moves and positive branch points. Here $1=g_{0}$, $g_{1}, \ldots, g_{m}=h$ is the sequence of braid words in some movie of $S$. Each of these maps $F\left(S_{k}\right)$ takes $1 \in A_{n} \subset R\left(g_{k}\right)$ to $\pm 1+y_{k} \in A_{n} \oplus Y\left(g_{k+1}\right)$ (this follows by a simple case-by-case verification). Therefore, $F(S)$ takes 1 to $\pm 1+y \in A_{n} \oplus Y(h)$ and cannot be null-homotopic, since \pm 1 does not lie in the ideal $I$.

This argument fails if $S$ has a negative branch point, since then the corresponding map takes 1 to $\pm\left(X_{i-1}-X_{i+1}\right)$, which does belong to $I$, plus an element of $Y\left(g_{k+1}\right)$. In fact, $F(S)=0$ if $S$ is the cobordism from the trivial braid to the braid word $\sigma_{i}^{-2}$ which creates two adjacent negative branch points, for the simple reason that 
$\left(X_{i-1}-X_{i+1}\right)^{2}=0$ in $A_{n}$. We do not know a necessary and sufficient topological condition for $F(S)$ to be zero.

An extreme version of the above positive-negative braid cobordism discrepancy is manifested in semi-trivial categorifications, sometimes the only ones available to extend a braid group action. For instance, in the categorification via Rouquier complexes, $F(S)=0$ for any cobordism with a negative branch point, but the argument in the proof of Proposition 3.7 seems to have its counterpart for Rouquier complexes, showing that $F(S) \neq 0$ for any positive braid cobordism.

A similar positive-negative imbalance exists for the invariants of braid cobordisms coming from the rings $H^{n}$ and from complexes of matrix factorizations (Examples 2.2 and 2.6 in Section 2).

Positive braid cobordisms can be realized via complex curves $[\mathbf{R u}]$ and negative cobordisms via antiholomorphic curves in $\mathbb{C}^{2}$. We believe that the positive-negative asymmetry in our examples will ultimately prove to be of the same nature as in Donaldson theory, where his invariants exhibit a markedly different behaviour on algebraic surfaces (where they are always nontrivial) and on their antiholomorphic counterparts. The same notions of positivity extend from complex to symplectic geometry, and conjecturally Donaldson's invariants never vanish on symplectic manifolds (Taubes has proved this for the Seiberg-Witten invariants). One way that positivity arises in symplectic geometry is in the monodromy of Donaldson's symplectic Lefschetz fibrations involving only positive Dehn twists. (This is directly related to the nonvanishing of Donaldson invariants, or at least Seiberg-Witten invariants, by the work of Donaldson and Smith on pseudoholomorphic multisections of such fibrations.) This manifests itself in that it was relatively easy to define positive braid moves on Fukaya-Floer categories in Section 3.4, but harder to define nontrivial representations of the negative moves.

\subsection{When Definition 3.3 fails}

There are several known and conjectural examples where a braid group (or a Coxeter braid group) acts naturally on a triangulated category $\mathcal{C}$ which decomposes nontrivially into a direct product of its indecomposable pieces, $\mathcal{C}=\underset{\mu \in J}{\oplus} \mathcal{C}_{\mu}$, for $J$ being some index set; for instance, the set of weights of an irreducible representations of a simple Lie algebra. The braid group action induces a nontrivial permutation homomorphism $\mathrm{Br}_{n} \longrightarrow S_{J}$ to the group of permutations of the set $J$. Definition 3.3 is ill-suited for such actions, since if $F_{\sigma_{i}}\left(\mathcal{C}_{\mu}\right) \subset \mathcal{C}_{\mu^{\prime}}$ for $\mu^{\prime} \neq \mu$, the only natural transformation from the identity functor on $\mathcal{C}_{\mu}$ to $F_{\sigma_{i}}$ is zero. We do not know any good substitute for Definition 3.3 which would deal with braid group actions of these type. Instead, we list several such actions below.

- The braid group action on the derived category of sheaves on disjoint unions of partial flag varieties in Section 2, Example 2.7.

- Examples 2.5 and 2.6 in Section 2 can be extended by looking at oriented tangles with "out" orientation at $k_{1}$ endpoints and "in" orientation at $k_{2}$ endpoints. The braid group on $k_{1}+k_{2}$ strands acts by braiding tangles along their endpoints. Triangulated categories then are direct sums of categories, one for each lineup of in and out orientations, and the braid group action induces a 
Homology, Homotopy and Applications, vol. 9(2), 2007

permutation action of the symmetric group $S_{k_{1}+k_{2}}$ on the set of these lineups. The braid group action extends to an invariant of oriented tangles and tangle cobordisms, and a substitute for Definition 3.3 in this case can presumably be found by restricting to a suitable subcategory of braid cobordisms.

- In a remarkable paper $[\mathbf{C R}]$, Chuang and Rouquier gave the definition and deep analysis of $\mathfrak{s l}_{2}$-categorification and, as a result of their work, introduced certain derived equivalences $\Theta$ between blocks of cyclotomic Hecke algebras, symmetric and general linear groups over finite fields, category $\mathcal{O}$, etc. It is natural to conjecture that these equivalences organize into braid group actions on direct sums of suitable blocks. In the cyclotomic Hecke algebra case these actions should categorify braid group actions on irreducible $U_{q}\left(\mathfrak{s l}_{n}\right)$-representations and affine braid group actions on integrable $U_{q}\left(\widehat{\mathfrak{s l}}_{n}\right)$ representations (if the categories are not graded, set $q=1$ ), enriching Ariki's categorification $[\mathbf{A}]$ of these representations in the $q=1$ case. Derived equivalences similar to the ones in $[\mathbf{C R}]$ should exists in derived categories of coherent sheaves on Nakajima varieties $[\mathbf{N k}]$, giving rise to Coxeter braid group actions on these categories.

\section{An affine braid group action on $D^{b}\left(T^{*} F l\right)$}

\subsection{Notation and statement of results}

Fix an $n$-dimensional complex vector space $V$, and denote by $F l$ its full flag variety

$$
F l=F l(V)=\left\{0=V_{0}<V_{1}<\cdots<V_{n-1}<V_{n}=V\right\} .
$$

Let $\pi_{i}: F l \rightarrow F l_{i}$ denote the quotient given by forgetting the $i$-th flag, so $F l_{i}$ is the variety of nested subspaces $\left(V_{j}\right)_{j \neq i}$ with $\operatorname{dim} V_{j}=j$. Fl therefore carries the tautological bundles $V_{j}$, all of them except $V_{i}$ being the pullbacks of tautological bundles (also denoted $V_{j}$ ) on $F l_{i}$. We let $L_{i}=\Lambda^{i} V_{i}^{*}$ denote the determinants of their duals.

We work on $K \stackrel{\text { def }}{=} T^{*} F l$ with the divisors $N_{i} \stackrel{\text { def }}{=} \pi_{i}^{*} T^{*} F l_{i} \subset K$ and their induced projections $p_{i}$ to $T^{*} F l_{i}$. Letting $K_{i}$ denote $T^{*} F l_{i}$, we get diagrams

$$
\begin{aligned}
& N_{i} \stackrel{\iota_{i}}{\hookrightarrow} K \\
& \downarrow^{p_{i}} \\
& K_{i} .
\end{aligned}
$$

Suppressing some obvious pullback maps for clarity, $N_{i}$ is the zero locus of the canonical section of the fiberwise cotangent sheaf $T_{\pi_{i}}^{*}=\omega_{\pi_{i}}$ given by projecting the tautological section of $T^{*} F l$ on $K=T^{*} F l$ to $T_{\pi_{i}}^{*}$. We always use $\omega$ to denote (relative) canonical bundles. Since $F l$ is the projective bundle $\mathbb{P}\left(V_{i+1} / V_{i-1}\right)$ over $F l_{i}$, we see that $T_{\pi_{i}} \cong \mathcal{H o m}\left(V_{i} / V_{i-1}, V_{i+1} / V_{i}\right)$. Thus

$$
\mathcal{O}\left(N_{i}\right) \cong \omega_{\pi_{i}} \cong \omega_{p_{i}} \cong L_{i}^{-2} \otimes L_{i-1} \otimes L_{i+1} .
$$

Define the exact functors

$$
a_{i} \stackrel{\text { def }}{=} \iota_{i *} p_{i}^{*}: D^{b}\left(K_{i}\right) \rightarrow D^{b}(K),
$$


and their right adjoints

$$
b_{i} \stackrel{\text { def }}{=} p_{i *} \iota_{i}^{!}: D^{b}(K) \rightarrow D^{b}\left(K_{i}\right) .
$$

Here $\iota_{i}^{!}=\mathcal{O}\left(N_{i}\right) \otimes \iota_{i}^{*}[-1]$ is the right adjoint of $\iota_{i *}$ by Serre duality, and is such that $\iota_{i *} \iota_{i}^{!} \cong \mathcal{H o m}_{\mathcal{O}_{K}}\left(\mathcal{O}_{N_{i}}, \cdot\right)$.

The right adjoint of $p_{i *}$ is $\omega_{p_{i}} \otimes p_{i}^{*}[1]$ by Serre duality, and the right adjoint of $\iota_{i}^{*}$ is $\iota_{i *}$, so that of $\iota_{i}^{!}$is $\iota_{i *}\left(-N_{i}\right)[1]$. Therefore the right adjoint of $b_{i}$ is

$$
\iota_{i *}\left(-N_{i}\right)[1]\left(\omega_{p_{i}} \otimes p_{i}^{*}\right)[1]=\iota_{i *} p_{i}^{*}[2]=a_{i}[2]
$$

by $(20)$.

That is we have adjunctions $a_{i} \dashv b_{i} \dashv a_{i}[2]$, and so, in particular, the counit $\mathrm{ev}_{i}: a_{i} b_{i} \rightarrow \mathrm{id}$ and unit $\mathrm{ev}_{i}^{\prime}: \operatorname{id}[-2] \rightarrow a_{i} b_{i}$. We define functors

$$
U_{i} \stackrel{\text { def }}{=} a_{i} b_{i}: D^{b}(K) \rightarrow D^{b}(K), \quad i=1, \ldots, n-1,
$$

and

$$
T_{i} \stackrel{\text { def }}{=} \operatorname{Cone}\left(U_{i} \stackrel{\mathrm{ev}_{i}}{\longrightarrow} \mathrm{id}\right), \quad T_{i}^{\prime} \stackrel{\text { def }}{=} \operatorname{Cone}\left(\mathrm{id} \stackrel{\mathrm{ev}_{i}^{\prime}}{\longrightarrow} U_{i}[2]\right)[-1] .
$$

In Section 4.4 we will prove the following.

Theorem 4.1. $T_{i} T_{i}^{\prime} \cong \mathrm{id} \cong T_{i}^{\prime} T_{i}$, and the $T_{i}$ satisfy the braid relations:

- $T_{i} T_{j} \cong T_{j} T_{i} \quad$ for $|i-j|>1$, and

- $T_{i} T_{j} T_{i} \cong T_{j} T_{i} T_{j}$ for $|i-j|=1$.

Then in Section 4.5 we will define an extra invertible functor by

$$
T \stackrel{\text { def }}{=}\left(\mathcal{L}_{1} T_{1} T_{2} \ldots T_{n-2}\right) T_{n-1}\left(T_{n-2}^{\prime} \ldots T_{2}^{\prime} T_{1}^{\prime} \mathcal{L}_{1}^{-1}\right),
$$

where $\mathcal{L}_{1}$ denotes the functor $L_{1} \otimes(\cdot)$. Calling this $T_{0}=T=T_{n}$ in a cyclic ordering, it extends the above braid group action to one of the affine braid group:

Theorem 4.2. The functor $T$ commutes with $T_{i}$ for $2 \leqslant i \leqslant n-2$, and braids with $T_{1}$ and $T_{n-1}$ :

$$
T_{0} T_{1} T_{0} \cong T_{1} T_{0} T_{1} \quad \text { and } \quad T_{n} T_{n-1} T_{n} \cong T_{n-1} T_{n} T_{n-1} .
$$

In fact we use Fourier-Mukai transforms (Section 4.2) rather than functors, giving a representation of the braid group in the group of invertible Fourier-Mukai transforms. Applying the Fourier-Mukai functor gives the above (slightly weaker) results.

\subsection{Fourier-Mukai transforms}

In places, using Fourier-Mukai kernels makes this part of the paper rather a triumph of notation over clarity. For instance, the rest of this section derives the Fourier-Mukai kernels for the above functors, and is long and formal. But it can be safely skipped: the later sections show that composition of these kernels satisfies the relations of the braid group. This will satisfy most readers, without seeing a proof that the corresponding braid group of Fourier-Mukai functors is the one described above. 
We will often suppress pullback maps applied to line bundles, and pushforwards applied to structure sheaves when the map is an embedding: given $\iota: D \hookrightarrow K$ we often denote $\iota_{*} \mathcal{O}_{D}$ by $\mathcal{O}_{\iota(D)}$ or $\mathcal{O}_{D}$ to save on notation.

If $D_{1}$ and $D_{2}$ are divisors on spaces $X_{1}$ and $X_{2}$ respectively, we use the notation $\mathcal{O}\left(D_{1}, D_{2}\right)$ for the line bundle $\mathcal{O}\left(D_{1}\right) \otimes \mathcal{O}\left(D_{2}\right)$ on any fiber product $X_{1} \times_{B} X_{2}$ of $X_{1}$ and $X_{2}$. When $X_{1}=X=X_{2}$ we let $\Delta_{X}$ (or sometimes just $\Delta$ ) denote the diagonal copy of $X$ in $X \times_{B} X$.

In several places we will have $A, B \subset X$ whose intersection $A \cap B$ is a Cartier divisor in $A$ with $\left.\mathcal{O}_{A}(A \cap B) \cong L\right|_{A}$ for some line bundle $L$ over $A \cup B$. Then we will repeatedly use the connecting homomorphism $\mathcal{O}_{B}(L) \rightarrow \mathcal{O}_{A}[1]$ of the standard exact sequence

$$
0 \rightarrow \mathcal{O}_{A} \rightarrow \mathcal{O}_{A \cup B}(L) \rightarrow \mathcal{O}_{B}(L) \rightarrow 0,
$$

i.e. the morphism representing the $\operatorname{Ext}^{1}$ class of the above extension.

$D^{b}(X)$ denotes the bounded derived category of coherent sheaves on a smooth quasi-projective variety $X$, and all functors between such categories will be the derived functors (though we omit the $\mathbf{L s}$ and $\mathbf{R s}$ ). We denote the derived dual $\mathcal{H o m}(\mathcal{F}, \mathcal{O})$ of an object $\mathcal{F}$ by $\mathcal{F}^{\vee}$.

Notoriously, cones are in general not functorial in derived categories because given an arrow one must pick a representative of its quasi-isomorphism class to take the cone on. For instance, working with the homotopy category of complexes of quasi-coherent injective sheaves with coherent cohomology, we have to pick a map of complexes in a homotopy class before taking its cone, and this choice is not functorial.

For us, however, just as in $[\mathbf{S T}]$, there is a functorial choice of map since all of our maps are evaluation (and co-evaluation) maps. Thus all of our cones will be chosen functorially.

We recommend $[\mathbf{H u}]$ for the theory of Fourier-Mukai transforms. A FourierMukai transform is an exact functor $D^{b}\left(X_{1}\right) \rightarrow D^{b}\left(X_{2}\right)$ induced by an object (Fourier-Mukai kernel) $P \in D^{b}\left(X_{1} \times X_{2}\right)$ on the product. (So, importantly, we $d o$ not insist on our Fourier-Mukai transforms being invertible.) Using the obvious projections

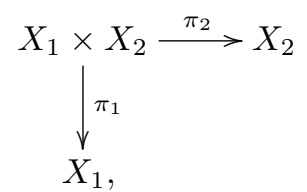

the functor is

$$
\Phi_{P} \stackrel{\text { def }}{=} \pi_{2 *}\left(P \otimes \pi_{1}^{*}(\cdot)\right) .
$$

The structure sheaf $\mathcal{O}_{\Delta}$ of the diagonal $\Delta \subset X \times X$ represents the identity on $D^{b}(X)$, and, more generally, given a morphism $f: X \rightarrow Y$, the functors $f_{*}$ and $f^{*}$ are represented by $\mathcal{O}_{(\mathrm{id} \times f) X} \in D^{b}(X \times Y)$ and $\mathcal{O}_{(f \times \mathrm{id}) X} \in D^{b}(Y \times X)$ respectively. It is a foundational result of Orlov that in fact all fully faithful exact functors arise in this way for projective $X_{i}$, though we shall not need this (our manifolds are only quasi-projective, but all the functors we use have obvious Fourier-Mukai kernels). 
However, this natural transformation $\Phi$ from $D^{b}\left(X_{1} \times X_{2}\right)$ to $\operatorname{Fun}\left(D^{b}\left(X_{1}\right), D^{b}\left(X_{2}\right)\right)$ is neither full nor faithful (see for example $[\mathbf{C a}]$ ), though it can be made to be so by working with dg-categories instead [To]. Again, this will not affect us; all of the natural transformations that we use are induced by obvious maps of kernels.

The composition of two Fourier-Mukai transforms

$$
D^{b}\left(X_{1}\right) \stackrel{\Phi_{P}}{\longrightarrow} D^{b}\left(X_{2}\right) \stackrel{\Phi_{Q}}{\longrightarrow} D^{b}\left(X_{3}\right)
$$

is $\left[\mathbf{H u}\right.$, Proposition 5.10] $\Phi_{Q \star P} \cong \Phi_{Q} \circ \Phi_{P}: D^{b}\left(X_{1}\right) \rightarrow D^{b}\left(X_{3}\right)$, where $Q \star P \in$ $D^{b}\left(X_{1} \times X_{3}\right)$ is the convolution

$$
Q \star P=\pi_{13 *}\left(\pi_{12}^{*} P \otimes \pi_{23}^{*} Q\right) .
$$

Here $\pi_{i j}$ is the projection from $X_{1} \times X_{2} \times X_{3}$ onto $X_{i} \times X_{j}$.

The right adjoint of the functor $\Phi_{P}$ is

$$
\Phi_{P^{\vee} \otimes \omega_{X_{1}}\left[-x_{1}\right]},
$$

where we are identifying $X_{1} \times X_{2}$ and $X_{2} \times X_{1}$ in the obvious way, suppressing the pullback $\pi_{1}^{*}$ on the canonical bundle $\omega_{X_{1}}$, and setting $x_{i}=\operatorname{dim} X_{i}$. By (25) the composition $\Phi_{P} \circ \Phi_{P^{\vee} \otimes \omega_{X_{1}}\left[-x_{1}\right]}$ is represented by the kernel

$$
\left(P^{\vee} \otimes \omega_{X_{1}}\left[-x_{1}\right]\right) \star P=\pi_{13 *}\left(\pi_{12}^{*} P^{\vee} \otimes \omega_{X_{1}}\left[-x_{1}\right] \otimes \pi_{23}^{*} P\right),
$$

where $\pi_{13}: X_{2} \times X_{1} \times X_{2} \rightarrow X_{2} \times X_{2}$ is the projection to the first and third factors.

Let $\delta: X_{1} \times X_{2} \hookrightarrow X_{1} \times X_{2} \times X_{1}$ be the product of $X_{1}$ 's diagonal and the identity on $X_{2}$. Then the counit $\Phi_{P} \circ \Phi_{P^{\vee} \otimes \pi_{1}^{*} \omega_{X_{1}}\left[-x_{1}\right]} \rightarrow$ id of the adjunction is represented by the morphism of Fourier-Mukai kernels given by the following composition. We restrict $(27)$ to the image of $\delta$ before applying $\pi_{13 *}$, then take the trace map and the isomorphism $\pi_{13 *} \omega_{X_{1}} \cong \mathcal{O}\left[-x_{1}\right]$ :

$$
\begin{aligned}
\pi_{13 *}\left(\pi_{12}^{*} P^{\vee} \otimes \omega_{X_{1}}\left[-x_{1}\right] \otimes \pi_{23}^{*} P\right) & \rightarrow \pi_{13 *}\left(\delta_{*}\left(P^{\vee} \otimes P\right) \otimes \omega_{X_{1}}\left[-x_{1}\right]\right) \\
& \stackrel{\operatorname{tr}}{\longrightarrow} \pi_{13 *}\left(\mathcal{O}_{\delta\left(X_{1} \times X_{2}\right)} \otimes \omega_{X_{1}}\left[-x_{1}\right]\right) \cong \mathcal{O}_{\Delta} .
\end{aligned}
$$

Using these facts we can deduce the Fourier-Mukai kernels for our functors $U_{i}$ and $T_{i}$. The reader unconcerned with the relationship to the functors $(21,22)$ could omit this and proceed to the next section where the functors are forgotten and only their Fourier-Mukai kernels $(29,32)$ are used to give a braid group representation in the group of invertible Fourier-Mukai kernels.

Lemma 4.3. The functor $U_{i}$ (21) has Fourier-Mukai kernel

$$
U_{i} \stackrel{\text { def }}{=} \mathcal{O}_{\left(\iota_{i} \times \iota_{i}\right)\left(N_{i} \times K_{i} N_{i}\right)}\left(N_{i}, 0\right)[-1] \in D^{b}(K \times K) .
$$

Proof. This is proved in a number of steps, at each stage composing Fourier-Mukai functors using (25).

- $p_{i *}: D^{b}\left(N_{i}\right) \rightarrow D^{b}\left(K_{i}\right)$ is represented by the FM kernel

$$
\mathcal{O}_{\left(\mathrm{id} \times p_{i}\right) N_{i}} \in D^{b}\left(N_{i} \times K_{i}\right) .
$$

- $p_{i}^{*}$ is represented by $\mathcal{O}_{\left(p_{i} \times \mathrm{id}\right) N_{i}} \in D^{b}\left(K_{i} \times N_{i}\right)$. 
- Therefore, the composition $p_{i}^{*} p_{i *}$ has kernel

$$
\pi_{13 *}\left[\mathcal{O}_{\left(\left(\mathrm{id} \times p_{i}\right) N_{i}\right) \times N_{i}} \otimes \mathcal{O}_{N_{i} \times\left(p_{i} \times \mathrm{id}\right) N_{i}}\right],
$$

where $\pi_{13}: N_{i} \times K_{i} \times N_{i} \rightarrow N_{i} \times N_{i}$ is the projection.

- The intersection of $\left(\left(\mathrm{id} \times p_{i}\right) N_{i}\right) \times N_{i}$ (of codimension $\operatorname{dim} K_{i}$ ) and $N_{i}$ $\times\left(p_{i} \times\right.$ id) $N_{i}$ (codimension $\left.\operatorname{dim} K_{i}\right)$ in $N_{i} \times K_{i} \times N_{i}$ is $N_{i} \times_{K_{i}} N_{i}$, which is smooth of the expected codimension $2 \operatorname{dim} K_{i}$. Therefore the (derived) tensor product of the structure sheaves equals the structure sheaf of the (transverse) intersection.

- Since $\pi_{13}$ restricted to $N_{i} \times_{K_{i}} N_{i} \subset N_{i} \times K_{i} \times N_{i}$ is an embedding, mapping it into $N_{i} \times N_{i}$ as id $\times_{K_{i}}$ id, (30) shows that the kernel for $p_{i}^{*} p_{i *}$ is $\left.\mathcal{O}_{\left(\mathrm{id} \times K_{i}\right.} \mathrm{id}\right)\left(N_{i} \times K_{K_{i}} N_{i}\right) \in D^{b}\left(N_{i} \times N_{i}\right)$.

Next we compose with $\iota_{i}^{!}$:

- $\iota_{i}^{!}=\iota_{i}^{*}\left(N_{i}\right)[-1]: D^{b}(K) \rightarrow D^{b}\left(N_{i}\right)$ is represented by the Fourier-Mukai kernel $\mathcal{O}_{\left(\iota_{i} \times \text { id }\right) N_{i}}\left(N_{i}, 0\right)[-1] \in D^{b}\left(K \times N_{i}\right)$.

- Therefore the composition $p_{i}^{*} p_{i *} !$ is represented by

$$
\left.\pi_{13 *}\left[\mathcal{O}_{\left(\left(\iota_{i} \times \mathrm{id}\right) N_{i}\right) \times N_{i}}\left(N_{i}, 0\right)[-1] \otimes \mathcal{O}_{K \times\left(\mathrm{id} \times K_{i}\right.} \mathrm{id}\right)\left(N_{i} \times K_{i} N_{i}\right)\right],
$$

where $\pi_{13}: K \times N_{i} \times N_{i} \rightarrow K \times N_{i}$ is the projection.

- The intersection of $\left(\left(\iota_{i} \times\right.\right.$ id $\left.) N_{i}\right) \times N_{i}$ and $K \times\left(\mathrm{id} \times K_{i}\right.$ id $)\left(N_{i} \times_{K_{i}} N_{i}\right)$ in $K \times N_{i} \times N_{i}$ is $N_{i} \times_{K_{i}} N_{i}$, embedded via $(a, b) \mapsto\left(\iota_{i}(a), a, b\right)$. This is smooth of the expected dimension, so the (derived) tensor product of the structure sheaves equals the structure sheaf of the (transverse) intersection.

- Composing with $\pi_{13}$ embeds $N_{i} \times_{K_{i}} N_{i}$ in $K \times N_{i}$ via $\iota_{i} \times$ id, so $p_{i}^{*} p_{i *} \iota_{i}^{!}$has kernel $\mathcal{O}_{\left(\iota_{i} \times \mathrm{id}\right)\left(N_{i} \times K_{i} N_{i}\right)}\left(N_{i}, 0\right)[-1] \in D^{b}\left(K \times N_{i}\right)$.

Finally we can compose with $\iota_{i *}$, whose kernel is $\mathcal{O}_{\left(\mathrm{id} \times \iota_{i}\right) N_{i}} \in D^{b}\left(N_{i} \times K\right)$, in the same way. The upshot is, unsurprisingly, that the Fourier-Mukai kernel for $\iota_{i *} p_{i}^{*} p_{i *} \iota_{i}^{\text {! }}$ is the pushforward of the kernel for $p_{i}^{*} p_{i *} \iota_{i}^{!}$from $D^{b}\left(K \times N_{i}\right)$ to $D^{b}(K \times K)$, i.e. $\mathcal{O}_{\left(\iota_{i} \times \iota_{i}\right)\left(N_{i} \times K_{i} N_{i}\right)}\left(N_{i}, 0\right)[-1]$, as claimed.

There is an obvious exact sequence

$$
0 \rightarrow \mathcal{O}_{\Delta} \rightarrow \mathcal{O}_{\Delta \cup\left(\left(\iota_{i} \times \iota_{i}\right)\left(N_{i} \times K_{i} N_{i}\right)\right)}\left(N_{i}, 0\right) \rightarrow \mathcal{O}_{\left(\iota_{i} \times \iota_{i}\right)\left(N_{i} \times K_{i} N_{i}\right)}\left(N_{i}, 0\right) \rightarrow 0,
$$

where $\Delta \stackrel{\iota}{\hookrightarrow} K \times K$ is the diagonal and the last arrow is the restriction map. This gives the connecting homomorphism

$$
\mathcal{O}_{\left(\iota_{i} \times \iota_{i}\right)\left(N_{i} \times_{K_{i}} N_{i}\right)}\left(N_{i}, 0\right)[-1] \rightarrow \mathcal{O}_{\Delta} .
$$

Proposition 4.4. The natural transformation $U_{i} \stackrel{\mathrm{ev}}{\longrightarrow}$ id is represented by the morphism (31) in $D^{b}(K \times K)$. Thus its cone, the functor $T_{i}$ (22), has the Fourier-Mukai kernel

$$
T_{i} \stackrel{\text { def }}{=} \mathcal{O}_{\Delta \cup\left(\iota_{i} \times \iota_{i}\right)\left(N_{i} \times K_{i} N_{i}\right)}\left(N_{i}, 0\right) \in D^{b}(K \times K) .
$$


Proof. We first claim that the counit $p_{i}^{*} p_{i *} \rightarrow$ id of the adjunction $p_{i}^{*} \dashv p_{i *}$ is the evaluation map $\mathrm{ev}_{p_{i}}$ induced by the map

$$
\mathcal{O}_{\left(\mathrm{id} \times K_{i}\right.} \text { id) }\left(N_{i} \times_{K_{i}} N_{i}\right) \rightarrow \mathcal{O}_{\Delta}
$$

given by restriction to the diagonal $\Delta \subset N_{i} \times_{K_{i}} N_{i} \subset N_{i} \times N_{i}$. This is just a notational chase and the reader might want to take it on trust, or observe that it does the right thing when applied to any object of $D^{b}\left(N_{i}\right)$.

We prove it using adjoints and the counits of (28). Since $N_{i}$ is noncompact the formulae for composition (25) and counit (28) will only work if we work relative to $K_{i}$ (notice $p_{i}: N_{i} \rightarrow K_{i}$ does have compact fibers) and use relative, instead of absolute, Serre duality. That is we replace the diagram (24) by

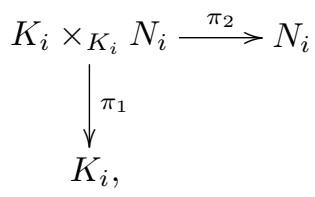

with respect to which $p_{i}^{*}$ is represented by the kernel $\mathcal{O}_{K_{i} \times_{K_{i}} N_{i}}$. Since $K_{i} \times_{K_{i}} N_{i}$ $\cong N_{i}$ and $\pi_{1}$ and $\pi_{2}$ are just $p_{i}$ and id respectively, this statement is the tautology that

$$
\pi_{2 *}\left(\mathcal{O}_{K_{i} \times_{K_{i}} N_{i}} \otimes \pi_{1}^{*}(\cdot)\right)=\mathrm{id}\left(p_{i}^{*}(\cdot)\right)=p_{i}^{*} .
$$

Then, by the relative version of $(26)$, its right adjoint $p_{i *}$ is represented by the functor

$$
\mathcal{O}_{N_{i} \times_{K_{i}} K_{i}}^{\vee} \otimes \omega_{K_{i} / K_{i}}[0]=\mathcal{O}_{N_{i} \times_{K_{i}} K_{i}},
$$

since the relative dimension of $K_{i}$ over $K_{i}$ is zero. Using, as usual, $\pi_{i j}$ to denote the projection of $N_{i} \times_{K_{i}} K_{i} \times_{K_{i}} N_{i} \rightarrow N_{i} \times_{K_{i}} N_{i}$ onto its $i$-th and $j$-th factors, by (25) the composition $p_{i}^{*} p_{i *}$ is represented by

$$
\pi_{13 *}\left(\pi_{12}^{*} \mathcal{O}_{N_{i} \times_{K_{i}} K_{i}} \otimes \pi_{23}^{*} \mathcal{O}_{K_{i} \times_{K_{i}} N_{i}}\right)=\pi_{13 *} \mathcal{O}_{N_{i} \times_{K_{i}} K_{i} \times_{K_{i}} N_{i}}=\mathcal{O}_{N_{i} \times_{K_{i}} N_{i}},
$$

and the counit $p_{i}^{*} p_{i *} \rightarrow$ id is represented by the composition (cf. (28))

$$
\pi_{13 *} \mathcal{O}_{N_{i} \times_{K_{i}} K_{i} \times_{K_{i}} N_{i}} \rightarrow \pi_{13 *}\left(\delta_{*} \mathcal{O}\right) \cong \mathcal{O}_{\Delta},
$$

where $\delta: N_{i} \times_{K_{i}} K_{i} \hookrightarrow N_{i} \times_{K_{i}} K_{i} \times_{K_{i}} N_{i}$ is the product of the diagonal on $N_{i}$ and the identity on $K_{i}$. The trace map is just the identity since we only are pushing down the dimension zero identity map $K_{i} \rightarrow K_{i}$. Thus the natural transformation $p_{i}^{*} p_{i *} \rightarrow$ id is represented by the restriction map $\mathcal{O}_{N_{i} \times_{K_{i}} N_{i}} \rightarrow \mathcal{O}_{\Delta}$. Pushing this forward from $D^{b}\left(N_{i} \times_{K_{i}} N_{i}\right)$ to $D^{b}\left(N_{i} \times N_{i}\right)$ gives (33).

Next we have to do the same for $\iota_{i *}$ and its right adjoint $\iota_{i}^{!}$. To handle the noncompactness we this time work relative to $K$, considering $N_{i}$ as a variety over $K$ (of relative dimension -1 ). So $(24)$ becomes

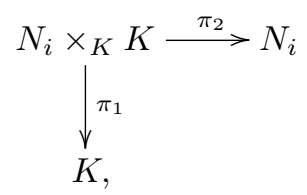


with $\iota_{i *}$ represented by the kernel $\mathcal{O}_{N_{i} \times_{K} K}$. That is $N_{i} \times_{K} K \cong N_{i}$ and $\pi_{1}, \pi_{2}$ are id, $\iota_{i}$, respectively, so

$$
\pi_{2 *}\left(\mathcal{O}_{N_{i} \times_{K} K} \otimes \pi_{1}^{*}(\cdot)\right)=\iota_{i *}(\mathrm{id})=\iota_{i *} .
$$

The Fourier-Mukai kernel for its right adjoint $\iota_{i}^{!}$is then

$$
\mathcal{O}_{N_{i} \times{ }_{K} K}^{\vee} \otimes \omega_{N_{i} / K}[-1]=\mathcal{O}_{N_{i}}\left(N_{i}\right)[-1] \in D^{b}\left(K \times_{K} N_{i}\right) .
$$

Working in $D^{b}\left(K \times_{K} N_{i} \times_{K} K\right)$ in the usual notation (25) gives the kernel for the composition $\iota_{i *} \iota_{i}^{!}$as

$$
\begin{aligned}
\pi_{13 *}\left(\pi_{12}^{*} \mathcal{O}_{N_{i}}\left(N_{i}\right)[-1] \otimes \pi_{23}^{*} \mathcal{O}_{N_{i}}\right)= & \pi_{13 *} \mathcal{O}_{N_{i}}\left(N_{i}\right)[-1] \\
& =\mathcal{O}_{N_{i}}\left(N_{i}\right)[-1] \in D^{b}\left(K \times_{K} K\right)=D^{b}(K) .
\end{aligned}
$$

(Pushing forward $D^{b}\left(K \times_{K} K\right) \rightarrow D^{b}(K \times K)$ gives its more usual absolute, rather than relative, Fourier-Mukai kernel $\mathcal{O}_{\left(\iota_{i} \times \iota_{i}\right) N_{i}}\left(N_{i}, 0\right)[-1]$.)

Finally by (28) the counit $\iota_{i *} \iota_{i}^{!} \rightarrow$ id is represented by the following morphism of Fourier-Mukai kernels. Firstly, restriction to the product (over $K$ ) of $N_{i}$ and the diagonal of $K$ in $K \times_{K} N_{i} \times_{K} K$ is the identity (since both are just $N_{i}$ ). Using the fact that $\pi_{13}$ is $\iota_{i}$, we are left with the trace map

$$
\mathcal{O}_{N_{i}}\left(N_{i}\right)[-1] \stackrel{\operatorname{tr}}{\longrightarrow} \mathcal{O}_{K}
$$

That is, we want to know what the relative Serre duality trace morphism $\omega_{N_{i} / K}[-1] \stackrel{\mathrm{tr}^{-1}}{\longrightarrow} \mathcal{O}_{K}$ is in dimension $-1=\operatorname{dim} N_{i}-\operatorname{dim} K$. (Equivalently we want to describe the natural evaluation map $\mathcal{O}_{N_{i}}^{\vee} \rightarrow \mathcal{O}_{K}$.) It is of course the connecting homomorphism of the triangle

$$
\mathcal{O}_{N_{i}}\left(N_{i}\right)[-1] \rightarrow \mathcal{O}_{K} \rightarrow \mathcal{O}_{K}\left(N_{i}\right) \rightarrow \mathcal{O}_{N_{i}}\left(N_{i}\right) .
$$

Pushing forward from $K=K \times_{K} K$ to $K \times K$ we find that $\iota_{i *} \iota_{i}^{!} \rightarrow$ id is represented by

$$
\mathcal{O}_{\left(\iota_{i} \times \iota_{i}\right) N_{i}}\left(N_{i}, 0\right)[-1] \rightarrow \mathcal{O}_{\Delta},
$$

with the morphism the pushforward of the connecting homomorphism (34) by the diagonal map $\iota_{\Delta}: \Delta \rightarrow K \times K$.

We are finally ready to describe the counit $\iota_{i *} p_{i}^{*} p_{i *} \iota_{i}^{!} \rightarrow$ id. By (33),

$$
\operatorname{ev}_{p_{i}}: \iota_{i *} p_{i}^{*} p_{i *} \iota_{i}^{!} \rightarrow \iota_{i *} \iota_{i}^{!}
$$

is represented by the restriction map

$$
\mathcal{O}_{\left(\iota_{i} \times \iota_{i}\right)\left(N_{i} \times K_{i} N_{i}\right)}\left(N_{i}, 0\right)[-1] \rightarrow \mathcal{O}_{\left(\iota_{i} \times \iota_{i}\right) N_{i}}\left(N_{i}, 0\right)[-1] .
$$

Composing with the pushforward by $\iota_{\Delta}$ of the connecting homomorphism of (34) (representing $\iota_{i *} \iota_{i}^{!} \rightarrow$ id) we obtain the counit $\iota_{i *} p_{i}^{*} p_{i *} \iota_{i}^{!} \rightarrow$ id (by the obvious composition property of units). So this is represented by the connecting morphism of 
the natural exact triangle

$$
\begin{aligned}
\mathcal{O}_{\left(\iota_{i} \times \iota_{i}\right)\left(N_{i} \times K_{i} N_{i}\right)}\left(N_{i}, 0\right)[-1] \\
\quad \rightarrow \mathcal{O}_{\Delta} \rightarrow \mathcal{O}_{\Delta \cup\left(\iota_{i} \times \iota_{i}\right)\left(N_{i} \times K_{i} N_{i}\right)}\left(N_{i}, 0\right) \rightarrow \mathcal{O}_{\left(\iota_{i} \times \iota_{i}\right)\left(N_{i} \times K_{i} N_{i}\right)}\left(N_{i}, 0\right) .
\end{aligned}
$$

Thus its cone, representing $T_{i}$, is $\mathcal{O}_{\Delta \cup\left(\left(\iota_{i} \times \iota_{i}\right)\left(N_{i} \times K_{i} N_{i}\right)\right)}\left(N_{i}, 0\right)$.

Finally, similar and equally tedious working shows that the functor id $\stackrel{\mathrm{ev}_{i}^{\prime}}{\longrightarrow} U_{i}[2]$ is represented by the morphism

$$
\mathcal{O}_{\Delta} \rightarrow \mathcal{O}_{\left(\iota_{i} \times \iota_{i}\right)\left(N_{i} \times K_{i} N_{i}\right)}\left(N_{i}, 0\right)[1]
$$

in $D^{b}(K \times K)$ which is the connecting homomorphism of the exact sequence

$$
0 \rightarrow \mathcal{O}_{\left(\iota_{i} \times \iota_{i}\right)\left(N_{i} \times K_{i} N_{i}\right)}\left(N_{i}, 0\right) \rightarrow \mathcal{O}_{\Delta \cup\left(\iota_{i} \times \iota_{i}\right)\left(N_{i} \times K_{i} N_{i}\right)}\left(L_{i}^{-1}, L_{i}\right) \rightarrow \mathcal{O}_{\Delta} \rightarrow 0,
$$

whose second arrow is the restriction to $\Delta$. We use the claim that

$$
\Delta \cap\left(\iota_{i} \times \iota_{i}\right)\left(N_{i} \times_{K_{i}} N_{i}\right)=\Delta_{N_{i}} \stackrel{\text { def }}{=}\left(\iota_{i} \times \iota_{i}\right) N_{i}
$$

is a divisor in $N_{i} \times K_{i} N_{i}$ in the class of (the restriction to $N_{i} \times K_{i} N_{i}$ of) $\left(L_{i} \otimes L_{i+1}^{-1} \otimes L_{i-1}^{-1}, L_{i}\right)$. By $(20)$, this is in turn $\left(L_{i}^{-1}\left(-N_{i}\right), L_{i}\right)$.

The claim is proved by noting that the pushdown of $\left(L_{i} \otimes L_{i+1}^{-1} \otimes L_{i-1}^{-1}, L_{i}\right)$ from $N_{i} \times K_{i} N_{i}$ to $K_{i}$ is

$$
\left(V_{i+1} / V_{i-1}\right)^{*} \otimes\left(V_{i+1} / V_{i-1}\right)^{*} \otimes \Lambda^{2}\left(V_{i+1} / V_{i-1}\right) \cong \mathcal{E} n d\left(V_{i+1} / V_{i-1}\right),
$$

since the pushdown of $\left(V_{i} / V_{i-1}\right)^{*}=L_{i} \otimes L_{i-1}^{-1}$ from $N_{i}$ to $K_{i}$ is $\left(V_{i+1} / V_{i-1}\right)^{*}$ (as everything is a pullback from the pushdown $F l \rightarrow F l_{i}$, which is the projective bundle $\mathbb{P}\left(V_{i+1} / V_{i-1}\right)$ over $\left.F l_{i}\right)$. Under this isomorphism, id $\in \mathcal{E} n d\left(V_{i+1} / V_{i-1}\right)$ gives a global section of (39) corresponding to a global section of $\left(L_{i} \otimes L_{i+1}^{-1} \otimes L_{i-1}^{-1}, L_{i}\right)$ which has zero locus the diagonal $\Delta_{N_{i}}$ (this can be checked fiberwise, where it is just the diagonal $\left.\mathbb{P}^{1} \subset \mathbb{P}^{1} \times \mathbb{P}^{1}\right)$.

Taking cones then, $T_{i}^{\prime}(22)$ has kernel

$$
\mathcal{O}_{\Delta \cup\left(\iota_{i} \times \iota_{i}\right)\left(N_{i} \times K_{i} N_{i}\right)}\left(L_{i}^{-1}, L_{i}\right) .
$$

\subsection{First results}

We now forget all about functors and work exclusively with the kernels $(29,32$, 40). To streamline notation we suppress inclusion maps when they are the obvious ones, for instance writing $\mathcal{O}_{N_{i} \times K_{i} N_{i}}\left(N_{i}, 0\right)[-1]$ for $U_{i}(29)$ and $\mathcal{O}_{\Delta \cup\left(N_{i} \times K_{i} N_{i}\right)}\left(N_{i}, 0\right)$ for $T_{i}(32)$.

Proposition 4.5. There is an exact triangle of kernels

$$
U_{i} \stackrel{a}{\longrightarrow} U_{i} \star U_{i} \stackrel{b}{\longrightarrow} U_{i}[-2]
$$

in $D^{b}(K \times K)$. It is split by either $\mathrm{id} \star \mathrm{ev}_{i}^{\prime}$ or $\mathrm{ev}_{i}^{\prime} \star \mathrm{id}: U_{i}[-2] \rightarrow U_{i} \star U_{i}$, and similarly by either id $\star \mathrm{ev}_{i}$ or $\mathrm{ev}_{i} \star \mathrm{id}: U_{i} \star U_{i} \rightarrow U_{i}$, giving isomorphisms $U_{i} \star U_{i} \cong$ $U_{i} \oplus U_{i}[-2]$. 
Remark. This is one place where using functors would have been easier and more informative. In the notation of the introduction, the natural transformation induced by $a$ is the unit id $\rightarrow b_{i} a_{i}$ of $a_{i} \dashv b_{i}$, premultiplied by $a_{i}$ and postmultiplied by $b_{i}$. A similar procedure produces $b$ from the counit $b_{i} a_{i} \rightarrow \operatorname{id}[-2]$ of $b_{i} \dashv a_{i}[2]$, and the statements about splitting then follow from standard facts about composing units and counits of adjoint functors.

Proof. In the usual notation (25),

$$
U_{i} \star U_{i}=\pi_{13 *}\left(\mathcal{O}_{N_{i} \times K_{i} N_{i} \times K}\left(N_{i}, 0,0\right)[-1] \otimes \mathcal{O}_{K \times N_{i} \times_{K_{i}} N_{i}}\left(0, N_{i}, 0\right)[-1]\right) .
$$

The intersection of the two supports is $N_{i} \times_{K_{i}} N_{i} \times_{K_{i}} N_{i}$, which is smooth but has excess intersection dimension one. Therefore the derived tensor product of the two structure sheaves has both Tor $_{0}$ (the structure sheaf of the intersection) and Tor $_{1}$. By a standard local Koszul resolution calculation the latter is given by the structure sheaf of the intersection tensored with the excess conormal bundle, which by adjunction is

$$
\begin{aligned}
& \mathcal{O}\left(-N_{i}+\omega_{K_{i}},-N_{i}, 0\right) \otimes \mathcal{O}\left(0,-N_{i},-N_{i}+\omega_{K_{i}}\right) \otimes \mathcal{O}\left(N_{i}-\omega_{K_{i}}, N_{i}, N_{i}-\omega_{K_{i}}\right) \\
& \cong \mathcal{O}\left(0,-N_{i}, 0\right) .
\end{aligned}
$$

So we have an exact triangle

$$
\begin{aligned}
\pi_{13 *} \mathcal{O}_{N_{i} \times K_{i} N_{i} \times K_{i} N_{i}}\left(N_{i}, 0,0\right)[-1] & \stackrel{a}{\longrightarrow} U_{i} \star U_{i} \\
\stackrel{b}{\longrightarrow} & \pi_{13 *} \mathcal{O}_{N_{i} \times_{K_{i}} N_{i} \times K_{i} N_{i}}\left(N_{i}, N_{i}, 0\right)[-2],
\end{aligned}
$$

where $N_{i} \times_{K_{i}} N_{i} \times_{K_{i}} N_{i}$ is embedded in $K \times K \times K$ in the obvious way via $\iota_{i} \times \iota_{i} \times \iota_{i}$. On restriction to this, $\pi_{13}$ is a $\mathbb{P}^{1}$-bundle over $N_{i} \times_{K_{i}} N_{i}$ : the pullback of $p_{i}: N_{i} \rightarrow K_{i}$ to the base $N_{i} \times_{K_{i}} N_{i}$. Thus it has relative canonical bundle the pullback of $\omega_{p_{i}} \cong \mathcal{O}\left(N_{i}\right)$, which is $\mathcal{O}\left(0, N_{i}, 0\right)$. Since $p_{i *} \mathcal{O} \cong \mathcal{O}$ and $p_{i *} \omega_{p_{i}} \cong \mathcal{O}[-1]$ by Serre duality, (43) becomes

$$
\mathcal{O}_{\left(\iota_{i} \times \iota_{i}\right)\left(N_{i} \times K_{i} N_{i}\right)}\left(N_{i}, 0\right)[-1] \rightarrow U_{i} \star U_{i} \rightarrow \mathcal{O}_{\left(\iota_{i} \times \iota_{i}\right)\left(N_{i} \times K_{i} N_{i}\right)}\left(N_{i}, 0\right)[-3],
$$

which by (29) gives (41).

The morphism $1 \star \mathrm{ev}_{i}^{\prime}: U_{i}[-2] \rightarrow U_{i}^{2}$ in $D^{b}(K \times K)$ is

$$
\begin{aligned}
\pi_{13 *}\left(\mathcal{O}_{N_{i} \times K_{i}} N_{i} \times K\right. & \left(N_{i}, 0,0\right)[-1] \otimes \mathcal{O}_{K \times \Delta}[-2] \\
& \left.\longrightarrow \mathcal{O}_{N_{i} \times_{K_{i}} N_{i} \times K}\left(N_{i}, 0,0\right)[-1] \otimes \mathcal{O}_{K \times N_{i} \times_{K_{i}} N_{i}}\left(0, N_{i}, 0\right)[-1]\right),
\end{aligned}
$$

where the map is the identity on the left-hand sides of the tensor products and $\mathrm{ev}_{i}^{\prime}$ of (37) on the right-hand sides.

We wish to show that the composition of this map with $U_{i} \star U_{i} \stackrel{b}{\rightarrow} U_{i}[-2]$ is the identity. But $b$ was defined by projecting the second derived tensor product in (45) 
to its degree zero $\left(\mathrm{Tor}_{0}\right)$ part. That is, the composition is given by

$$
\pi_{13 *}\left(\mathcal{O}_{N_{i} \times K_{i} \Delta_{N_{i}}}\left(N_{i}, 0,0\right)[-3] \rightarrow \mathcal{O}_{N_{i} \times_{K_{i}} N_{i} \times K_{i} N_{i}}\left(N_{i}, N_{i}, 0\right)[-2]\right),
$$

where the map is the connecting homomorphism of the natural divisor exact sequence

$$
0 \rightarrow \mathcal{O}\left(N_{i}, N_{i}, 0\right) \rightarrow \mathcal{O}\left(N_{i}, N_{i}, 0\right)\left(N_{i} \times_{K_{i}} \Delta_{N_{i}}\right) \rightarrow \mathcal{O}_{N_{i} \times_{K_{i}} \Delta_{N_{i}}}\left(N_{i}, 0,0\right) \rightarrow 0,
$$

(cf. (38)) pushed forward from $N_{i} \times{ }_{K_{i}} N_{i} \times{ }_{K_{i}} N_{i}$ to $K \times K \times K$ and shifted by $[-3]$.

So the cone on $(46)$ is

$$
\pi_{13 *}\left(\mathcal{O}_{N_{i} \times K_{i}} N_{i} \times_{K_{i}} N_{i}\left(N_{i}, N_{i}, 0\right)\left(N_{i} \times_{K_{i}} \Delta_{N_{i}}\right)[-2]\right) \cong 0,
$$

because $\mathcal{O}\left(N_{i}, N_{i}, 0\right)\left(N_{i} \times K_{i} \Delta_{N_{i}}\right)$ is a line bundle of degree -1 on the $\mathbb{P}^{1}$-fibers of $\pi_{13} \mid N_{i} \times_{K_{i}} N_{i} \times_{K_{i}} N_{i}$.

Thus the induced map in (46),

$$
\mathcal{O}_{N_{i} \times_{K_{i}} N_{i}}\left(N_{i}, 0\right)[-3] \rightarrow \mathcal{O}_{N_{i} \times_{K_{i}} N_{i}}\left(N_{i}, 0\right)[-3],
$$

must be a nonzero multiple of the identity, and so isomorphic to the identity, as required.

The proof that $\mathrm{ev}_{i}^{\prime} \star 1$ also splits (41) is very similar.

To analyse $\mathrm{ev}_{i} \star 1: U_{i} \star U_{i} \rightarrow U_{i}$ we tensor the following exact sequence (the product of (36) with $K)$ with $\mathcal{O}_{K \times N_{i} \times K_{i} N_{i}}\left(0, N_{i}, 0\right)$ :

$$
0 \rightarrow \mathcal{O}_{\Delta \times K} \rightarrow \mathcal{O}_{\left(\Delta \cup\left(N_{i} \times K_{i} N_{i}\right)\right) \times K}\left(N_{i}, 0,0\right) \rightarrow \mathcal{O}_{N_{i} \times_{K_{i}} N_{i} \times K}\left(N_{i}, 0,0\right) \rightarrow 0 .
$$

Taking $\pi_{13 *}$ of the connecting homomorphism and shifting by $[-2]$ gives ev $_{i} \star 1$. Now the intersection of $K \times N_{i} \times K_{i} N_{i}$ with both of $\left(\Delta \cup\left(N_{i} \times K_{i} N_{i}\right)\right) \times K$ and $N_{i} \times K_{i} N_{i} \times K$ is the same $\left(N_{i} \times K_{i} N_{i} \times K_{i} N_{i}\right)$, so in the long exact sequence of Tors the final two terms are the same with the restriction map between them an isomorphism. Thus the next two terms to the left give the exact sequence

$$
\operatorname{Tor}_{1}\left(\mathcal{O}_{N_{i} \times K_{i}} N_{i} \times K, \mathcal{O}_{K \times N_{i} \times K_{i} N_{i}}\right)\left(N_{i}, N_{i}, 0\right) \rightarrow \mathcal{O}_{\Delta_{N_{i}} \times_{K_{i}} N_{i}}\left(N_{i}, 0,0\right) \rightarrow 0
$$

Now recall (43) that the inclusion of this Tor $_{1}$ term into the full derived tensor product is precisely what gives the map $a: U_{i} \rightarrow U_{i} \star U_{i}$; i.e. applying $\pi_{13 *}[-1]$ to the above two terms gives $\left(\operatorname{ev}_{i} \star 1\right) \circ a$. We also computed this $\operatorname{Tor}_{1}$ in $(42)$; the upshot is that $\left(\mathrm{ev}_{i} \star 1\right) \circ a$ is

$$
\pi_{13 *}\left[\mathcal{O}_{N_{i} \times_{K_{i}} N_{i} \times_{K_{i}} N_{i}} \rightarrow \mathcal{O}_{\Delta_{N_{i}} \times_{K_{i}} N_{i}}\right]\left(N_{i}, 0\right)[-1],
$$

with the map inside the brackets the surjection of structure sheaves. Thus this map is isomorphic to the restriction map; when we apply $\pi_{13 *}$ we get

$$
\left[\mathcal{O}_{N_{i} \times K_{i} N_{i}} \rightarrow \mathcal{O}_{N_{i} \times K_{i}} N_{i}\right]\left(N_{i}, 0\right)[-1]
$$

on $K \times K$, with the map the identity. So $\left(\mathrm{ev}_{i} \star 1\right) \circ a$ is isomorphic to the identity map $U_{i} \rightarrow U_{i}$, and $\mathrm{ev}_{i} \star 1$ splits (41). The proof for $1 \star \mathrm{ev}_{i}$ is very similar.

We are now ready to prove invertibility of our functors. This can by now be proved in many ways. It follows as a special case of the results of Horja [Ho] or 
similar ideas of Szendröi $[\mathbf{S z 1}]$, for instance, and the quickest proof is probably by adapting Ploog's method $[\mathbf{P l}, \mathbf{H u}]$ for the invertibility of the spherical twists $[\mathbf{S T}]$ to this family situation. We give a proof inspired by purely categorical methods and the earlier invertibility proofs in $[\mathbf{K S}, \mathbf{S T}]$ and the more general result proved by Rouquier [Ro1], at the level of Fourier-Mukai transforms.

Proposition 4.6. In $D^{b}(K \times K)$ we have quasi-isomorphisms $T_{i} \star T_{i}^{\prime} \cong \mathcal{O}_{\Delta}$ $\cong T_{i}^{\prime} \star T_{i}$.

Proof. $T_{i}^{\prime} \star T_{i}$ is the total complex of

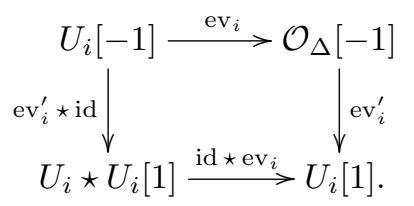

We map the trivial cone $U_{i}[1] \stackrel{\text { id }}{\rightarrow} U_{i}[1]$ into the bottom row of (47) by the map $a[1]$ on the first factor and the identity on the second. By Proposition 4.5 this is indeed a morphism in $D^{b}(K \times K)$ and taking the cone makes $T_{i}^{\prime} \star T_{i}$ isomorphic to the total complex of

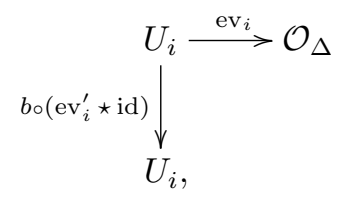

where now the first $U_{i}$ is in degree 1 and the other two terms are in degree 0 . By Proposition 4.5 the vertical arrow is isomorphic to the identity, so $T_{i}^{\prime} \star T_{i} \cong \mathcal{O}_{\Delta}$. The proof that $T_{i} \star T_{i}^{\prime} \cong \mathcal{O}_{\Delta}$ is very similar.

Defining $F l_{i j}, i \neq j$, to be the quotient of $F l$ given by forgetting both the $i$-th and $j$-th subspaces, we have natural maps

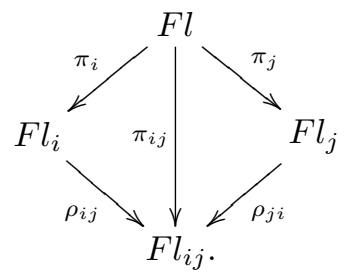

$\pi_{i}$ and $\pi_{j}$ are $\mathbb{P}^{1}$-bundles, and if $|i-j|>1$ then $\rho_{i j}$ and $\rho_{j i}$ are also; in this case the diagram is Cartesian, i.e. $\pi_{i j}$ is the fiber product of $\rho_{i j}$ and $\rho_{j i}$. (For $|i-j|=1$, $\rho_{i j}$ and $\rho_{j i}$ are $\mathbb{P}^{2}$-bundles.)

We also define $N_{i j}$ to be the (transverse) intersection $N_{i} \cap N_{j}$ inside $K$. For $|i-j|>1$ this is the pullback $\pi_{i j}^{*} T^{*} F l_{i j}$. Finally we set $K_{i j} \stackrel{\text { def }}{=} \rho_{i j}^{*} T^{*} F l_{i j}$, a divisor in $K_{i}=T^{*} F l_{i}$, the zero set of the canonical section of $T^{*} \rho_{i j}$. It is the image of $N_{i j}$ under $p_{i}: N_{i} \rightarrow K_{i}=T^{*} F l_{i}$.

With this notation we can prove the following. 
Proposition 4.7. For $|i-j|>1$ we have $T_{i} \star T_{j} \cong T_{j} \star T_{i} \in D^{b}(K \times K)$.

Proof. In the usual notation we calculate $T_{i} \star T_{j}$ as

$$
\pi_{13 *}\left(\mathcal{O}_{K \times\left(\Delta \cup\left(N_{i} \times_{K_{i}} N_{i}\right)\right)}\left(0, N_{i}, 0\right) \otimes \mathcal{O}_{\left(\Delta \cup\left(N_{j} \times K_{j} N_{j}\right)\right) \times K}\left(N_{j}, 0,0\right)\right) .
$$

Both supports have codimension $\operatorname{dim} K$ in $K \times K \times K$, and their intersection is

$$
(\mathrm{id} \times \mathrm{id} \times \mathrm{id}) K \cup\left(\Delta_{N_{i}} \times_{K_{i}} N_{i}\right) \cup\left(N_{j} \times_{K_{j}} \Delta_{N_{j}}\right) \cup\left(N_{j} \times_{K_{j}} N_{i j} \times_{K_{i}} N_{i}\right) .
$$

The last component $N_{j} \times_{K_{j}} N_{i j} \times_{K_{i}} N_{i}$ is $N_{i j} \times_{K_{j i}} N_{i j} \times_{K_{i j}} N_{i j}$, which in turn equals $N_{i j} \times_{T^{*} F l_{i j}} N_{i j}$ by pulling back $T^{*} F l_{i j}$ to both sides of the isomorphism $F l \times{ }_{F l_{j}} F l \times \times_{F l_{i}} F l \cong F l \times{ }_{F l_{i j}} F l$ (which amounts to the statement that (48) is Cartesian).

Therefore all four irreducible components are of codimension $2 \operatorname{dim} K$, the intersection is a local complete intersection and the derived tensor product of the structure sheaves is just the structure sheaf of the intersection. Moreover, $\pi_{13}$ restricted to each component is an embedding into $K \times K$, yielding

$$
T_{i} \star T_{j} \cong \mathcal{O}_{\Delta \cup\left(N_{i} \times_{K_{i}} N_{i}\right) \cup\left(N_{j} \times K_{j} N_{j}\right) \cup\left(N_{i j} \times_{T^{*} F l_{i j}} N_{i j}\right)}\left(D_{i j}\right) .
$$

We claim that $\mathcal{O}\left(D_{i j}\right)$ is $\mathcal{O}\left(N_{i}+N_{j}, 0\right)$, i.e. that $\mathcal{O}\left(0, N_{i}, 0\right)$ in (49) is isomorphic to $\mathcal{O}\left(N_{i}, 0,0\right)$ on restriction to the intersection. This is obvious on the first two irreducible components of the intersection, and on the latter two it follows from the fact that $\mathcal{O}_{N_{j}}\left(N_{i}\right)$ is pulled back from $K_{j}$ (for $|i-j|>1$ : it is the pullback of $\left.\mathcal{O}_{K_{j}}\left(K_{j i}\right)\right)$.

So $D_{i j}$ and therefore also (50) are symmetric in $i$ and $j$.

\subsection{The Yang-Baxter equation}

In this section we fix $j=i+1$ and prove the final braid relation $T_{i} \star T_{j} \star T_{i}$ $\cong T_{j} \star T_{i} \star T_{j}$.

The geometry and notation become especially nasty in this section, and the interested reader is advised to set $n=3$ and $i, j=1,2$, so that $F l=F l\left(\mathbb{C}^{3}\right)$ fibers by $\pi_{2}$ over $F l_{2}=\mathbb{P}^{2}$ and by $\pi_{1}$ over $F l_{1}=\mathbb{P}^{2 *}$. In this case $M_{i j}$ below is just the zero section $F l \subset T^{*} F l$, while $K_{i j}=\mathbb{P}^{2} \subset T^{*} \mathbb{P}^{2}=K_{2}$ and $K_{j i}=\mathbb{P}^{2 *} \subset T^{*} \mathbb{P}^{2 *}$ $=K_{1}$. Lemma 4.8 is then just the Mukai flop. The geometry that results in this special case is where all the action takes place in the general case (which is a bundle over $T^{*} F l_{i j}$ with fiber the $n=3$ geometry).

We refer to the diagram (48). As before we define $N_{i j}$ to be the (transverse) intersection $N_{i} \cap N_{j}$ inside $K$, and we set $M_{i j} \stackrel{\text { def }}{=} \pi_{i j}^{*} T^{*} F l_{i j}$. Since $|i-j|=1$ these are not equal, but $M_{i j} \subset N_{i j}$ is a divisor, in fact an exceptional divisor:

Lemma 4.8. The map $\left.q_{i} \stackrel{\text { def }}{=} p_{i}\right|_{N_{i j}}$ is the blow-up of $K_{i}=T^{*} F l_{i}$ in

$$
K_{i j} \stackrel{\text { def }}{=} \rho_{i j}^{*} T^{*} F l_{i j}
$$

and $M_{i j}$ is the exceptional divisor.

Symmetrically, $\left.q_{j} \stackrel{\text { def }}{=} p_{j}\right|_{N_{i j}}$ is the blow-up of $K_{j}=T^{*} F l_{j}$ in $K_{j i}=\rho_{j i}^{*} T^{*} F l_{i j}$ with the same exceptional divisor $M_{i j} \subset N_{i j}$. 
Proof. Thinking of $N_{i j}$ as the zero set of the canonical section of $T^{*} \pi_{j}$ restricted to $N_{i}=\pi_{i}^{*} T^{*} F l_{i}$, we see that the functions that are linear on the fibers of its projection to $F l$ are

$$
\frac{\pi_{i}^{*} T F l_{i}}{T \pi_{j}}
$$

These generate the ideal of the zero section $F l$; the subset of those that vanish on the divisor $M_{i j}=\pi_{i j}^{*} T^{*} F l_{i j} \subset N_{i j}$ is

$$
\begin{aligned}
\operatorname{ker}\left(\frac{\pi_{i}^{*} T F l_{i}}{T \pi_{j}} \longrightarrow \pi_{i j}^{*} T F l_{i j}\right) & =\frac{\pi_{i}^{*} T \rho_{i j}}{T \pi_{j}}=\frac{\mathcal{H o m}\left(V_{j} / V_{i-1}, V_{j+1} / V_{j}\right)}{\mathcal{H o m}\left(V_{j} / V_{i}, V_{j+1} / V_{j}\right)} \\
& =\mathcal{H o m}\left(\frac{V_{i}}{V_{i-1}}, \frac{V_{j+1}}{V_{j}}\right)=\left(\frac{V_{i}}{V_{i-1}}\right)^{*} \otimes\left(\frac{V_{j+1}}{V_{j}}\right) .
\end{aligned}
$$

Thinking of $\pi_{i}: F l \rightarrow F l_{i}$ as the projective bundle $\mathbb{P}\left(V_{j} / V_{i-1}\right) \rightarrow F l_{i}$ with tautological line bundle $\mathcal{O}_{\mathbb{P}}(-1)=V_{i} / V_{i-1}$, we see that the pushdown of $\left(V_{i} / V_{i-1}\right)^{*}$ $=\mathcal{O}_{\mathbb{P}}(1)$ is $\left(V_{j} / V_{i-1}\right)^{*}$. Therefore, the pushdown of the above ideal is

$$
\left(\frac{V_{j}}{V_{i-1}}\right)^{*} \otimes\left(\frac{V_{j+1}}{V_{j}}\right)=\mathcal{H o m}\left(\frac{V_{j}}{V_{i-1}}, \frac{V_{j+1}}{V_{j}}\right)=T \rho_{i j} .
$$

But this is the subset of the linear functions $T F l_{i}$ on $K_{i}=T^{*} F l_{i}$ that vanish on $\rho_{i j}^{*} T^{*} F l_{i j}=K_{i j}$. Thus we have shown that

$$
q_{i *}\left(\mathcal{O}_{N_{i j}}\left(-M_{i j}\right)\right)=\mathcal{I}_{K_{i j} \subset K_{i}} .
$$

Since everything in sight is smooth, this is the defining relation of the blow-up of $K_{i}$ in $K_{i j}$.

We will also need the following.

Lemma 4.9. The natural projection $M_{i j} \times_{K_{j i}} M_{i j} \rightarrow K_{i j} \times_{T^{*} F l_{i j}} K_{i j}$ is the blowup of $K_{i j} \times_{T^{*} F l_{i j}} K_{i j}$ in the diagonal $K_{i j}$.

Proof. This is proved by pulling back $T^{*} F l_{i j}$ to

$$
F l \times_{F l_{j}} F l \cong \mathrm{Bl}_{\Delta_{F l_{i}}}\left(F l_{i} \times_{F l_{i j}} F l_{i}\right) .
$$

To prove (51) we exhibit a rational map $F l_{i} \times_{F l_{i j}} F l_{i}-\rightarrow F l_{j}$ that blows up the diagonal $\Delta_{F l_{i}}$. This map takes the $V_{j}$ subspaces of $V$ in the two copies of $F l_{i}$ and intersects them to define the $V_{i}$ subspace of $V$ in $F l_{j}$, and so is ill-defined over the diagonal where this intersection is too big.

We define the map through sections of line bundles, and work relative to $F l_{i j}$. Think of $F l_{j} \rightarrow F l_{i j}$ as the projective bundle $\mathbb{P}\left(V_{j+1} / V_{i-1}\right)$ with its tautological line bundle $\mathcal{O}_{\mathbb{P}}(-1)=V_{i} / V_{i-1}$, and work with the line bundle $\Lambda^{3}\left(V_{j+1} / V_{i-1}\right) \otimes \mathcal{O}_{\mathbb{P}}(1)$ on $F l_{j}$. Its pushdown to $F l_{i j}$ is

$$
\Lambda^{3}\left(V_{j+1} / V_{i-1}\right) \otimes\left(V_{j+1} / V_{i-1}\right)^{*} \cong \Lambda^{2}\left(V_{j+1} / V_{i-1}\right) .
$$

Similarly we think of $F l_{i} \rightarrow F l_{i j}$ as the projective bundle $\mathbb{P}\left(\left(V_{j+1} / V_{i-1}\right)^{*}\right)$ with tautological line bundle $\mathcal{O}_{\mathbb{P}}(-1)=\left(V_{j+1} / V_{j}\right)^{*}$. The pushdown on $\mathcal{O}_{\mathbb{P}}(1)$ is then $V_{j+1} / V_{i-1}$. 
Homology, Homotopy and Applications, vol. 9(2), 2007

Thus the sections of $\mathcal{O}(1,1)$ on $F l_{i} \times_{F l_{i j}} F l_{i}$ over $F l_{i j}$ are

$$
\left(V_{j+1} / V_{i-1}\right) \otimes\left(V_{j+1} / V_{i-1}\right),
$$

containing the sections (52) as the antisymmetric tensors. These latter sections vanish precisely (scheme theoretically) on the diagonal $\Delta_{F l_{i}}$ and generate its ideal, so we get the blow-up claimed.

The resulting map $\mathrm{Bl}_{\Delta_{F l_{i}}}\left(F l_{i} \times_{F l_{i j}} F l_{i}\right) \rightarrow F l_{j}$ is the projection

$$
\mathrm{Fl} \times{ }_{F l_{j}} \mathrm{Fl} \rightarrow \mathrm{Fl}_{j}
$$

since a flag is determined by its projections to $F l_{i}$ and $F l_{j}$ (i.e. $\pi_{i} \times \pi_{j}$ : $F l \rightarrow F l_{i} \times F l_{j}$ is an embedding).

We compute $U_{i} \star U_{j}$ in the usual way (25):

$$
U_{i} \star U_{j}=\pi_{13 *}\left(\mathcal{O}_{N_{j} \times_{K_{j}} N_{j} \times K}\left(N_{j}, 0,0\right)[-1] \otimes \mathcal{O}_{K \times N_{i} \times K_{i} N_{i}}\left(0, N_{i}, 0\right)[-1]\right) .
$$

Both supports have codimension in $K \times K \times K$ equal to $\operatorname{dim} K$, and their intersection

$$
N_{j} \times_{K_{j}} N_{i j} \times_{K_{i}} N_{i} \subset K \times K \times K
$$

is smooth (being a $\mathbb{P}^{1} \times \mathbb{P}^{1}$-bundle over $N_{i j}$ ) of codimension $2 \operatorname{dim} K$. Thus the intersection is transverse, and

$$
U_{i} \star U_{j}=\pi_{13 *}\left(\mathcal{O}_{N_{j} \times_{K_{j}} N_{i j} \times_{K_{i}} N_{i}}\left(N_{j}, N_{i}, 0\right)\right)[-2] .
$$

On restriction to $(53), \pi_{13}$ maps $(a, b, c)$ to $\left(\iota_{i}(a), \iota_{j}(c)\right) \in K \times K$. A point of the image determines $a$ and $c$ (since $\iota_{i}$ and $\iota_{j}$ are injections) and so $q_{j}(b)$ and $q_{i}(b)$ as $p_{j}(a)$ and $p_{i}(c)$ respectively. But this determines $b$ completely and so the map is injective. In fact it is an embedding because $\iota_{i}, \iota_{j}$ and $q_{i} \times q_{j}: N_{i j} \hookrightarrow K_{i} \times K_{j}$ are (the latter because $\pi_{i} \times \pi_{j}: F l \hookrightarrow F l_{i} \times F l_{j}$ is). Therefore

$$
U_{i} \star U_{j}=\mathcal{O}_{N_{j} \times K_{j}} N_{i j} \times_{K_{i}} N_{i}(D)[-2] \in D^{b}(K \times K),
$$

where $N_{j} \times_{K_{j}} N_{i j} \times_{K_{i}} N_{i}$ is embedded into $K \times K$ as described above, and $D$ is the divisor thereon given by restricting $\mathcal{O}\left(N_{j}, N_{i}, 0\right)$ from $K \times K \times K$ via the inclusion (53).

Recall the description of the morphism ev $\mathrm{e}_{i}$ as a connecting homomorphism (31). Chasing this through the above derivation of $U_{i} \star U_{j}$ shows that id $\star \mathrm{ev}_{j}: U_{i} \star U_{j}$ $\rightarrow U_{i}$ is given by (the shift by [-2] of) the connecting homomorphism of the exact sequence

$$
\begin{aligned}
& 0 \rightarrow \mathcal{O}_{N_{i} \times K_{i} N_{i}}\left(N_{i}, 0\right) \rightarrow \mathcal{O}_{\left(N_{i} \times K_{i} N_{i}\right) \cup_{N_{i j} \times K_{i}} N_{i}}\left(N_{j} \times{ }_{K_{j}} N_{i j} \times K_{i} N_{i}\right) \\
& \rightarrow \mathcal{O}_{N_{j} \times K_{j}} N_{i j} \times_{K_{i}} N_{i} \\
&(D) \rightarrow 0
\end{aligned}
$$

that arises from the fact that $N_{i} \times_{K_{i}} N_{i}$ and $N_{j} \times_{K_{j}} N_{i j} \times_{K_{i}} N_{i}$ intersect in $N_{i j} \times_{K_{i}} N_{i}$, which is a divisor in both. Here $\mathcal{O}(D)$ is the line bundle on the union which is $\mathcal{O}_{N_{i} \times_{K_{i}} N_{i}}\left(N_{i}+N_{j}, 0\right)$ glued to $\mathcal{O}_{N_{j} \times K_{j}} N_{i j} \times_{K_{i}} N_{i}(D)$ across $N_{i j} \times_{K_{i}} N_{i}$ by their common restriction to $\mathcal{O}_{N_{i j} \times K_{i} N_{i}}\left(L_{i}^{-2}+L_{i-1}+N_{j}, L_{j}\right)$ (by (20) and the fact 
that $L_{j}$ is pulled back from a line bundle $L_{j}$ on $K_{i}$ and so can be dragged across the first fiber product).

We introduce one more piece of notation. Just as the kernel $U_{i}$ represents a functor $\iota_{i *} p_{i}^{*} p_{i *} \iota_{i}^{!}$arising naturally from the diagram (19), the diagram

$$
\begin{gathered}
M_{i j} \stackrel{\iota_{i j}}{\hookrightarrow} K \\
p_{i j} \\
T^{*} F l_{i j}
\end{gathered}
$$

induces the functor $\iota_{i j *} p_{i j}^{*} p_{i j *} \iota_{i j}^{!}$on $D^{b}(K)$. By much the same working as in Proposition 4.3 this can be shown to be represented by the kernel

$$
U_{i j} \stackrel{\text { def }}{=} \mathcal{O}_{M_{i j} \times_{T^{*} F l_{i j}} M_{i j}}\left(\omega_{F l / F l_{i j}}, 0\right)[-3] \in D^{b}(K \times K) .
$$

Proposition 4.10. There is an isomorphism $U_{i} \star U_{j} \star U_{i} \cong U_{i}[-2] \oplus U_{i j}$, such that the composition of $U_{i} \star U_{j} \star U_{i} \stackrel{\mathrm{ev}_{j}}{\longrightarrow} U_{i} \star U_{i}$ and the map $U_{i} \star U_{i} \stackrel{b}{\longrightarrow} U_{i}[-2]$ of Proposition 4.5 acts as the identity on $U_{i}[-2]$.

Proof. By (54) and (25), in the notation above $U_{i} \star U_{j} \star U_{i}$ is

$$
\pi_{13 *}\left(\mathcal{O}_{N_{i} \times K_{i} N_{i} \times K}\left(N_{i}, 0,0\right)[-1] \otimes \mathcal{O}_{K \times\left(N_{j} \times K_{j} N_{i j} \times K_{i} N_{i}\right)}(0, D)[-2]\right) .
$$

Both supports have codimension $\operatorname{dim} K$ in $K \times K \times K$. Their intersection

$$
\begin{aligned}
N_{i} \times_{K_{i}} N_{i j} \times_{K_{j}} & N_{i j} \times_{K_{i}} N_{i} \subset K \times K \times K, \\
(a, b, d, c) & \mapsto(a, b, c),
\end{aligned}
$$

has $b=d$ except over the exceptional locus $M_{i j} \times_{K_{j}} M_{i j} \subset N_{i j} \times_{K_{j}} N_{i j}$ where the projections $q_{i}: N_{i j} \rightarrow K_{i}$ and $q_{j}: N_{i j} \rightarrow K_{j}$ are not isomorphisms. Therefore (56) is the union $A \cup B$ of two irreducible components; the first where $b=d$,

$$
A=N_{i} \times_{K_{i}} N_{i j} \times_{K_{i}} N_{i}
$$

the second the closure of the locus where $b \neq d$,

$$
B=M_{i j} \times_{K_{i j}} M_{i j} \times_{K_{j i}} M_{i j} \times_{K_{i j}} M_{i j}
$$

These both have codimension $2 \operatorname{dim} K$, so that the derived tensor product of the structure sheaves is the structure sheaf of $A \cup B$, and

$$
U_{i} \star U_{j} \star U_{i} \cong \pi_{13 *}\left(\mathcal{O}_{A \cup B}\left(N_{i}, 0,0\right)(0, D)\right)[-3]
$$

Set $E \stackrel{\text { def }}{=} A \cap B=M_{i j} \times_{K_{i j}} M_{i j} \times_{K_{j i}} M_{i j}$, a divisor in both $A$ and $B$. We will show that the inclusion of the natural subsheaf $\mathcal{O}_{A}(-E) \oplus \mathcal{O}_{B}(-E) \hookrightarrow \mathcal{O}_{A \cup B}$ induces an isomorphism

$$
\pi_{13 *}\left(\mathcal{O}_{A}\left(N_{i}, 0,0\right)(0, D)(-E)\right) \oplus \pi_{13 *}\left(\mathcal{O}_{B}\left(N_{i}, 0,0\right)(0, D)(-E)\right)
$$


Homology, Homotopy and Applications, vol. 9(2), 2007

$$
\stackrel{\simeq}{\longrightarrow} \pi_{13 *}\left(\mathcal{O}_{A \cup B}\left(N_{i}, 0,0\right)(0, D)\right)
$$

It is therefore sufficient to show that

$$
\pi_{13 *}\left(\mathcal{O}_{A}\left(N_{i}, 0,0\right)(0, D)(-E)\right) \rightarrow \pi_{13 *}\left(\mathcal{O}_{A}\left(N_{i}, 0,0\right)(0, D)\right)
$$

and

$$
\pi_{13 *}\left(\mathcal{O}_{B}\left(N_{i}, 0,0\right)(0, D)(-E)\right) \rightarrow \pi_{13 *}\left(\mathcal{O}_{B}\left(N_{i}, 0,0\right)(0, D)\right)
$$

are isomorphisms.

On restriction to $A, \pi_{13}$ projects out the $b$-variable to $N_{i} \times_{K_{i}} N_{i}$. As such it is the pullback via $N_{i} \times_{K_{i}} N_{i} \rightarrow K_{i}$ of the map $q_{i}: N_{i j} \rightarrow K_{i}$ (i.e. the basechange of $q_{i}$ from $K_{i}$ to $N_{i} \times_{K_{i}} N_{i}$ ).

By Lemma $4.8 q_{i}$ is the blow-up of $K_{i}$ in $K_{i j}$ of codimension two, so $\omega_{q_{i}}$ is the exceptional divisor $\mathcal{O}_{N_{i j}}\left(M_{i j}\right)$. Pulling back to $A$ we get $\mathcal{O}_{A}(E)$, where $E$ is the exceptional divisor of $\left.\pi_{13}\right|_{A}$; i.e. $E=M_{i j} \times_{K_{i j}} M_{i j} \times_{K_{i j}} M_{i j}=A \cap B$.

Since $\omega_{K_{i}} \cong \mathcal{O}_{K_{i}}$, this relative canonical bundle is also $\omega_{N_{i j}}$, which by adjunction is $\left.\mathcal{O}\left(N_{i}+N_{j}\right)\right|_{N_{i j}}$, i.e. $\mathcal{O}\left(0, N_{1}+N_{j}, 0\right)$ on pullback to $A$. But since $b=d$ on $A$ we also have $\left.\mathcal{O}(0, D)\right|_{A}=\mathcal{O}\left(0, N_{i}+N_{j}, 0\right)$ from its definition (54). The upshot is that

$$
\left.\mathcal{O}_{N_{i j}}\left(M_{i j}\right) \cong \mathcal{O}\left(N_{i}+N_{j}\right)\right|_{N_{i j}} \text { and } \mathcal{O}_{A}(0, D)=\mathcal{O}_{A}(E) \text {. }
$$

It follows that $(60)$ is $\pi_{13 *}\left(\mathcal{O}_{A}\left(N_{i}, 0,0\right)\right) \rightarrow \pi_{13 *}\left(\mathcal{O}_{A}\left(N_{i}, 0,0\right)(E)\right)$. Since $E$ is exceptional this is an isomorphism $\mathcal{O}_{N_{i} \times K_{i} N_{i}}\left(N_{i}, 0\right) \stackrel{\simeq}{\longrightarrow} \mathcal{O}_{N_{i} \times K_{i}} N_{i}\left(N_{i}, 0\right)$ as required.

Something similar happens for $B$. The map $\left.\pi\right|_{B}$ projects $B$ to $M_{i j} \times_{T^{*} F l_{i j}} M_{i j} \subset$ $K \times K$, contracting only the exceptional divisor $E=A \cap B$. It is the basechange via $M_{i j} \times_{T^{*} F l_{i j}} M_{i j} \rightarrow K_{i j} \times_{T^{*} F l_{i j}} K_{i j}$ of

$$
M_{i j} \times_{K_{j i}} M_{i j} \rightarrow K_{i j} \times_{T^{*} F l_{i j}} K_{i j},
$$

which by Lemma 4.9 is the blow-up of $K_{i j} \times_{T^{*} F l_{i j}} K_{i j}$ in the diagonal $K_{i j}$. Since this diagonal has codimension two, $\mathcal{O}(E)$ is the relative canonical bundle, which by computation is $\left(\omega_{F l_{i} / F l_{i j}}^{-1}, N_{j}, N_{i}, 0\right)$ (written in terms of the four factors of $B(57))$.

Therefore the line bundle $\left.\mathcal{O}\left(N_{i}, 0,0\right)(0, D)\right|_{B}=\mathcal{O}_{B}\left(N_{i}, N_{j}, N_{i}, 0\right)$ is isomorphic to $\left(\omega_{F l_{i} / F l_{i j}}\left(N_{i}\right), 0,0,0\right)(E)=\left(\omega_{F l / F l_{i j}}, 0,0,0\right)(E)$. Thus $(61)$ is

$$
\pi_{13 *}\left(\mathcal{O}_{B}\left(\omega_{F l / F l_{i j}}, 0,0,0\right)\right) \rightarrow \pi_{13 *}\left(\mathcal{O}_{B}\left(\omega_{F l / F l_{i j}}, 0,0,0\right)(E)\right),
$$

which is an isomorphism with both equal to $\mathcal{O}_{M_{i j} \times_{T^{*} F l_{i j}} M_{i j}}\left(\omega_{F l / F l_{i j}}, 0\right)$ since $E \subset B$ is exceptional.

So now (59) follows from (60) and (61), and (58) has become

$$
U_{i} \star U_{j} \star U_{i} \cong \mathcal{O}_{N_{i} \times_{K_{i}} N_{i}}\left(N_{i}, 0\right)[-3] \oplus \mathcal{O}_{M_{i j} \times_{T^{*} F l_{i j}} M_{i j}}\left(\omega_{F l / F l_{i j}}, 0\right)[-3] .
$$

But this is $U_{i}[-2] \oplus U_{i j}$, as claimed. 
Finally we describe the map id $\star \mathrm{ev}_{j} \star \mathrm{id}$. By (55) we consider (the shift by $[-2]$ of) the connecting homomorphism of the exact sequence

$$
\begin{aligned}
0 \rightarrow \mathcal{O}_{K \times N_{i} \times K_{i} N_{i}}\left(0, N_{i}, 0\right) & \rightarrow \mathcal{O}_{\left(K \times N_{i} \times K_{i} N_{i}\right) \cup_{K \times N_{i j} \times K_{i} N_{i}}\left(K \times N_{j} \times_{K_{j}} N_{i j} \times K_{i} N_{i}\right)}(0, D) \\
& \rightarrow \mathcal{O}_{K \times N_{j} \times K_{j} N_{i j} \times K_{i} N_{i}}(0, D) \rightarrow 0
\end{aligned}
$$

We must take the derived tensor product with $\mathcal{O}_{N_{i} \times_{K_{i}} N_{i}}\left(N_{i}, 0,0\right)[-1]$ and then apply $\pi_{13 *}$. This derived tensor product has Tor $_{0}$ and Tor $_{1}$ terms, as shown above; the $\operatorname{Tor}_{0}$ terms (i.e. the ordinary tensor product) sit inside the exact sequence

$$
\begin{aligned}
& 0 \rightarrow \mathcal{O}_{N_{i} \times K_{i}} N_{i} \times_{K_{i}} N_{i}\left(N_{i}, N_{i}, 0\right) \rightarrow \mathcal{O}_{\left(N_{i} \times K_{i} N_{i} \times_{K_{i}} N_{i}\right) \cup B}\left(N_{i}, 0,0\right)(0, D) \\
& \rightarrow \mathcal{O}_{A \cup B}\left(N_{i}, 0,0\right)(0, D) \rightarrow 0,
\end{aligned}
$$

in the above notation. Only the last term arises from a transverse intersection; i.e. the last term is the full derived tensor product.

Now by mapping the complexes representing the full tensor products to this exact sequence of $\operatorname{Tor}_{0} \mathrm{~s}$, a simple argument valid in any derived category shows the following. The connecting homomorphism of the above exact sequence is the same as the connecting homomorphism in the exact triangle of full tensor products, composed with the projection from the full tensor product on the left-hand side to its Tor $_{0}$ term. But the map $b$ of Proposition 4.5 is precisely $\left(\pi_{13 *}\right.$ of $)$ this projection from $\pi_{12}^{*} U_{i} \otimes \pi_{23}^{*} U_{i}$ to $\operatorname{Tor}_{0}\left(\pi_{12}^{*} U_{i}, \pi_{23}^{*} U_{i}\right)$.

So we take $\pi_{13 *}$ of the exact sequence (63). We showed above that the inclusion of $U_{i}[-2]$ into $U_{i} \star U_{j} \star U_{i} \cong U_{i}[-2] \oplus U_{i j}$ is induced by the inclusion of $\mathcal{O}_{A}\left(N_{i}, 0,0\right)(0, D)(-E) \cong \mathcal{O}_{A}\left(N_{i}, 0,0\right)$ (by $\left.(62)\right)$ into $\mathcal{O}_{A \cup B}\left(N_{i}, 0,0\right)(0, D)$, whose fiber product with (63) gives the exact sequence

$$
\begin{aligned}
& 0 \rightarrow \mathcal{O}_{N_{i} \times K_{i} N_{i} \times_{K_{i}} N_{i}}\left(N_{i}, N_{i}, 0\right) \rightarrow \mathcal{I}_{E \subset N_{i} \times K_{i} N_{i} \times_{K_{i}} N_{i}}\left(N_{i}, 0,0\right)(0, D) \\
& \rightarrow \mathcal{O}_{A}\left(N_{i}, 0,0\right) \rightarrow 0 .
\end{aligned}
$$

Taking $\pi_{13 *}$ of this sequence gives an isomorphism

$$
R^{0} \pi_{13 *} \mathcal{O}_{A}\left(N_{i}, 0,0\right) \cong R^{1} \pi_{13 *} \mathcal{O}_{N_{i} \times K_{i}} N_{i} \times_{K_{i}} N_{i}\left(N_{i}, N_{i}, 0\right)
$$

which by the above working is the morphism $\left.b \circ\left(\mathrm{id} \star \operatorname{ev}_{j} \star \mathrm{id}\right)\right|_{U_{i}[-2]}: U_{i}[-2] \stackrel{\sim}{\longrightarrow}$ $U_{i}[-2]$ after shifting by $[-2]$.

Remark. One can show that the previous result is equivalent to the following fact about Mukai flops. (We restrict attention to the $n=3$ case for sake of exposition; in higher dimensions we just get a family version of this case.) We let $N_{12}$ be the total space of the $\mathcal{O}(-1,-1)$-bundle over $F l\left(\mathbb{C}^{3}\right)$. This has maps $q_{1}, q_{2}$ to $T^{*}\left(\mathbb{P}^{2}\right)^{*}, T^{*} \mathbb{P}^{2}$ which are both the blow-up of their image in the zero section. Then on $D^{b}\left(T^{*} \mathbb{P}^{2}\right)$, $q_{2 *} \omega q_{1}^{*} q_{1 *} q_{2}^{*}=\mathrm{id} \oplus H^{*}\left(\iota_{\mathbb{P}^{2}}^{*}(\cdot)(-1)\right) \otimes \iota_{\mathbb{P}^{2} *} \mathcal{O}_{\mathbb{P}^{2}}(-2)$, where $\omega$ is the canonical bundle of $N_{12}$ (and therefore of the $q_{i}$ ). This gives a perhaps more conceptual reason why $q_{1 *} q_{2}^{*}: D^{b}\left(T^{*} \mathbb{P}^{2}\right) \rightarrow D^{b}\left(T^{*}\left(\mathbb{P}^{2}\right)^{*}\right)$ is not an equivalence $[\mathbf{K w}, \mathbf{N m}]$; if it were its adjoint $q_{2 *} \omega q_{1}^{*}$ would be its inverse. 
Proposition 4.11. The compositions

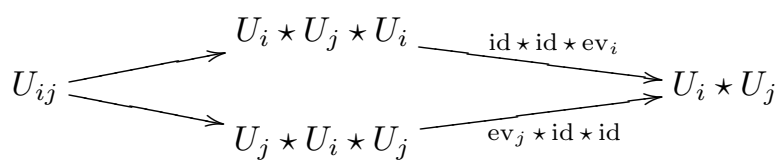

are the same map, which we call $\tau_{i j}$.

Proof. The key to this result is that there is a canonical morphism $\tau_{i j}$ in

$$
\operatorname{Hom}^{0}\left(U_{i j}, U_{i} \star U_{j}\right)=\operatorname{Ext}^{1}\left(\mathcal{O}_{M_{i j} \times_{T^{*} F l_{i j}} M_{i j}}\left(\omega_{F l / F l_{i j}}, 0\right), \mathcal{O}_{N_{j} \times_{K_{j}} N_{i j} \times_{K_{i}} N_{i}}(D)\right) .
$$

Letting $P$ and $Q$ denote $N_{j} \times_{K_{j}} N_{i j} \times_{K_{i}} N_{i}$ and $M_{i j} \times_{T^{*} F l_{i j}} M_{i j}$ respectively, it is the connecting homomorphism of the standard exact sequence

$$
0 \rightarrow \mathcal{O}_{P}(D) \rightarrow \mathcal{O}_{P \cup Q}\left(2 N_{i}+2 N_{j}, 0\right) \rightarrow \mathcal{O}_{Q}\left(\omega_{F l / F l_{i j}}, 0\right) \rightarrow 0 .
$$

Here we are using a number of easily checked facts. Firstly, on $P, \mathcal{O}(D)(54)$ is isomorphic to $\mathcal{O}\left(\omega_{F l / F l_{i j}},-N_{i}-N_{j}, 0\right)$ (written with respect to the three factors of $\left.N_{j} \times_{K_{j}} N_{i j} \times_{K_{i}} N_{i}\right)$. This can be proved by expanding everything in terms of the $L_{i}$ using (20) and $\omega_{F l / F l_{i j}} \cong L_{i}^{-2} L_{j}^{-2} L_{i-1} L_{j+1}=\mathcal{O}\left(2 N_{i}+2 N_{j}\right)$, and then moving $L_{k \neq j}$ across the first fiber product using the fact that such line bundles are pulled back from $K_{j}$.

Secondly, $P \cap Q=M_{i j} \times_{K_{i j}} M_{i j} \times_{K_{j i}} M_{i j}$ is a divisor in both $P$ and $Q$, and as a divisor in $Q$ it defines the line bundle $\mathcal{O}_{Q}\left(0, N_{i}+N_{j}, 0\right)$. This is because $P \cap Q \subset Q$ is the pullback of $M_{i j} \subset N_{i j}$ under $N_{j} \times_{K_{j}} N_{i j} \times_{K_{i}} N_{i} \rightarrow N_{i j}$, and $\mathcal{O}_{N_{i j}}\left(M_{i j}\right)$ is $\left.\mathcal{O}\left(N_{i}+N_{j}\right)\right|_{N_{i j}}(62)$.

By symmetry, both maps of Proposition 4.11 must be the same multiple of this canonical morphism $\tau_{i j}$, but for completeness we check that this multiple is 1 .

Using the description (31) of $U_{i} \rightarrow \mathcal{O}_{\Delta}$ as the connecting homomorphism of the exact sequence

$$
0 \rightarrow \mathcal{O}_{\Delta} \rightarrow \mathcal{O}_{\Delta \cup\left(N_{i} \times_{K_{i}} N_{i}\right)}\left(N_{i}, 0\right) \rightarrow \mathcal{O}_{N_{i} \times_{K_{i}} N_{i}}\left(N_{i}, 0\right) \rightarrow 0,
$$

we pull back by $\pi_{12}^{*}$ to $K \times K \times K$, tensor with $\pi_{23}^{*}\left(U_{i} \star U_{j}\right)$ and pushdown by $\pi_{13 *}$ to give id $\star \mathrm{id} \star \mathrm{ev}_{i}: U_{i} \star U_{j} \star U_{i} \rightarrow U_{i} \star U_{j}$. The proof of Proposition $4.10 \mathrm{com}-$ putes this tensor product and pushdown in detail so we follow our morphism (31) through that proof. We see that $\mathrm{id} \star \mathrm{id} \star \mathrm{ev}_{i}$ is represented by $\pi_{13 *}$ of the connecting homomorphism (shifted by $[-3]$ ) of the exact sequence

$$
\begin{aligned}
0 \rightarrow \mathcal{O}_{\Delta_{N_{j}} \times K_{j}} N_{i j} \times_{K_{i}} N_{i} & \left(0, N_{j}, N_{i}, 0\right) \\
& \rightarrow \mathcal{O}_{\left(\Delta_{N_{j}} \times_{K_{j}} N_{i j} \times_{K_{i}} N_{i}\right) \cup(A \cup B)}\left(N_{i}, N_{j}, N_{i}, 0\right) \rightarrow \mathcal{O}_{A \cup B}\left(N_{i}, N_{j}, N_{i}, 0\right) \rightarrow 0,
\end{aligned}
$$

in the notation of the proof of Proposition 4.10.

$U_{i j} \rightarrow U_{i} \star U_{j} \star U_{i}$ is induced by the inclusion of $\mathcal{O}_{B}\left(N_{i}, N_{j}, N_{i}, 0\right)(-E)$ into the last sheaf in the above exact sequence. Therefore we pull back the exact sequence 
by this map to give

$$
\begin{aligned}
0 & \rightarrow \mathcal{O}_{\Delta_{N_{j}} \times_{K_{j}} N_{i j} \times_{K_{i}} N_{i}}\left(0, N_{j}, N_{i}, 0\right) \\
& \rightarrow \mathcal{O}_{\left(\Delta_{N_{j}} \times_{K_{j}} N_{i j} \times_{K_{i}} N_{i}\right) \cup B}\left(N_{i}, N_{j}, N_{i}, 0\right)(L) \rightarrow \mathcal{O}_{B}\left(N_{i}, N_{j}, N_{i}, 0\right)(L) \rightarrow 0,
\end{aligned}
$$

whose connecting homomorphism gives (after applying $\pi_{13 *}[-3]$ ) the composition $U_{i j} \rightarrow U_{i} \star U_{j} \star U_{i} \rightarrow U_{i} \star U_{j}$ we require. Here

$$
L=\mathcal{O}_{\left(\Delta_{N_{j}} \times_{K_{j}} N_{i j} \times_{K_{i}} N_{i}\right) \cup B}\left(0, L_{j}^{-1} L_{j+1}, L_{j}^{-1} L_{i}, 0\right)
$$

is the line bundle defined by minus the divisor given by the intersection of $A$ with $\Delta_{N_{j}} \times_{K_{j}} N_{i j} \times_{K_{i}} N_{i}$.

Pushing down as in the proof of Proposition 4.10 gives the exact sequence (65), so the composition we require is its connecting homomorphism - our canonical morphism $\tau_{i j}$.

By symmetry the same is true of the other morphism.

Theorem 4.12. There is an isomorphism $U_{i} \star T_{j} \star T_{i} \cong T_{j} \star T_{i} \star U_{j}$ which intertwines the evaluation maps $\mathrm{ev}_{i} \star \mathrm{id} \star \mathrm{id}$ and $\mathrm{id} \star \mathrm{id} \star \mathrm{ev}_{j}$ from the two kernels to $T_{j} \star T_{i}$

Taking cones on these two maps, $T_{i} \star T_{j} \star T_{i} \cong T_{i} \star T_{j} \star T_{i}$ for $|i-j|=1$.

Proof. Expanding out $U_{i} \star T_{j} \star T_{i}$ gives the cone on

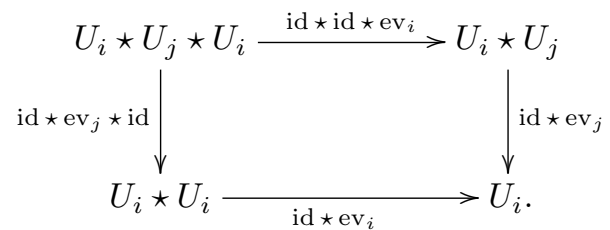

If we use $\mathrm{id} \star \mathrm{ev}_{i}$ to split $U_{i} \star U_{i}$ as in Proposition 4.5, then by Propositions 4.10, 4.11 and 4.5 we get

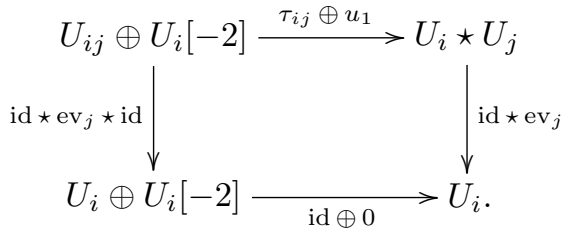

where the exact form of $u_{1}$ will not concern us.

We map

$$
\text { Cone }\left(U_{i j} \stackrel{\tau_{i j}}{\longrightarrow} U_{i} \star U_{j}\right)
$$

into the cone on (67) as follows. $U_{i j}$ maps to the first term of (67) as the first summand. $U_{i} \star U_{j}$ maps to the top right term via the identity and to the bottom left term by id $\star \mathrm{ev}_{j} \oplus 0$. To check this is really a morphism in $D^{b}(K \times K)$ we need only check that the first vertical arrow of (67) acts on $U_{i j}$ as $\left(\mathrm{id} \star \mathrm{ev}_{j}\right) \circ \tau_{i j} \oplus 0$. But 
the first factor of this map is determined by the commutativity of (66), while for the second we note that

$$
\operatorname{Hom}^{0}\left(U_{i j}, U_{i}[-2]\right) \cong \operatorname{Hom}^{0}\left(\mathcal{O}_{M_{i j} \times_{T^{*} F l_{i j}} M_{i j}}\left(\omega_{F l / F l_{i j}}, 0\right), \mathcal{O}_{N_{i} \times_{K_{i}} N_{i}}\left(N_{i}, 0\right)\right)
$$

vanishes, since there are no morphisms $\mathcal{O}_{A} \rightarrow \mathcal{O}_{B}$ unless there is an irreducible component of $B$ contained in $A$.

This morphism is a quasi-isomorphism, since by the second part of Proposition 4.10 what remains is

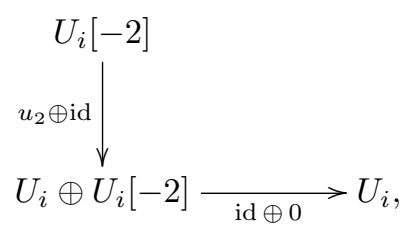

for some $u_{2}$, by Proposition 4.10. But this is quasi-isomorphic to zero.

So by symmetry there is a similar quasi-isomorphism from Cone $\left(U_{i j} \stackrel{\tau_{i j}}{\longrightarrow} U_{i} \star U_{j}\right)$ to $T_{j} \star T_{i} \star U_{j}$, making the latter isomorphic to $U_{i} \star T_{j} \star T_{i}$.

Composing $\mathrm{ev}_{i} \star \mathrm{id} \star \mathrm{id}: U_{i} \star T_{j} \star T_{i} \rightarrow T_{j} \star T_{i}$ with the quasi-isomorphism from (68) gives the morphism of cones

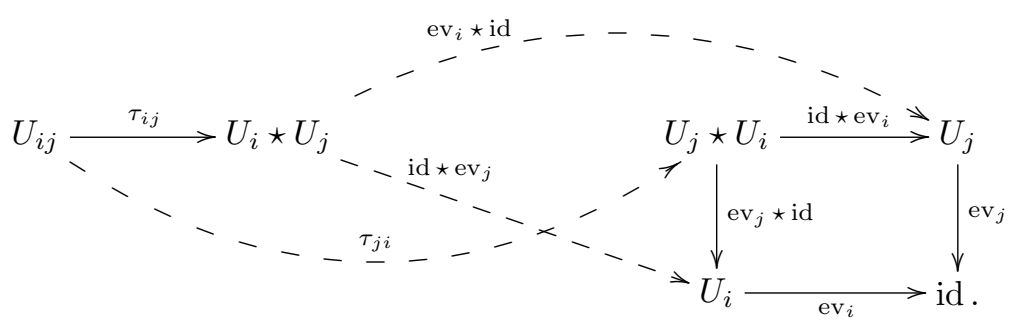

But by similar working this is exactly the morphism of cones that comes from composing $\mathrm{id} \star \mathrm{id} \star \mathrm{ev}_{j}: T_{j} \star T_{i} \star U_{j} \rightarrow T_{j} \star T_{i}$ with the quasi-isomorphism from

$$
\text { Cone }\left(U_{i j} \stackrel{\tau_{i j}}{\longrightarrow} U_{i} \star U_{j}\right)
$$

to $T_{j} \star T_{i} \star U_{j}$.

Remark. We note in passing that the faithfulness results of $[\mathbf{K S}, \mathbf{S T}]$ can be used to show that our braid group action on $D^{b}\left(T^{*} F l\right)$ is faithful. The transverse slice to a subregular nilpotent matrix gives a surface $X$ in $K=T^{*} F l$ with an $A_{n}$ chain of -2-spheres $C_{i}$. The $C_{i}$ are the transverse intersection of the subvarieties $N_{i}$ with the surface. It follows that the Fourier-Mukai kernels $(29,32)$ for $U_{i}$ and $T_{i}$ restrict, on $X \times X \subset K \times K$, to the standard kernels for the Dehn twists [ST] about the structure sheaves $\mathcal{O}_{C_{i}}$ of the $C_{i}$-see $(77,78)$ where these are reviewed.

Therefore the two types of derived category twist, and the braid group actions they generate, are intertwined by restriction from $K$ to $X$; i.e. the following diagram 
commutes.

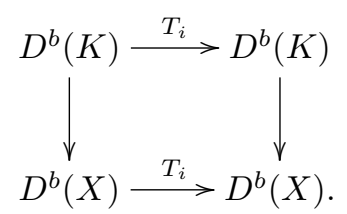

Thus the faithfulness of the lower action implies the same of the upper action.

\subsection{Extension to the affine braid group}

As noted in [ST, Example 3.9], the braid group action of that paper on the derived category of coherent sheaves on a surface with an $A_{n-1}$-chain of -2-curves $C_{i}$ can be extended to an action of the affine braid group. If we take generators of the braid group to be $T_{i} \stackrel{\text { def }}{=} T_{\mathcal{O}_{C_{i}}}$, the Dehn twists about the structure sheaves $\mathcal{O}_{C_{i}}$ described in Section 5.1, then we define the extra generator

$$
T=T_{0}=T_{n} \stackrel{\text { def }}{=} T_{\mathcal{O}_{E}(1, \ldots, 1)} .
$$

This is the Dehn twist about $\mathcal{O}_{E}(1, \ldots, 1)$, the structure sheaf of the entire chain $E=\cup_{i} C_{i}$ twisted by the line bundle on $E$ which has degree 1 on each $C_{i}$. This commutes with $T_{i}$ for $2 \leqslant i \leqslant n-2$ and braids with $T_{1}$ and $T_{n-1}$, so defines an affine braid group action.

Now it is an easy calculation that $T_{\mathcal{O}_{C_{i}}}\left(\mathcal{O}_{C_{i+1} \cup \ldots \cup C_{n-1}}(0,1, \ldots, 1)\right)$ is isomorphic to $\mathcal{O}_{C_{i} \cup \ldots \cup C_{n-1}}(0,1, \ldots, 1)$. Thus, by induction,

$$
\mathcal{L}_{1} T_{1} T_{2} \ldots T_{n-2}\left(\mathcal{O}_{C_{n-1}}\right) \cong \mathcal{O}_{E}(1,1 \ldots, 1)
$$

where $\mathcal{L}_{1}$ is the functor of tensoring with a line bundle $L_{1}$ whose restriction to $E$ is $\mathcal{O}_{E}(1,0, \ldots, 0)$ (for the purposes of this motivational section we may assume that $\mathcal{O}_{E}(1,0, \ldots, 0)$ extends to a line bundle $L_{1}$ on the surface).

Thus, by [ST, Lemma 2.11], the twist $T=T_{\mathcal{O}_{E}(1, \ldots, 1)}$ can be written as

$$
T=\operatorname{ad}\left(\mathcal{L}_{1} T_{1} \ldots T_{n-2}\right) T_{\mathcal{O}_{C_{n-1}}}=\left(\mathcal{L}_{1} T_{1} \ldots T_{n-2}\right) T_{n-1}\left(T_{n-2}^{\prime} \ldots T_{1}^{\prime} \mathcal{L}_{1}^{-1}\right) .
$$

Returning to the cotangent bundle of the flag variety, the definition (69) does not easily generalise to our situation, but (70) certainly does.

Define the kernel $T=T_{0}=T_{n} \in D^{b}(K \times K)$ to be

$$
\left(\left(T_{1} \star T_{2} \star \ldots \star T_{n-2}\right) \star T_{n-1} \star\left(T_{n-2}^{\prime} \star \ldots \star T_{2}^{\prime} \star T_{1}^{\prime}\right)\right)\left(L_{1}^{-1}, L_{1}\right) .
$$

From now on we will often suppress the $\star s$ in similar long formulae. We also introduce the notation that if $L$ is a line bundle on $K$ then $\mathcal{L} \stackrel{\text { def }}{=} \mathcal{O}_{\Delta} \otimes \pi_{1}^{*} L=$ $\mathcal{O}_{\Delta} \otimes \pi_{2}^{*} L$ is the kernel for $L \otimes(\cdot)$. That is, $\mathcal{L}$ is the line bundle $L$ supported on the diagonal of $K \times K$, and we have $S \star \mathcal{L}=S(L, 0), \mathcal{L} \star S=S(0, L)$ for any kernel $S$. Thus (71) can be rewritten

$$
T=\left(\mathcal{L}_{1} T_{1} T_{2} \ldots T_{n-2}\right) T_{n-1}\left(T_{n-2}^{\prime} \ldots T_{2}^{\prime} T_{1}^{\prime} \mathcal{L}_{1}^{-1}\right) .
$$

For $i \neq j, L_{i}$ is pulled back from $K_{j}$, so the line bundles $\left(L_{i}, 0\right)$ and $\left(0, L_{i}\right)$ are isomorphic on restriction to $\Delta \cup\left(N_{j} \times_{K_{j}} N_{j}\right)$. Thus $T_{j}\left(0, L_{i}\right)=T_{j}\left(L_{i}, 0\right)$, i.e. 
$\mathcal{L}_{i} \star T_{j}=T_{j} \star \mathcal{L}_{i}$ for $i \neq j$. Using this and the braiding we have already proved, we automatically get the following.

Proposition 4.13. For $2 \leqslant i \leqslant n-2$, we have $T \star T_{i} \cong T_{i} \star T$.

Proof. As explained above we can commute $T_{i}$ past $\mathcal{L}_{1}^{-1}$ in (72), and then past $T_{j}^{\prime}, j<i-1$ using Propositions 4.7 and 4.6. This gives

$$
T \star T_{i} \cong\left(\mathcal{L}_{1} T_{1} T_{2} \ldots T_{n-2}\right) T_{n-1}\left(T_{n-2}^{\prime} \ldots T_{i}^{\prime} T_{i-1}^{\prime}\right) T_{i}\left(T_{i-2}^{\prime} \ldots T_{2}^{\prime} T_{1}^{\prime} \mathcal{L}_{1}^{-1}\right) .
$$

But $T_{i}$ and $T_{i-1}$ braid, so that $T_{i}^{\prime} T_{i-1}^{\prime} T_{i} \cong T_{i-1} T_{i}^{\prime} T_{i-1}^{\prime}$, and then this new $T_{i-1}$ commutes past $T_{j}^{\prime}$ and $T_{j}$ for $j>i$, yielding

$$
\left(\mathcal{L}_{1} T_{1} \ldots T_{i-1} T_{i}\right) T_{i-1}\left(T_{i+1} \ldots T_{n-2}\right) T_{n-1}\left(T_{n-2}^{\prime} \ldots T_{i}^{\prime} T_{i-1}^{\prime} T_{i-2}^{\prime} \ldots T_{1}^{\prime} \mathcal{L}_{1}^{-1}\right) .
$$

So using $T_{i-1} T_{i} T_{i-1} \cong T_{i} T_{i-1} T_{i}$ we get

$$
\left(\mathcal{L}_{1} T_{1} \ldots T_{i-2} T_{i} T_{i-1} T_{i} T_{i+1} \ldots T_{n-2}\right) T_{n-1}\left(T_{n-2}^{\prime} \ldots T_{1}^{\prime} \mathcal{L}_{1}^{-1}\right) .
$$

Commuting $T_{i}$ past $\mathcal{L}_{1} T_{1} \ldots T_{i-2}$ gives $T_{i} \star T$, as required.

Similarly the next braid relation is also a pure algebraic formality now that we know that $T_{n-1}$ commutes with $\mathcal{L}_{1}$ :

Proposition 4.14. $T \star T_{n-1} \star T \cong T_{n-1} \star T \star T_{n-1}$.

Proof. $T \star T_{n-1} \star T$ is

$$
\left(\mathcal{L}_{1} T_{1} \ldots T_{n-2}\right) T_{n-1}\left(T_{n-2}^{\prime} \ldots T_{1}^{\prime} \mathcal{L}_{1}^{-1}\right) T_{n-1}\left(\mathcal{L}_{1} T_{1} \ldots T_{n-2}\right) T_{n-1}\left(T_{n-2}^{\prime} \ldots T_{1}^{\prime} \mathcal{L}_{1}^{-1}\right) .
$$

We commute the middle $T_{n-1}$ past $T_{n-3}^{\prime} \ldots T_{1}^{\prime} \mathcal{L}_{1}^{-1}$ to give

$$
\left(\mathcal{L}_{1} T_{1} \ldots T_{n-2}\right) T_{n-1} T_{n-2}^{\prime} T_{n-1} T_{n-2} T_{n-1}\left(T_{n-2}^{\prime} \ldots T_{1}^{\prime} \mathcal{L}_{1}^{-1}\right) .
$$

Because $T_{n-2}$ and $T_{n-1}$ braid, $T_{n-2}^{\prime} T_{n-1} T_{n-2} T_{n-1} T_{n-2}^{\prime} \cong T_{n-1}$, leaving

$$
\left(\mathcal{L}_{1} T_{1} \ldots T_{n-2}\right) T_{n-1} T_{n-1}\left(T_{n-3}^{\prime} \ldots T_{1}^{\prime} \mathcal{L}_{1}^{-1}\right) .
$$

Similarly, $T_{n-1} \star T \star T_{n-1}$ is

$$
\left(\mathcal{L}_{1} T_{1} \ldots T_{n-3}\right) T_{n-1} T_{n-2} T_{n-1} T_{n-2}^{\prime} T_{n-1}\left(T_{n-3}^{\prime} \ldots T_{1}^{\prime} \mathcal{L}_{1}^{-1}\right),
$$

which by the braiding of $T_{n-2}$ and $T_{n-1}$ is

$$
\left(\mathcal{L}_{1} T_{1} \ldots T_{n-3}\right) T_{n-2} T_{n-1} T_{n-1}\left(T_{n-3}^{\prime} \ldots T_{1}^{\prime} \mathcal{L}_{1}^{-1}\right) .
$$

But this is (73).

The final braid relation is not quite such a formality, but also requires the observation from $(32,40)$ that $T_{i}^{\prime} \cong T_{i}\left(L_{i} L_{i-1}^{-1}, L_{i} L_{i+1}^{-1}\right)$, i.e.

$$
T_{i}^{\prime} \cong \mathcal{L}_{i} \star \mathcal{L}_{i+1}^{-1} \star T_{i} \star \mathcal{L}_{i} \star \mathcal{L}_{i-1}^{-1} .
$$

Proposition 4.15. $T \star T_{1} \star T \cong T_{1} \star T \star T_{1}$. 
Proof. By the same reasoning as in Proposition 4.14, $T_{1}$ braids with

$$
S \stackrel{\text { def }}{=}\left(\mathcal{L}_{n-1} T_{n-1} \ldots T_{2}\right) T_{1}\left(T_{2}^{\prime} \ldots T_{n-1}^{\prime} \mathcal{L}_{n-1}^{-1}\right)
$$

i.e. $T_{1} \star S \star T_{1} \cong S \star T_{1} \star S$. But we know that $T_{1}$ commutes with $\mathcal{L}_{2}$, so the above also equals

$$
S=\left(\mathcal{L}_{n-1} T_{n-1} \ldots T_{2} \mathcal{L}_{2}\right) T_{1}\left(\mathcal{L}_{2}^{-1} T_{2}^{\prime} \ldots T_{n-1}^{\prime} \mathcal{L}_{n-1}^{-1}\right)
$$

We can rewrite $T(71)$ as

$$
T=\left(\mathcal{L}_{1} T_{n-1}^{\prime} \ldots T_{2}^{\prime}\right) T_{1}\left(T_{2} \ldots T_{n-1} \mathcal{L}_{1}^{-1}\right),
$$

by using the braid relations first for $T_{n-1}$ and $T_{n-2}$, then $T_{n-2}$ and $T_{n-3}$ on the result, all the way down to $T_{2}$ and $T_{1}$. Commuting $\mathcal{L}_{1}$ with $T_{i}$ for all $i \geqslant 2$, this can be rewritten

$$
T=\left(T_{n-1}^{\prime} \ldots T_{2}^{\prime} \mathcal{L}_{1}\right) T_{1}\left(\mathcal{L}_{1}^{-1} T_{2} \ldots T_{n-1}\right) .
$$

But by (74), $T_{n-1}^{\prime} \ldots T_{2}^{\prime} \mathcal{L}_{1}=\mathcal{L}_{n-1} \mathcal{L}_{n}^{-1} T_{n-1} T_{n-2} \ldots T_{2} \mathcal{L}_{2}$ and $L_{n}$ is trivial, so we can rewrite (76) as (75). Thus $T=S$, which braids with $T_{1}$.

\section{Actions of the braid cobordism category on derived cate- gories of coherent sheaves}

We now extend the braid group actions of $[\mathbf{S T}]$ (on surfaces $X$ with $A_{n}$-chains of -2-spheres $C_{i}$ ) and Section 4 (on $T^{*} F l$ ) to actions of the braid cobordism category. The latter case proceeds along similar lines to the former, which we tackle now.

We are guided by the action of the braid cobordism category on the homotopy category $\mathcal{C}\left(A_{n}\right)$ of Section 2 . Using the formality result of $[\mathbf{K S}]$ it is shown in $[\mathbf{S T}]$ that the differential graded algebra $\operatorname{RHom}\left(\oplus_{i} \mathcal{O}_{C_{i}}, \oplus_{i} \mathcal{O}_{C_{i}}\right)$ is quasi-isomorphic to $A_{n} \otimes \mathbb{C}$, so that its derived category of differential graded modules is equivalent is that of $A_{n} \otimes \mathbb{C}$. Under this equivalence, $\mathcal{O}_{C_{i}}$ is mapped to $P_{i}$, the twists $T_{i}, T_{i}^{\prime}$ reviewed below $(77,78)$ map to $R_{i}, R_{i}^{\prime}$, and $\mathrm{ev}_{i}, \mathrm{ev}_{i}^{\prime}$ become $\beta_{i}, \gamma_{i}$.

So the work in the next section is to lift the positive $(10,11)$ and negative $(12$, 13) braid moves on $\mathcal{C}\left(A_{n}\right)$ to $D^{b}(X)$. This is done in (105) and (106) respectively. The positive moves are fairly easy, the negative moves require some technical work, in particular Proposition 5.5.

\subsection{Surfaces with chains of rational curves}

Let $X$ be a smooth quasi-projective surface with an $A_{n}$-chain of -2 -spheres $C_{i}$ : the exceptional set of the minimal resolution of an $A_{n}$ surface singularity. Then $\omega_{X}$ is trivial in a neighbourhood of $E=\cup_{i} C_{i}$; for simplicity we will assume that $\omega_{X}$ is globally trivial and $i x$ a trivialisation $\theta \in H^{0}\left(\omega_{X}\right)$ (with a little more work what follows can be pushed through using a trivialisation defined only on some Zariski open subset $U \supset E$ of $X$ ).

A (weak) braid group action is constructed on the bounded derived category of coherent sheaves $D^{b}(X)$ on $X$ in $[\mathbf{S T}]$, with generators $T_{i}$ fitting into distinguished 
triangles

$$
U_{i}(\mathcal{F}) \stackrel{\text { def }}{=} \mathcal{O}_{C_{i}} \otimes \operatorname{RHom}\left(\mathcal{O}_{C_{i}}, \mathcal{F}\right) \stackrel{\text { ev }_{i}}{\longrightarrow} \mathcal{F} \rightarrow T_{i}(\mathcal{F}),
$$

and inverses $T_{i}^{\prime}$

$$
T_{i}^{\prime}(\mathcal{F}) \rightarrow \mathcal{F} \stackrel{\mathrm{ev}_{i}^{\prime}}{\longrightarrow} \mathcal{O}_{C_{i}} \otimes \operatorname{RHom}\left(\mathcal{O}_{C_{i}}, \mathcal{F}\right)[2]=U_{i}(\mathcal{F})[2]
$$

Using very similar (but easier) methods to those in Section 4.2 one shows that these are represented by the Fourier-Mukai kernels $U_{i} \stackrel{\text { def }}{=} \mathcal{O}_{C_{i}}^{\vee} \otimes \mathcal{O}_{C_{i}} \cong \mathcal{O}_{C_{i} \times C_{i}}\left(C_{i}, 0\right)[-1]$,

$$
T_{i} \stackrel{\text { def }}{=} \mathcal{O}_{\Delta \cup\left(C_{i} \times C_{i}\right)}\left(C_{i}, 0\right) \text { and } T_{i}^{\prime} \stackrel{\text { def }}{=} \mathcal{O}_{\Delta \cup\left(C_{i} \times C_{i}\right)}\left(L_{i}\right)
$$

on $X \times X$ (cf. (32) and (40)). Here $L_{i}$ is $\mathcal{O}_{\Delta}$ glued across $\Delta_{C_{i}}$ to

$$
\mathcal{O}_{C_{i} \times C_{i}}\left(C_{i}, 0\right)\left(-\Delta_{C_{i}}\right),
$$

using the fact that the latter restricts on $\Delta_{C_{i}}$ to $\Omega_{C_{i}}^{1}\left(C_{i}\right)$ which is isomorphic to $\mathcal{O}_{\Delta_{C_{i}}}$ using adjunction and the trivialisation $\theta$ of $\left.\omega_{X}\right|_{C_{i}}$.

The exact triangles (77) and (78) then arise from the exact sequences of FourierMukai kernels

$$
0 \rightarrow \mathcal{O}_{\Delta} \rightarrow \mathcal{O}_{\Delta \cup\left(C_{i} \times C_{i}\right)}\left(C_{i}, 0\right) \stackrel{r}{\rightarrow} \mathcal{O}_{C_{i} \times C_{i}}\left(C_{i}, 0\right) \rightarrow 0
$$

and

$$
0 \rightarrow \mathcal{O}_{C_{i} \times C_{i}}\left(C_{i}, 0\right) \stackrel{i}{\rightarrow} \mathcal{O}_{\Delta \cup\left(C_{i} \times C_{i}\right)}\left(L_{i}\right) \rightarrow \mathcal{O}_{\Delta} \rightarrow 0 .
$$

Taking the Yoneda product of these two extensions gives the connecting morphism $\mathrm{ev}_{i} \circ \mathrm{ev}_{i}^{\prime}: \mathcal{O}_{\Delta} \rightarrow \mathcal{O}_{\Delta}[2]$ in the exact triangle

$$
\mathcal{O}_{\Delta}[1] \rightarrow \text { Cone }\left(\mathcal{O}_{\Delta \cup\left(C_{i} \times C_{i}\right)}\left(C_{i}, 0\right) \stackrel{i \circ r}{\longrightarrow} \mathcal{O}_{\Delta \cup\left(C_{i} \times C_{i}\right)}\left(L_{i}\right)\right) \rightarrow \mathcal{O}_{\Delta}
$$

which arises from the exact sequence given by splicing (79) and (80) together:

$$
0 \rightarrow \mathcal{O}_{\Delta} \rightarrow \mathcal{O}_{\Delta \cup\left(C_{i} \times C_{i}\right)}\left(C_{i}, 0\right) \stackrel{i \circ r}{\longrightarrow} \mathcal{O}_{\Delta \cup\left(C_{i} \times C_{i}\right)}\left(L_{i}\right) \rightarrow \mathcal{O}_{\Delta} \rightarrow 0 .
$$

The sheaf $\mathcal{O}_{C_{i} \times C_{i}}\left(C_{i}, 0\right)\left(-\Delta_{C_{i}}\right)$ naturally injects into both terms of the Cone in (81); take cokernels to give the following.

Lemma 5.1. The natural transformation $\mathrm{ev}_{i} \circ \mathrm{ev}_{i}^{\prime}: \mathrm{id} \rightarrow \mathrm{id}[2]$ is represented by the morphism $\mathrm{ev}_{i} \circ \mathrm{ev}_{i}^{\prime}: \mathcal{O}_{\Delta} \rightarrow \mathcal{O}_{\Delta}[2]$ in the exact triangle

$$
\mathcal{O}_{\Delta}[1] \rightarrow \text { Cone }\left(\mathcal{O}_{\Delta}\left(\Delta_{C_{i}}\right) \stackrel{i \circ r}{\longrightarrow} \mathcal{O}_{\Delta \cup 2 \Delta_{C_{i}}}\right) \rightarrow \mathcal{O}_{\Delta} \rightarrow \mathcal{O}_{\Delta}[2]
$$

where $2 \Delta_{C_{i}}$ denotes the thickening of $\Delta_{C_{i}}$ in $C_{i} \times C_{i}$ only; i.e. the pushforward to $X \times X$ of the subscheme of $C_{i} \times C_{i}$ defined by the ideal sheaf $\mathcal{I}_{\Delta_{C_{i}}}^{2}$.

It is a result of Gerstenhaber and Schack $[\mathbf{K}]$ that there is a canonical isomorphism

$$
\operatorname{Ext}^{i+j}\left(\mathcal{O}_{\Delta}, \mathcal{O}_{\Delta}\right) \cong \bigoplus_{i+j=k} H^{i}\left(\mathcal{E} x t^{j}\left(\mathcal{O}_{\Delta}, \mathcal{O}_{\Delta}\right)\right) \cong \bigoplus_{i+j=k} H^{i}\left(\Lambda^{j} T_{\Delta}\right)
$$

In our situation, fixing $i+j=2$ and contracting with the trivialisation $\theta$ of $\omega_{X}$ 
gives

$$
\operatorname{Ext}^{2}\left(\mathcal{O}_{\Delta}, \mathcal{O}_{\Delta}\right) \cong H^{0}\left(\Omega_{X}^{2}\right) \oplus H^{1}\left(\Omega_{X}^{1}\right) \oplus H^{2}\left(\mathcal{O}_{X}\right) .
$$

Later it will be important that here we have fixed an identification of $T_{X}$ with the normal bundle to $\Delta$; our convention is that in the splitting $\left.T_{X \times X}\right|_{\Delta} \cong T_{X} \oplus T_{X}$ induced by the two factors, $T_{X}$ maps to the normal bundle as the image of

$$
T X \ni v \mapsto(v,-v) \in T_{X} \oplus T_{X} .
$$

As a divisor, $C_{i} \subset X$ defines a de Rham class $\left[C_{i}\right] \in H^{1}\left(\Omega_{X}^{1}\right)$ in the following way (which gives the usual cohomology class $\left[C_{i}\right] \in H^{1,1}(X)$ if $X$ is compact). Think of $H^{1}\left(\Omega_{X}^{1}\right)$ as $\operatorname{Ext}^{1}\left(\mathcal{O}_{X}, \Omega_{X}^{1}\right)$, which has a map from $\operatorname{Ext}^{1}\left(\mathcal{O}_{C_{i}}, \Omega_{X}^{1}\right)$ $=H^{0}\left(\mathcal{E} x t^{1}\left(\mathcal{O}_{C_{i}}, \Omega_{X}^{1}\right)\right)=H^{0}\left(\Omega_{X}^{1}\left(C_{i}\right)\right)=\operatorname{Hom}\left(T_{X}, \nu_{C_{i}}\right)$. The canonical element of the latter (projection of a tangent vector to the normal bundle) defines the extension

$$
0 \rightarrow \Omega_{X}^{1} \rightarrow \Omega_{X}^{1}\left(\log C_{i}\right) \rightarrow \mathcal{O}_{C_{i}} \rightarrow 0,
$$

where the last map takes the residue of logarithmic forms on $C_{i}$. The map $\mathcal{O}_{X} \rightarrow \mathcal{O}_{C_{i}}$ pulls this back to the extension

$$
0 \rightarrow \Omega_{X}^{1} \rightarrow \Omega_{X}^{1}\left(\log C_{i}\right) \times \mathcal{O}_{C_{i}} \mathcal{O}_{X} \rightarrow \mathcal{O}_{X} \rightarrow 0,
$$

whose extension class is $\left[C_{i}\right] \in H^{1}\left(\Omega_{X}^{1}\right)$.

Proposition 5.2. The class of $\mathrm{ev}_{i} \circ \mathrm{ev}_{i}^{\prime} \in \operatorname{Ext}^{2}\left(\mathcal{O}_{\Delta}, \mathcal{O}_{\Delta}\right)$ of Lemma 5.1 can be described, via the isomorphism (82), as the de Rham class

$$
\left[C_{i}\right] \in H^{1}\left(\Omega_{X}^{1}\right) \subset \operatorname{Ext}^{2}\left(\mathcal{O}_{\Delta}, \mathcal{O}_{\Delta}\right) .
$$

Proof. Dualising (84) shows that $\left[C_{i}\right] \in H^{1}\left(\Omega_{X}^{1}\right)=\operatorname{Ext}^{1}\left(T_{X}, \mathcal{O}_{X}\right)$ defines the extension

$$
0 \rightarrow \mathcal{O}_{X} \rightarrow \mathcal{F} \rightarrow T_{X} \rightarrow 0
$$

where $\mathcal{F}$ is the dual of

$$
\left\{(\sigma, f) \in \Omega_{X}^{1}\left(\log C_{i}\right) \oplus \mathcal{O}_{X}: \operatorname{Res}_{C_{i}} \sigma=\left.f\right|_{C_{i}} \in \mathcal{O}_{C_{i}}\right\}=\Omega_{X}^{1}\left(\log C_{i}\right) \times_{\mathcal{O}_{C_{i}}} \mathcal{O}_{X} .
$$

Thus it is easy to see that $\mathcal{F}$ is

$$
\left\{(v, s) \in T_{X} \oplus \mathcal{O}\left(C_{i}\right): \pi(v)=\left.s\right|_{C_{i}}\right\}=T_{X} \times_{\nu_{C_{i}}} \mathcal{O}\left(C_{i}\right),
$$

where $\pi: T_{X} \rightarrow \nu_{C_{i}}$ is the canonical projection to $\mathcal{O}_{C_{i}}\left(C_{i}\right)$. It therefore also sits inside the exact sequence

$$
0 \rightarrow T_{X}\left(-\log C_{i}\right) \rightarrow \mathcal{F} \rightarrow \mathcal{O}\left(C_{i}\right) \rightarrow 0,
$$

where $T_{X}\left(-\log C_{i}\right) \stackrel{\text { def }}{=} \operatorname{ker}\left(T_{X} \stackrel{\pi}{\rightarrow} \nu_{C_{i}}\right)$ is the dual of $\Omega_{X}^{1}\left(\log C_{i}\right)$.

Meanwhile $\mathcal{E} x t_{X \times X}^{1}\left(\mathcal{O}_{\Delta}, \mathcal{O}_{\Delta}\right) \cong \Omega_{\Delta}^{1}$ is represented by the universal extension

$$
0 \rightarrow \Omega_{\Delta}^{1} \rightarrow \mathcal{O}_{2 \Delta} \rightarrow \mathcal{O}_{\Delta} \rightarrow 0,
$$

which contraction with $\theta \in H^{0}\left(\omega_{X}\right)$ makes into

$$
0 \rightarrow T_{\Delta} \rightarrow \mathcal{O}_{2 \Delta} \rightarrow \mathcal{O}_{\Delta} \rightarrow 0 .
$$

Therefore $\left[C_{i}\right] \in H^{1}\left(\mathcal{E} x t^{1}\left(\mathcal{O}_{\Delta}, \mathcal{O}_{\Delta}\right)\right) \subset \operatorname{Ext}^{2}\left(\mathcal{O}_{\Delta}, \mathcal{O}_{\Delta}\right)$ is represented by the splicing 
together of the exact sequences (89) and (the pushforward to $\Delta$ of) (85):

$$
0 \rightarrow \mathcal{O}_{\Delta} \rightarrow \mathcal{F} \rightarrow \mathcal{O}_{2 \Delta} \rightarrow \mathcal{O}_{\Delta} \rightarrow 0 .
$$

Now $T_{X}\left(-\log C_{i}\right)$ injects into $\mathcal{F}(87)$ and under $\theta$ it injects into $\Omega_{X}^{1}$ as $\Omega_{X}^{1}\left(\log C_{i}\right)\left(-C_{i}\right)$, with quotient $\Omega_{C_{i}}^{1}$. Therefore it injects into $\mathcal{O}_{2 \Delta}$ with quotient $\mathcal{O}_{\Delta \cup 2 \Delta_{C_{i}}}$, with $C_{i}$ thickened only inside $C_{i} \times C_{i}$ as described in Lemma 5.1. So taking the quotient of the central two terms of (90) by $T_{X}\left(-\log C_{i}\right)$ gives, by (87), the exact sequence

$$
0 \rightarrow \mathcal{O}_{\Delta} \rightarrow \mathcal{O}_{\Delta}\left(\Delta_{C_{i}}\right) \rightarrow \mathcal{O}_{\Delta \cup 2 \Delta_{C_{i}}} \rightarrow \mathcal{O}_{\Delta} \rightarrow 0 .
$$

But this is exactly what represents $\mathrm{ev}_{i} \circ \mathrm{ev}_{i}^{\prime}$, by Lemma 5.1 .

So an element of $H^{1}\left(\Omega_{X}^{1}\right) \subset \operatorname{Ext}^{2}\left(\mathcal{O}_{\Delta}, \mathcal{O}_{\Delta}\right)=\operatorname{Hom}^{0}\left(\mathcal{O}_{\Delta}, \mathcal{O}_{\Delta}[2]\right)$ induces, by the Fourier-Mukai transform, a morphism $\mathcal{F} \rightarrow \mathcal{F}[2]$ for any $\mathcal{F} \in D^{b}(X)$. In particular, for every proper curve $D \subset X$ we induce an element of $\operatorname{Ext}^{2}\left(\mathcal{O}_{D}, \mathcal{O}_{D}\right)=H^{1}\left(\Omega_{D}^{1}\right)$, which is described as one might expect.

Lemma 5.3. The diagram

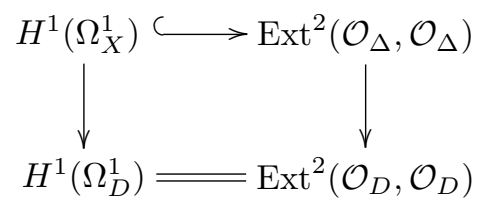

commutes, where the vertical arrows are restriction to $D$ and the Fourier-Mukai action, respectively.

Proof. Working through the Fourier-Mukai transform this is basically the statement that the induced map on normal bundles $\nu_{\Delta} \rightarrow \nu_{D \times X}$ (on the intersection $\Delta_{D}$ ) gives, after contraction with $\theta$, the restriction map $\Omega_{X}^{1} \rightarrow \Omega_{D}^{1}$ on cotangent bundles.

More explicitly, recall (90) that a class $\sigma \in H^{1}\left(\Omega_{X}^{1}\right)$ gives rise to an extension $0 \rightarrow$ $\mathcal{O}_{X} \rightarrow \mathcal{F}_{\sigma} \rightarrow T_{X} \rightarrow 0$ (85) which can be spliced together with $0 \rightarrow \Omega_{\Delta}^{1} \rightarrow \mathcal{O}_{2 \Delta} \rightarrow$ $\mathcal{O}_{\Delta} \rightarrow 0$ (89) by pushing the former forward under the diagonal map, and identifying $T_{X}$ with $\Omega_{\Delta}^{1}$ using $\theta$. This gives $[\sigma] \in \operatorname{Ext}^{2}\left(\mathcal{O}_{\Delta}, \mathcal{O}_{\Delta}\right)$.

Applying this as a Fourier-Mukai transform to $\mathcal{O}_{D}$ means taking the derived tensor product of it with $\mathcal{O}_{D \times X}$ and pushing down to the second $X$ factor. Since $D \times X$ is transverse to $\Delta$ we take the usual tensor product, yielding the splicing together of the exact sequences

$$
\left.\left.0 \rightarrow \mathcal{O}_{D} \rightarrow \mathcal{F}_{\sigma}\right|_{D} \rightarrow T_{X}\right|_{D} \rightarrow 0
$$

(pushed forward by $\Delta_{D} \subset X \times X$ ), and

$$
\left.0 \rightarrow \Omega_{\Delta}^{1}\right|_{D} \rightarrow \mathcal{O}_{2 \Delta_{D} \subset D \times X} \rightarrow \mathcal{O}_{\Delta_{D}} \rightarrow 0 .
$$

The central sheaf above denotes the (pushforward to $X \times X$ of the) thickening of $\Delta_{D}$ inside $D \times X$. 
Now in the first of the above exact sequences (91), taking the kernel of the surjections of the last two terms to $\nu_{D}$ gives

$$
0 \rightarrow \mathcal{O}_{D} \rightarrow \mathcal{F}_{\left.\sigma\right|_{D}} \rightarrow T_{D} \rightarrow 0
$$

where $\mathcal{F}_{\left.\sigma\right|_{D}}$ is the sheaf on $D$ given by the extension

$$
\left.\sigma\right|_{D} \in H^{1}\left(\Omega_{D}^{1}\right)=\operatorname{Ext}^{1}\left(T_{D}, \mathcal{O}_{D}\right)
$$

In the second (92), this surjection corresponds, under $\theta$, to $\left.\Omega_{\Delta}^{1}\right|_{D} \rightarrow \Omega_{\Delta_{D}}^{1}$. This does not lift to a map from the second term of (92) to $\Omega_{\Delta_{D}}^{1}$, but it does once we pushdown to the second $X$ factor. That is, the pushdown of (92) to $X$ gives

$$
\left.0 \rightarrow \Omega_{X}^{1}\right|_{D} \rightarrow J_{X}^{1}\left(\mathcal{O}_{D}\right) \rightarrow \mathcal{O}_{D} \rightarrow 0,
$$

where $J_{X}^{1}\left(\mathcal{O}_{D}\right)$ is the first jet space on $X$ of $\mathcal{O}_{D}$ (which is, by definition, the FourierMukai transform of $\mathcal{O}_{D}$ by $\left.\mathcal{O}_{2 \Delta}\right) . J_{X}^{1}\left(\mathcal{O}_{D}\right)$ surjects onto (the pushforward to $X$ of) $J_{D}^{1}\left(\mathcal{O}_{D}\right)$ (since $2 \Delta$ surjects onto the pushforward to $X \times X$ of $2 \Delta_{D} \subset D \times D$ ) and the latter splits as $\mathcal{O}_{D} \oplus \Omega_{D}^{1}$ since the Atiyah class on $D$ of $\mathcal{O}_{D}$ vanishes - the trivial line bundle on $D$ clearly admits a holomorphic connection. The resulting map $J_{X}^{1}\left(\mathcal{O}_{D}\right) \rightarrow \Omega_{D}^{1}$ induces via (94) the standard surjection $\left.\Omega_{X}^{1}\right|_{D} \rightarrow \Omega_{D}^{1}$, and taking the kernels of these surjections to $\Omega_{D}^{1}$ turns (94) into

$$
0 \rightarrow \nu_{D}^{*} \rightarrow \mathcal{O}_{2 D} \rightarrow \mathcal{O}_{D} \rightarrow 0 .
$$

Thus the pushdowns of (91) and (92) both have commuting surjections to $\Omega_{D}^{1} \cong \nu_{D}$, so their spliced representative of $\operatorname{Ext}^{2}\left(\mathcal{O}_{D}, \mathcal{O}_{D}\right)$ has a map to the trivial complex $\left\{\Omega_{D}^{1} \stackrel{\text { id }}{\rightarrow} \Omega_{D}^{1}\right\}$; taking kernels shows that it is quasi-isomorphic to the splicing of (93) and (95) via the isomorphism $\theta: T_{D} \cong \nu_{D}^{*}$. But this is precisely the sequence

$$
0 \rightarrow \mathcal{O}_{D} \rightarrow \mathcal{F}_{\left.\sigma\right|_{D}} \rightarrow \mathcal{O}_{2 D} \rightarrow \mathcal{O}_{D} \rightarrow 0
$$

defining $\left[\left.\sigma\right|_{D}\right] \in H^{1}\left(\Omega_{D}^{1}\right)=\operatorname{Ext}^{2}\left(\mathcal{O}_{D}, \mathcal{O}_{D}\right)$.

So everything is governed by intersection numbers. Let

$$
C_{i j} \stackrel{\text { def }}{=} C_{i} \cdot C_{j}=\left\{\begin{array}{cl}
-2 & i=j, \\
1 & |i-j|=1 \\
0 & |i-j|>1
\end{array}\right.
$$

be the $(n \times n)$ intersection matrix of the $A_{n}$-chain of curves $C_{i}$, with inverse $D_{i j}$. In fact one can calculate that

$$
D_{i j}=\frac{-1}{n+1} \min (i, j)(n-\max (i, j)+1) .
$$

Thus the classes $D_{i} \stackrel{\text { def }}{=} \sum_{i=1}^{n} D_{i j}\left[C_{j}\right] \in H^{1}\left(\Omega_{X}^{1}\right)$ are the dual basis to the $C_{i}$ for the span of the $C_{i}$ in $H^{1}\left(\Omega_{X}^{1}\right)$ under the intersection pairing (96): $D_{i} \cdot C_{j}=\delta_{i j}$.

We are finally ready to define the extra natural transformations id $\rightarrow \mathrm{id}[2]$ that we need to extend the braid group action to a representation of the braid cobordism category. 
Definition 5.4. Let

$$
X_{i}: \mathcal{O}_{\Delta} \rightarrow \mathcal{O}_{\Delta}[2]
$$

be defined by the above dual basis $D_{i} \in H^{1}\left(\Omega_{X}^{1}\right) \subset \operatorname{Ext}^{2}\left(\mathcal{O}_{\Delta}, \mathcal{O}_{\Delta}\right)$. That is, $X_{i}$ $=\sum_{i=1}^{n} D_{i j}\left[C_{j}\right]$.

For any $\mathcal{F} \in D^{b}(X)$ we also let $X_{i}: \mathcal{F} \rightarrow \mathcal{F}[2]$ denote the morphism induced by (98) by Fourier-Mukai transform.

For a proper irreducible curve $C \subset X$ let $\theta_{C}: \mathcal{O}_{C} \rightarrow \mathcal{O}_{C}[2]$ denote the generator corresponding to 1 in $\operatorname{Ext}^{2}\left(\mathcal{O}_{C}, \mathcal{O}_{C}\right) \cong H^{1,1}(C) \cong \mathbb{C}$.

Proposition 5.5. The morphisms $X_{i}: \mathcal{O}_{C_{j}} \rightarrow \mathcal{O}_{C_{j}}[2]$ are 0 for $i \neq j$ and $\theta_{j}$ for $j=i$. There are isomorphisms

$$
\begin{aligned}
& \mathrm{ev}_{i} \circ \mathrm{ev}_{i}^{\prime} \cong X_{i-1}-2 X_{i}+X_{i+1}: \mathcal{O}_{\Delta} \rightarrow \mathcal{O}_{\Delta}, \\
& \mathrm{ev}_{i}^{\prime} \circ \mathrm{ev}_{i} \cong X_{i} \bigotimes \mathrm{id}-\mathrm{id} \otimes X_{i}: U_{i} \rightarrow U_{i}[2] .
\end{aligned}
$$

Remarks. Here we have set $X_{0}=0=X_{n+1}$, and $\operatorname{id} \otimes X_{i}$ (for instance) maps $\mathcal{F} \otimes \mathcal{G} \rightarrow \mathcal{F} \otimes \mathcal{G}[2]$ in $D^{b}(X \times X)$ by the identity on $\mathcal{F}$ and $X_{i}$ on $\mathcal{G}$. Since $U_{i}=$ $\mathcal{O}_{C_{i} \times C_{i}}\left(C_{i}, 0\right)[-1]$ is of the form $\mathcal{F} \otimes \mathcal{G}$ this explains the notation above.

Proof. The first statement follows directly from Lemma 5.3 and the definition of the $D_{i}$. Since $\left[C_{i}\right]=D_{i-1}-2 D_{i}+D_{i+1} \in H^{1}\left(\Omega_{X}^{1}\right)$ by construction, the second statement is just a rephrasing of Proposition 5.2.

So it remains to prove the last isomorphism. Splicing together (79) and (80) (in the opposite order from before) gives the following exact sequence representing the morphism $\mathrm{ev}_{i}^{\prime} \circ \mathrm{ev}_{i}: \mathcal{O}_{C_{i} \times C_{i}}\left(C_{i}, 0\right) \rightarrow \mathcal{O}_{C_{i} \times C_{i}}\left(C_{i}, 0\right)[2]:$

$$
\begin{aligned}
0 & \rightarrow \mathcal{O}_{C_{i} \times C_{i}}\left(C_{i}, 0\right) \\
& \rightarrow \mathcal{O}_{\Delta \cup\left(C_{i} \times C_{i}\right)}\left(L_{i}\right) \rightarrow \mathcal{O}_{\Delta \cup\left(C_{i} \times C_{i}\right)}\left(C_{i}, 0\right) \rightarrow \mathcal{O}_{C_{i} \times C_{i}}\left(C_{i}, 0\right) \rightarrow 0 .
\end{aligned}
$$

$\mathcal{O}_{\Delta}\left(-\Delta_{C_{i}}\right)$ injects into the central two terms; taking their cokernels shows that $\left\{\mathrm{ev}_{i}^{\prime} \circ \mathrm{ev}_{i}: U_{i} \rightarrow U_{i}[2]\right\}$ is quasi-isomorphic to

$$
\left\{\mathcal{O}_{C_{i} \times C_{i}}\left(C_{i}, 0\right)\left(\Delta_{C_{i}}\right) \rightarrow \mathcal{O}_{2 \Delta_{C_{i}} \cup\left(C_{i} \times C_{i}\right)}\left(C_{i}, 0\right)\right\} .
$$

Here $2 \Delta_{C_{i}}$ is the thickening of $\Delta_{C_{i}}$ inside $C_{i} \times C_{i}$, and the central map above is the restriction map to $\mathcal{O}_{\Delta_{C_{i}}}\left(C_{i}, 0\right)\left(\Delta_{C_{i}}\right) \cong \mathcal{O}_{\Delta_{C_{i}}} \cong \mathcal{I}_{\Delta_{C_{i}} \subset 2 \Delta_{C_{i}}}\left(C_{i}, 0\right)$.

Now $\operatorname{Ext}^{2}\left(\mathcal{O}_{C_{i} \times C_{i}}\left(C_{i}, 0\right), \mathcal{O}_{C_{i} \times C_{i}}\left(C_{i}, 0\right)\right)=\operatorname{Ext}^{2}\left(\mathcal{O}_{C_{i} \times C_{i}}, \mathcal{O}_{C_{i} \times C_{i}}\right)$ is isomorphic to $H^{1}\left(\nu_{C_{i} \times C_{i}}\right) \cong H^{1}\left(\Omega_{C_{i} \times C_{i}}^{1}\right) \cong H^{1}\left(\Omega_{C_{i}}^{1}\right) \oplus H^{1}\left(\Omega_{C_{i}}^{1}\right)$, with the penultimate isomorphism given by contraction with the two form $\theta_{1}+\theta_{2}$, where $\theta_{j}$ is the pullback of $\theta$ from the $j$-th factor of $X \times X$. Under this isomorphism it is clear that the classes $D_{i} \oplus 0$ and $0 \oplus D_{i}$ correspond to $X_{i} \otimes$ id and id $\otimes X_{i}$ respectively.

These classes are, as usual, described the (pushforward from $C_{i} \times C_{i}$ to $X \times X$ of the) extension class $0 \rightarrow \mathcal{O}_{C_{i} \times C_{i}} \rightarrow \mathcal{F} \rightarrow T_{C_{i} \times C_{i}} \rightarrow 0$ corresponding to an element of $H^{1}\left(\Omega_{C_{i} \times C_{i}}^{1}\right)$, composed with the universal extension $0 \rightarrow \nu_{C_{i} \times C_{i}}^{*} \rightarrow \mathcal{O}_{2\left(C_{i} \times C_{i}\right)}$ $\rightarrow \mathcal{O}_{C_{i} \times C_{i}} \rightarrow 0$ by using $\theta_{1}+\theta_{2}$ to identify $T_{C_{i} \times C_{i}} \cong \nu_{C_{i} \times C_{i}}^{*}$. That is, these classes 
$\mathcal{O}_{C_{i} \times C_{i}} \rightarrow \mathcal{O}_{C_{i} \times C_{i}}[2]$ are quasi-isomorphic to

$$
\left\{\mathcal{F} \rightarrow \mathcal{O}_{2\left(C_{i} \times C_{i}\right)}\right\}
$$

If the $H^{1}\left(\Omega_{C_{i} \times C_{i}}^{1}\right)$ class is the fundamental class $[D]$ of an effective divisor $D \subset$ $C_{i} \times C_{i}$ then we have seen that we can describe $\mathcal{F}$ as $T_{C_{i} \times C_{i}} \times{ }_{\nu_{D}} \mathcal{O}_{C_{i} \times C_{i}}(D)(86)$. Then we divide both terms in $(100)$ by $T_{C_{i} \times C_{i}}(-\log D)$ and tensor with $\mathcal{O}\left(C_{i}, 0\right)$ to show that the morphism $[D]: \mathcal{O}_{C_{i} \times C_{i}}\left(C_{i}, 0\right) \rightarrow \mathcal{O}_{C_{i} \times C_{i}}\left(C_{i}, 0\right)[2]$ is quasi-isomorphic to the complex

$$
\left\{\mathcal{O}_{C_{i} \times C_{i}}(D)\left(C_{i}, 0\right) \rightarrow \mathcal{O}_{\left(C_{i} \times C_{i}\right) \cup 2 D}\left(C_{i}, 0\right)\right\},
$$

where $2 D$ is the first order thickening of $D$ in the directions perpendicular to $T_{D}$ under $\theta_{1}+\theta_{2}$ (which contain one more direction other than those of $T_{C_{i} \times C_{i}}$ ).

Picking $D=\Delta_{C_{i}} \subset C_{i} \times C_{i}$ we get $\Delta_{C_{i}}$ thickened inside $\Delta$ in (101), which is not quite (99). But if we change the identification $T_{C_{i} \times C_{i}} \cong \nu_{C_{i} \times C_{i}}^{*}$ by using $\theta_{1}-\theta_{2}$ instead of $\theta_{1}+\theta_{2}$, then we get $D$ thickened in the directions perpendicular to $T_{D}$ under $\theta_{1}-\theta_{2}$ in (101). This now describes the Ext ${ }^{2}$ class corresponding not to $[D] \in H^{1}\left(\Omega_{C_{i} \times C_{i}}^{1}\right)$ but to its image under the map multiplying its second component in its Künneth decomposition by -1 .

Applying this to $D=\Delta_{C_{i}}$, whose class in $H^{1}\left(\Omega_{C_{i} \times C_{i}}^{1}\right)$ has degree 1 on both factors, proves that the Ext ${ }^{2}$ class described by the cohomology class $\left(D_{i},-D_{i}\right)$ corresponds to the complex (101) with $D=\Delta_{C_{i}}$ thickened inside $C_{i} \times C_{i}$. But this is precisely $(99)$, so that $\left(X_{i} \otimes \mathrm{id}\right)-\left(\mathrm{id} \otimes X_{i}\right) \cong \mathrm{ev}_{i}^{\prime} \circ \mathrm{ev}_{i}$.

Given a kernel $K \in D^{b}(X \times X)$ we denote by $l_{X_{i}}: K \rightarrow K[2]$ the following morphism (giving rise to a natural transformation between the Fourier-Mukai functors associated to $K$ and $K[2])$ :

$$
K \cong \mathcal{O}_{\Delta} \star K \stackrel{X_{i} \star \mathrm{id}}{\longrightarrow} \mathcal{O}_{\Delta}[2] \star K \cong K[2] .
$$

Similarly $r_{X_{i}}: K \rightarrow K[2]$ is

$$
K \cong K \star \mathcal{O}_{\Delta} \stackrel{\text { id } \star X_{i}}{\longrightarrow} K \star \mathcal{O}_{\Delta}[2] \cong K[2] .
$$

It is clear that on $\mathcal{O}_{\Delta}, l_{X_{i}}$ and $r_{X_{i}}$ act as the same morphism. Given a linear combination $a=\sum_{j} a_{j} X_{j}$ of the $X_{i}$ we also set

$$
l_{a} \stackrel{\text { def }}{=} \sum_{j} a_{j} l_{X_{j}} \quad \text { and } \quad r_{a} \stackrel{\text { def }}{=} \sum_{j} a_{j} r_{X_{j}} .
$$

Then we have the following analogue of Proposition 3.4.

Proposition 5.6. The morphisms $T_{i} \rightarrow T_{i}[2]$ and $T_{i}^{\prime} \rightarrow T_{i}^{\prime}[2]$ given by

$$
l_{a}-r_{a+a_{i}\left(X_{i-1}-2 X_{i}+X_{i+1}\right)}
$$

are both zero. For instance for $i \neq j, l_{X_{j}}$ and $r_{X_{j}}$ act as isomorphic morphisms on $T_{i}$ and as zero on $U_{i}$. 
Homology, Homotopy and Applications, vol. 9(2), 2007

Proof. We first claim that on $U_{i}=\mathcal{O}_{C_{i}}\left(C_{i}\right)[-1] \otimes \mathcal{O}_{C_{i}}$, the functors $l_{X_{i}}$ and $r_{X_{i}}$ act as id $\otimes X_{i}$ and $-X_{i} \otimes$ id respectively. For instance $r_{X_{i}}$ acts as

$$
U_{i} \star \mathcal{O}_{\Delta} \stackrel{\operatorname{id} \star X_{i}}{\longrightarrow} U_{i} \star \mathcal{O}_{\Delta}[2]
$$

which is, in the usual notation of Section 4.2 (cf. (25)),

$$
\pi_{13 *}\left[\mathcal{O}_{\Delta \times X} \otimes \mathcal{O}_{X \times C_{i} \times C_{i}}\left(0, C_{i}, 0\right)[-1] \stackrel{\pi_{12}^{*} X_{i} \otimes \mathrm{id}}{\longrightarrow} \mathcal{O}_{\Delta \times X} \otimes \mathcal{O}_{X \times C_{i} \times C_{i}}\left(0, C_{i}, 0\right)[1]\right]
$$

which is

$$
\pi_{13 *}\left[\mathcal{O}_{\Delta_{C_{i}} \times C_{i}}\left(0, C_{i}, 0\right)[-1] \longrightarrow \mathcal{O}_{\Delta_{C_{i}} \times C_{i}}\left(0, C_{i}, 0\right)[1]\right] .
$$

Here the arrow is pulled back from the first and second factors, so the result is of the form

$$
\mathcal{O}_{C_{i} \times C_{i}}\left(C_{i}, 0\right)[-1] \stackrel{Z \bowtie i d}{\longrightarrow} \mathcal{O}_{C_{i} \times C_{i}}\left(C_{i}, 0\right)[1],
$$

where, by the above working, $Z$ is

$$
\pi_{1 *}\left[\mathcal{O}_{\Delta} \otimes \mathcal{O}_{X \times C_{i}}\left(0, C_{i}\right)[-1] \stackrel{X_{i} \otimes \mathrm{id}}{\longrightarrow} \mathcal{O}_{\Delta} \otimes \mathcal{O}_{X \times C_{i}}\left(0, C_{i}\right)[1]\right]
$$

This is where the sign comes in, as this is not $X_{i}: \mathcal{O}_{C_{i}}\left(C_{i}\right)[-1] \rightarrow \mathcal{O}_{C_{i}}\left(C_{i}\right)[1]$, the latter being by definition the map of Fourier-Mukai transforms

$$
\Phi_{\mathcal{O}_{\Delta}}\left(\mathcal{O}_{C_{i}}\left(C_{i}\right)[-1]\right) \stackrel{\Phi_{X_{i}}}{\longrightarrow} \Phi_{\mathcal{O}_{\Delta}[2]}\left(\mathcal{O}_{C_{i}}\left(C_{i}\right)[-1]\right),
$$

which is

$$
\pi_{2 *}\left[\mathcal{O}_{\Delta} \otimes \mathcal{O}_{C_{i} \times X}\left(C_{i}, 0\right)[-1] \stackrel{X_{i} \otimes \mathrm{id}}{\longrightarrow} \mathcal{O}_{\Delta} \otimes \mathcal{O}_{C_{i} \times X}\left(C_{i}, 0\right)[1]\right]
$$

Comparing with (104), the two differ just by swapping the two factors of $X \times X$, which sends $X_{i}$ to $-X_{i}$ because it reverses the identification of the normal bundle of $\Delta$ with $T_{\Delta}$ (83).

For $l_{X_{i}}$ the working is the same but easier, without the swapping of the $X \times X$ factors.

Now $l_{a}$ acts on $T_{i}$ as

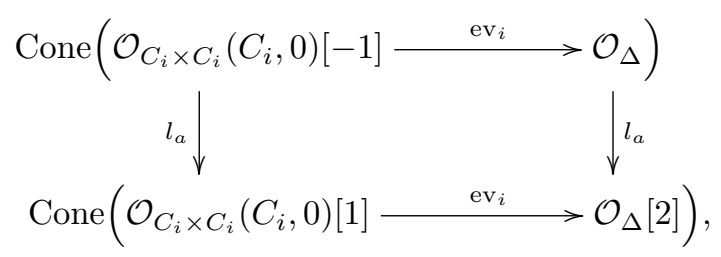


Homology, Homotopy and Applications, vol. 9(2), 2007

which, by the above and the first part of Proposition 5.5 is

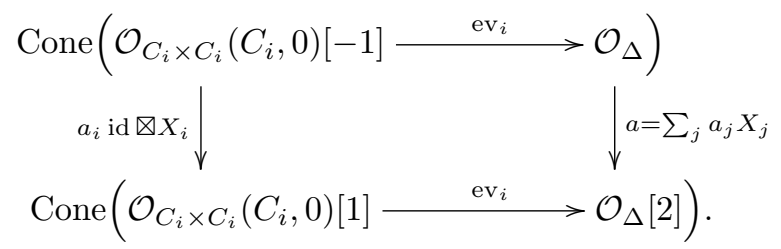

Now use the homotopy $h$ which maps the top right corner to the bottom left by $\mathrm{ev}_{i}^{\prime}$, so that $d h+h d$ is $\mathrm{ev}_{i}^{\prime} \circ \mathrm{ev}_{i}$ on the left and $\mathrm{ev}_{i} \circ \mathrm{ev}_{i}^{\prime}$ on the right. Adding $a_{i}$ times this to the given maps and using Proposition 5.5, we find that $l_{a}: T_{i} \rightarrow T_{i}$ is homotopic to

$$
\begin{aligned}
& \text { Cone }\left(\mathcal{O}_{C_{i} \times C_{i}}\left(C_{i}, 0\right)[-1] \stackrel{\mathrm{ev}_{i}}{\longrightarrow} \mathcal{O}_{\Delta}\right) \\
& a_{i} \operatorname{id} \otimes X_{i}+a_{i}\left(X_{i} \otimes \mathrm{id}-\mathrm{id} \otimes X_{i}\right) \downarrow=a_{i} X_{i} \otimes \mathrm{id} \quad \downarrow a+a_{i}\left(X_{i-1}-2 X_{i}+X_{i+1}\right) \\
& \text { Cone }\left(\mathcal{O}_{C_{i} \times C_{i}}\left(C_{i}, 0\right)[1] \stackrel{\mathrm{ev}_{i}}{>} \mathcal{O}_{\Delta}[2]\right) \text {. }
\end{aligned}
$$

Since the coefficient of $X_{i}$ in $a+a_{i}\left(X_{i-1}-2 X_{i}+X_{i+1}\right)$ is $-a_{i}$, this is

$$
r_{a+a_{i}\left(X_{i-1}-2 X_{i}+X_{i+1}\right)}: T_{i} \rightarrow T_{i} .
$$

The proof for $T_{i}^{\prime}$ is almost identical, using $\mathrm{ev}_{i}$ in the homotopy.

We define the morphisms (between Fourier-Mukai kernels, inducing natural transformations between the corresponding functors) associated to positive and negative braid moves as follows.

For a positive braid move we define the morphisms

$$
\mathcal{O}_{\Delta} \rightarrow T_{i} \quad \text { and } \quad T_{i}^{\prime} \rightarrow \mathcal{O}_{\Delta}
$$

by the first morphism of (79) and the second morphism of (80) respectively.

For the negative braid moves we write $T_{i}=\mathcal{O}_{\Delta \cup\left(C_{i} \times C_{i}\right)}\left(C_{i}, 0\right)$ as the cone on $\mathcal{O}_{C_{i} \times C_{i}}\left(C_{i}, 0\right)[-1] \stackrel{\mathrm{ev}_{i}}{\longrightarrow} \mathcal{O}_{\Delta}$ by $(79)$, and then define $\delta_{i}: T_{i} \rightarrow \mathcal{O}_{\Delta}[2]$ by

$$
\begin{aligned}
\text { Cone }\left(\mathcal{O}_{C_{i} \times C_{i}}\left(C_{i}, 0\right)[-1] \stackrel{\mathrm{ev}_{i}}{\rightarrow}\right. & \left.\mathcal{O}_{\Delta}\right) \\
& \downarrow X_{i+1}-X_{i-1} \\
\downarrow & \mathcal{O}_{\Delta}[2] .
\end{aligned}
$$

To check this really defines a morphism in $D^{b}(X \times X)$ we need to know that

$$
\left(X_{i-1}-X_{i+1}\right) \circ \mathrm{ev}_{i} \cong 0 \text {. }
$$

In fact we claim that

$$
\operatorname{Hom}^{0}\left(\mathcal{O}_{C_{i} \times C_{i}}\left(C_{i}, 0\right)[-1], \mathcal{O}_{\Delta}[2]\right)=0 .
$$

To prove (107), note that $C_{i} \times C_{i}$ and $\Delta$ intersect with excess dimension one and excess normal bundle $\mathcal{O}_{\Delta_{C_{i}}}\left(C_{i}\right)$. Therefore a standard Koszul resolution argument 
shows that the only nonzero $\mathcal{E} x t^{i}$ is

$$
\mathcal{E} x t^{1}\left(\mathcal{O}_{C_{i} \times C_{i}}\left(C_{i}, 0\right), \mathcal{O}_{\Delta}\right) \cong \mathcal{O}_{\Delta_{C_{i}}},
$$

generated by $\mathrm{ev}_{i}$. So the local-to-global spectral sequence $H^{i}\left(\mathcal{E} x t^{j}\right) \Longrightarrow \operatorname{Ext}^{i+j}$ shows that $\operatorname{Ext}^{3}\left(\mathcal{O}_{C_{i} \times C_{i}}\left(C_{i}, 0\right), \mathcal{O}_{\Delta}\right)=0$.

Similarly, writing $T_{i}^{\prime}$ as the cone on $\mathcal{O}_{\Delta}[-1] \stackrel{\mathrm{ev}_{i}^{\prime}}{\longrightarrow} \mathcal{O}_{C_{i} \times C_{i}}\left(C_{i}, 0\right)$ by (80), then a chase around the diagram proving that $T_{i}^{\prime} \star T_{i} \cong \mathcal{O}_{\Delta}$ (i.e. a proof of braid movie move 12 , which will be given below) shows that the corresponding morphism $\delta_{i}: \mathcal{O}_{\Delta}[-2] \rightarrow T_{i}^{\prime}$ is

$$
\begin{aligned}
& X_{i+1}-X_{i-1} \mathcal{O}_{\Delta}[-3] \\
& \operatorname{Cone}\left(\mathcal{O}_{\Delta}[-1] \stackrel{\mathrm{ev}^{\prime}}{\longrightarrow} \mathcal{O}_{C_{i} \times C_{i}}\left(C_{i}, 0\right)\right) .
\end{aligned}
$$

Theorem 5.7. The $T_{i}, T_{i}^{\prime}$ and morphisms described above induce a projective action of the braid cobordism category on $D^{b}(X)$.

Proof. The braid relations were already proved in $[\mathbf{S T}]$. For the first 10 braid movie moves of Section 3.1 we use the same trick as in the proof of Theorem 1.1 in Section 3.2. They each describe two isomorphisms between the same two FourierMukai kernels (a combination of $T_{i} \mathrm{~s}$ and $T_{j}^{\prime} \mathrm{s}$ ), and we would like to show that these isomorphisms are projectively equivalent. Composing one with the inverse of the other (via the operation $\star$ of $(25)$ ), we get an isomorphism from one kernel to itself which we are required to show is a scalar. But the kernel is invertible, so composing with its inverse we get an isomorphism $\mathcal{O}_{\Delta} \rightarrow \mathcal{O}_{\Delta}$, i.e. an invertible element of $\operatorname{Hom}^{0}\left(\mathcal{O}_{\Delta}, \mathcal{O}_{\Delta}\right) \cong H^{0}\left(\mathcal{O}_{X}\right)$. This must be a nonzero scalar by the Nullstellensatz.

For the moves 11-15 with one positive branch point, we use the fact that $\operatorname{Hom}^{0}\left(T_{i}^{\prime}, \mathcal{O}_{\Delta}\right) \cong \mathbb{C} \cong \operatorname{Hom}^{0}\left(\mathcal{O}_{\Delta}, T_{i}\right)$ with generators the second morphism of (80) and the first morphism of (79), respectively. This is proved in much the same way as one computes the other degree parts of (107) (or can be deduced from it). Namely (108) shows that the morphisms from $U_{i}$ to $\mathcal{O}_{\Delta}$ are one-dimensional, generated by $\mathrm{ev}_{i}$. A long exact sequence then gives the result we want.

By composing with invertible Fourier-Mukai kernels we find that the morphisms from a kernel at the top of any of the movies 11-14 to the one at the bottom are also one dimensional and generated by the morphism represented by the movie. Therefore under a braid movie move this can only change by an invertible scalar.

Next we deal with move 11 with one negative branch point using the map $\delta_{j}$ (109) by showing that, for instance, for $|i-j|>1$ the composition

$$
T_{i} \cong T_{i} \star \mathcal{O}_{\Delta} \stackrel{\delta_{j}}{\longrightarrow} T_{i} \star T_{j}^{\prime}[2] \cong T_{j}^{\prime} \star T_{i}[2]
$$

is the same as the composition

$$
T_{i} \cong \mathcal{O}_{\Delta} \star T_{i} \stackrel{\delta_{j}}{\longrightarrow} T_{j}^{\prime} \star T_{i}[2] .
$$

However the first composition (110) is the map $r_{j+1}-r_{j-1}$ of the cone $U_{i} \stackrel{\mathrm{ev}_{i}}{\longrightarrow} \mathcal{O}_{\Delta}$ 
into the top line of the cone

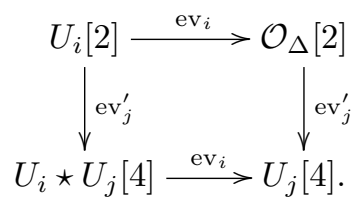

Since this map is zero on the $U_{i}$ piece (by the last part of Proposition 5.6) and equal to $l_{j-1}-l_{j+1}$ on $\mathcal{O}_{\Delta}$, and since $U_{i} \star U_{j} \cong 0 \cong U_{j} \star U_{i}$ for $|i-j|>1$, we find it is isomorphic to the map $l_{j-1}-l_{j+1}$ of the cone $U_{i} \stackrel{\mathrm{ev}_{i}}{\longrightarrow} \mathcal{O}_{\Delta}$ into the top line of the cone

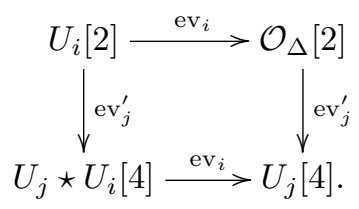

Reflecting this in its diagonal gives the second composition (111).

For move 12 with one negative branch point we recall the quasi-isomorphism that makes $T_{i} \star T_{i}^{\prime} \cong \mathrm{id}$. (This is given in $[\mathbf{S T}]$ using functors, but that proof can be easily translated into Fourier-Mukai transforms, and takes the exact same form as our proof in Proposition 4.6 since the $U_{i}$ s satisfy the same relations.) Namely $U_{i} \star U_{i} \cong U_{i}[-2] \oplus U_{i}$, and $T_{i} \star T_{i}^{\prime}$ is the total cone on

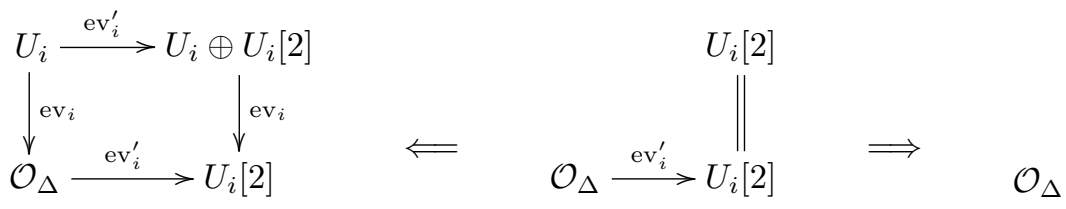

with the double arrows the obvious quasi-isomorphisms. Then move 12 says that the morphism from the first square to the cone $\left(\mathcal{O}_{\Delta}[2] \stackrel{\mathrm{ev}_{i}^{\prime}}{\longrightarrow} U_{i}[4]\right)$ given by zero on the top row and $r_{X_{i-1}-X_{i+1}}$ applied to both terms on the bottom row should be isomorphic to the morphism from the last square (i.e. just $\mathcal{O}_{\Delta}$ ) to the cone $\left(\mathcal{O}_{\Delta}[2] \stackrel{\mathrm{ev}_{i}^{\prime}}{\longrightarrow} U_{i}[4]\right)$ given by $r_{X_{i-1}-X_{i+1}}$ from $\mathcal{O}_{\Delta}$ to $\mathcal{O}_{\Delta}[2]$. But indeed both are isomorphic to the morphism from the central square to the cone $\left(\mathcal{O}_{\Delta}[2] \stackrel{\mathrm{ev}_{i}^{\prime}}{\longrightarrow} U_{i}[4]\right)$ given by zero on the top row and $X_{i-1}-X_{i+1}$ applied to both terms on the bottom row.

Move 13 we defer to the proofs for $D^{b}\left(T^{*} F l\right)$ in the next section; this paper is quite long enough without repeating proofs twice, so we concentrate on the harder $T^{*} \mathrm{Fl}$ case - for surfaces the same proof applies but is much easier since there is no $U_{i j}$ term involved in proving Theorem 4.12. As we noted before, move 14 follows from the various versions of move 13 .

Finally locality moves 15 with one negative branch point follow from the second part of Proposition 5.6; we describe how in the example of move 15 drawn in Figure 7 . For that we combine the morphism $\delta_{k}: T_{k} \rightarrow \mathcal{O}_{\Delta}[2]$ of (106) and a standard 
isomorphism $\phi$ of Fourier-Mukai kernels $K \stackrel{\phi}{\rightarrow} K^{\prime}$ (a composition of one of the braid relations) consisting of cones of morphisms of kernels with expressions $U_{i} \star \ldots \star U_{j}$ involving only $U_{i}$ s with $|i-k|>1$ (and one copy of $\mathcal{O}_{\Delta}$, on which $\phi$ acts as the identity). We would like to show that the compositions of the following vertical maps of horizontal cones

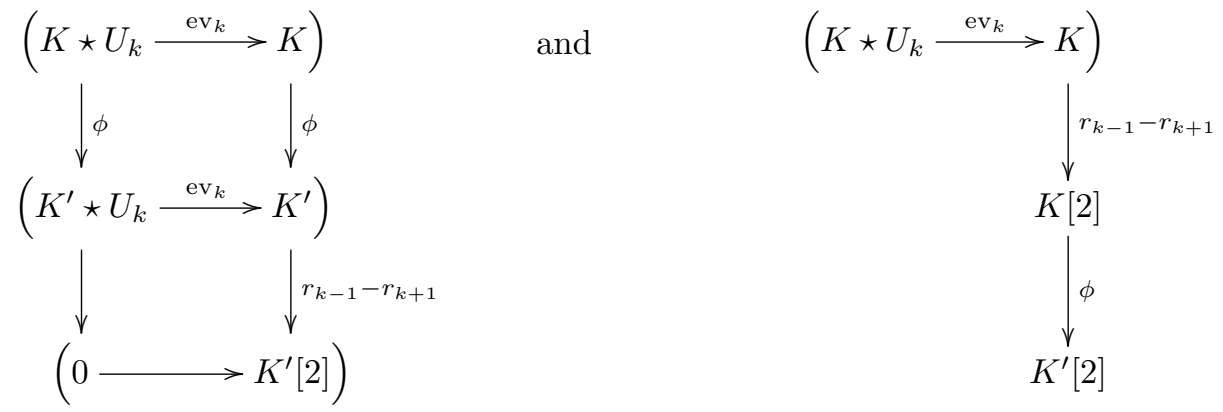

are the same, which is the case if the following compositions are equal:

$$
K \stackrel{\phi}{\longrightarrow} K^{\prime} \stackrel{r_{k-1}-r_{k+1}}{\longrightarrow} K^{\prime}[2] \quad \text { and } \quad K \stackrel{r_{k-1}-r_{k+1}}{\longrightarrow} K[2] \stackrel{\phi}{\longrightarrow} K^{\prime}[2] \text {. }
$$

But by Proposition 5.6, $r_{k-1}-r_{k+1}$ acts as zero on every term in $K$ and $K^{\prime}$ except for the $\mathcal{O}_{\Delta}$ term in each, on which $\phi$ acts as the identity and so commutes with $r_{k-1}-r_{k+1}$.

One can show that the central ambiguity in the projective action of the braid cobordism category is at most $\pm 1 \subset \mathbb{C}^{*}$, by passing from $D^{b}(X)$ to the homotopy category $\mathcal{C}\left(A_{n} \otimes \mathbb{C}\right)$ of Section 2 , as described at the beginning of this Section. Under the equivalence $[\mathbf{K S}, \mathbf{S T}]$ of the derived categories of differential graded modules over $\operatorname{RHom}\left(\oplus_{i} \mathcal{O}_{C_{i}}, \oplus_{i} \mathcal{O}_{C_{i}}\right)$ and $A_{n} \otimes \mathbb{C}$, the $\mathcal{O}_{C_{i}}$ are mapped to the $P_{i}, T_{i}$ to $R_{i}$, $T_{i}^{\prime}$ to $R_{i}^{\prime}$, and $\mathrm{ev}_{i}, \mathrm{ev}_{i}^{\prime}$ to $\beta_{i}, \gamma_{i}$. The rational numbers $D_{i j}(97)$ that we used become integers under this equivalence, since by Proposition 5.5 the $X_{i}$ act on the $\mathcal{O}_{C_{i}}$ by integral generators of $H^{1,1}\left(C_{j}\right) \cong \operatorname{Ext}^{2}\left(\mathcal{O}_{C_{j}}, \mathcal{O}_{C_{j}}\right)$.

The subcategory generated by the projective modules $P_{i}$ is then equivalent to $\mathcal{C}\left(A_{n} \otimes \mathbb{C}\right)$, and the above positive $(105)$ and negative $(106)$ braid moves map to those of $(10,11)$ and $(12,13)$ respectively: for the positive braid moves this is obvious, while for the negative ones it follows from Proposition 5.5. Therefore the $\mathbb{C}^{*}$ scalar ambiguities in the movie moves in $D^{b}(X)$ map to the scalar ambiguities \pm 1 in $\mathcal{C}\left(A_{n} \otimes \mathbb{C}\right)$, and so are themselves \pm 1 . Of course we expect that they can all be shown to be +1 , but this would require more work.

\subsection{The cotangent bundle of the flag variety}

The construction of the last section goes over almost unchanged to $D^{b}\left(T^{*} F l\right)$; we outline the steps here. The kernels $U_{i}, T_{i}, T_{i}^{\prime}$ from Sections 4 and 5.1 are obviously analogous, as are the maps between them. Using the holomorphic symplectic structure of $K=T^{*} F l$ we find that $T_{K} \cong \Omega_{K}^{1}$, so that the description (82) of $\operatorname{Ext}^{2}\left(\mathcal{O}_{\Delta}, \mathcal{O}_{\Delta}\right)$ still holds. The divisors $N_{i} \subset K$ define de Rham classes $\left[N_{i}\right] \in$ $H^{1}\left(\Omega_{K}^{1}\right) \subset \operatorname{Ext}^{2}\left(\mathcal{O}_{\Delta}, \mathcal{O}_{\Delta}\right)$ by the same formulae as for the $\left[C_{i}\right]$ before (splicing the 
logarithmic one-forms sequence (84) to the doubled diagonal exact sequence (88) via $\left.T_{K} \cong \Omega_{K}^{1}\right)$.

Taking the same linear combinations $D_{i j}(97)$ of these classes as before we define the $X_{i} \in \operatorname{Ext}^{2}\left(\mathcal{O}_{\Delta}, \mathcal{O}_{\Delta}\right)$ as $\sum_{j} D_{i j}\left[N_{j}\right]$.

The analogue of Lemma 5.3 is that the action of such classes on some $\mathcal{O}_{N_{k}}$ by Fourier-Mukai transform is described by the restriction of the $H^{1}\left(\Omega_{K}^{1}\right)$ class to $H^{1}\left(\Omega_{N_{k} / K_{k}}^{1}\right) \cong H^{1}\left(\mathcal{O}_{N_{k}}\left(N_{k}\right)\right) \cong \operatorname{Ext}^{2}\left(\mathcal{O}_{N_{k}}, \mathcal{O}_{N_{k}}\right)$. This is proved in exactly the same way by noting that the natural map $T_{K} \rightarrow \mathcal{O}_{N_{k}}\left(N_{k}\right)$ becomes, on contraction with the holomorphic symplectic form, the restriction map $\Omega_{K}^{1} \rightarrow \Omega_{N_{k} / K_{k}}^{1}$. (The fact that $\operatorname{Ext}^{2}\left(\mathcal{O}_{N_{k}}, \mathcal{O}_{N_{k}}\right) \cong H^{1}\left(\mathcal{O}_{N_{k}}\left(N_{k}\right)\right)$ follows easily from the local-to-global spectral sequence for Ext and the Leray spectral sequence for $N_{k} \rightarrow K_{k}$.)

Now in turn, we can calculate $H^{1}\left(\Omega_{N_{k} / K_{k}}^{1}\right)$ by the Leray spectral sequence, using the fact that $R^{1} p_{k *} \Omega_{N_{k} / K_{k}}^{1} \cong \mathcal{O}_{K_{k}}$ by relative Serre duality, with all other derived functors zero. Therefore basechange holds, and the fibers of $p_{k}: N_{k} \rightarrow K_{k}$ are compact, so to determine a class in $H^{1}\left(\Omega_{N_{k} / K_{k}}^{1}\right) \cong H^{0}\left(\mathcal{O}_{K_{k}}\right)$ we need only determine its de Rham or singular cohomology class on each fiber, for instance by intersection theory.

In particular, for $|i-j|>1, N_{i} \cap N_{j}=N_{i j}$ is zero on the generic fiber of $N_{j} \rightarrow$ $K_{j}$ (being the pullback of the divisor $K_{i j} \subset K_{j}$ ) so $\left.\left[N_{i}\right]\right|_{N_{j}}$ defines the zero class in $H^{0}\left(\mathcal{O}_{K_{j}}\right)$. (So here it is crucial that the $\operatorname{Ext}^{2}\left(\mathcal{O}_{N_{j}}, \mathcal{O}_{N_{j}}\right)$ class depends only on the relative, fiberwise, $H^{2}$ class of $\left.\left[N_{i}\right]\right|_{N_{j}}$, rather its absolute class, which is nonzero.) For $i-j=1, N_{i} \cap N_{j}=N_{i j}$ is generically a section of $N_{j} \rightarrow K_{j}$ (away from $M_{i j} \subset N_{i j}$, by Lemma 4.8) and so defines the canonical generator $1 \in H^{0}\left(\mathcal{O}_{K_{j}}\right)$ away from $K_{i j} \subset K_{j}$, and so in fact everywhere by Hartog's theorem. Similarly, considering $i=j, N_{i}$ has degree -2 on each fiber of $N_{i} \rightarrow K_{i}$ so we find that $\left.\left[N_{i}\right]\right|_{N_{i}}$ is represented, via the Leray spectral sequence, by $-2 \in H^{0}\left(\mathcal{O}_{K_{i}}\right)$.

These numbers coincide, of course, with the intersections of the $C_{i}$ in Section 5.1. Therefore, by construction of the $D_{i j}$, (97) we find that the $X_{i}$ act as zero on $\mathcal{O}_{N_{j}}$ for $j \neq i$ and as the generator of $\operatorname{Ext}^{2}\left(\mathcal{O}_{N_{i}}, \mathcal{O}_{N_{i}}\right) \cong H^{0}\left(\mathcal{O}_{K_{i}}\right)$ on $\mathcal{O}_{N_{i}}$.

This gives the correct analogue of the first part of Proposition 5.5. The last part follows almost verbatim the proof for surfaces. For the second part the proof is also very similar, using relative de Rham or singular cohomology classes as above instead of absolute ones. It follows immediately that the corresponding $l_{X_{i}}$ and $r_{X_{i}}$ satisfy the same result as in Proposition 5.6.

With all of these technical results done, the proofs of the braid movie moves carry over just as before. For move 11 with one negative branch point we no longer have $U_{j} \star U_{i} \cong 0 \cong U_{i} \star U_{j}$ for $|i-j|>1$, but all we need is that they commute. But the proof of Proposition 4.7 shows that both $U_{i} \star U_{j}$ and $U_{j} \star U_{i}$ are isomorphic to $\mathcal{O}_{N_{i j} \times_{T^{*} F l_{i j}} N_{i j}}\left(D_{i j}\right)[-2]$ in the notation of $(50)$.

We have to prove the following version of move 13 with one negative branch 
point; that for $j=i+1$ the following two compositions
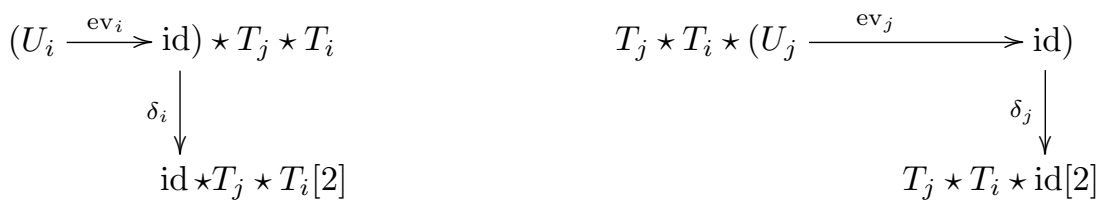

become isomorphic maps $T_{i} \star T_{j} \star T_{i} \rightarrow T_{j} \star T_{i}[2]$ upon identifying $T_{i} \star T_{j} \star T_{i} \cong$ $T_{j} \star T_{i} \star T_{j}$ by the second part of Theorem 4.12.

By the first part of Theorem 4.12 the isomorphism $T_{i} \star T_{j} \star T_{i} \cong T_{j} \star T_{i} \star T_{j}$ comes from an isomorphism $U_{i} \star T_{j} \star T_{i} \cong T_{j} \star T_{i} \star U_{j}$ of the first two terms in the two cones on the top line of the above diagram; as proved there this intertwines the two ev maps on the top line. Since these two terms map to zero in the bottom line, it remains to prove that the compositions

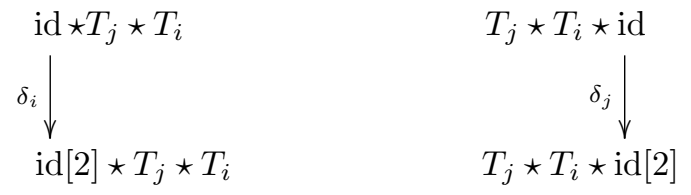

are equal. The left-hand one is $\left(l_{X_{i+1}}-l_{X_{i-1}}\right) \star \mathrm{id}=l_{X_{i+1}} \star \mathrm{id}$ by the last part of Proposition 5.6. By the first part of Proposition 5.6 this is $\left(r_{X_{i}}-r_{X_{i+1}}\right) \star \mathrm{id}=$ id $\star\left(l_{X_{i}}-l_{X_{i+1}}\right)$.

The right-hand map is $\mathrm{id} \star\left(r_{X_{j+1}}-r_{X_{j-1}}\right)=\mathrm{id} \star\left(-r_{X_{j-1}}\right)$ by the last part of Proposition 5.6. By the first part of Proposition 5.6 this is also id $\star\left(l_{X_{i}}-l_{X_{i+1}}\right)$.

The other negative versions of move 13 are similar; for instance showing that the following diagram becomes commutative

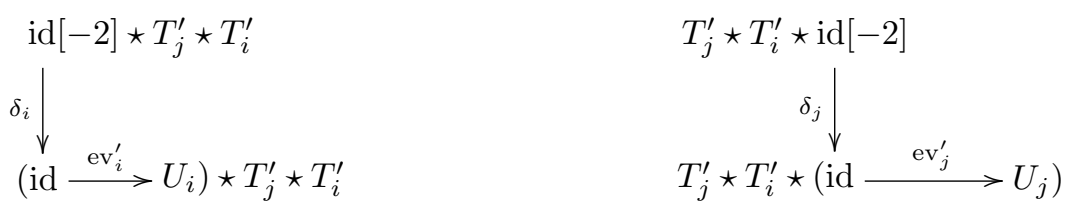

on mapping each $T_{j}^{\prime} \star T_{i}^{\prime}$ on the left-hand side by the identity to the corresponding $T_{j}^{\prime} \star T_{i}^{\prime}$ on the right-hand side, and mapping $U_{i} \star T_{j}^{\prime} \star T_{i}^{\prime}$ to $T_{j}^{\prime} \star T_{i}^{\prime} \star U_{j}$ by the isomorphism given by taking adjoints in Proposition 4.12. This commutes with maps on both sides by the adjoint of Proposition 4.12, so we are left with showing that the maps

$$
\left(l_{X_{i+1}}-l_{X_{i-1}}\right) \star \mathrm{id} \quad \text { and } \quad \mathrm{id} \star\left(r_{X_{j+1}}-r_{X_{j-1}}\right): T_{j}^{\prime} \star T_{i}^{\prime}[-2] \rightarrow T_{j}^{\prime} \star T_{i}^{\prime}
$$

are the same. But this follows as above by using Proposition 5.6.

\section{References}

[A] S. Ariki, On the decomposition numbers of the Hecke algebra of G(m,1,n), J. Math. Kyoto Univ. 36 (1996), 789-808. 
Homology, Homotopy and Applications, vol. 9(2), 2007

[BL] J. Baez and L. Langford, 2-Tangles, Lett. Math. Phys. 43 (1998), 187-197, arXiv:q-alg/9703033.

[BN] D. Bar-Natan, Khovanov's homology for tangles and cobordisms, Geom. Topol. 9 (2005) 1443-1499, arXiv:math.GT/0410495.

[BFK] J. Bernstein, I. Frenkel, and M. Khovanov, A categorification of the Temperley-Lieb algebra and Schur quotients of $U(\mathfrak{s l}(2))$ via projective and Zuckerman functors, Selecta Math. 5 (1999), 199-241, arXiv:math.QA/ 0002087.

[BG] J. Bernstein and S. Gelfand, Tensor products of finite- and infinitedimensional representations of semisimple Lie algebras, Compositio Math. 41 (1980), 245-285.

[BMR] R. Bezrukavnikov, I. Mirković, and D. Rumynin, Singular localization and intertwining functors for semisimple Lie algebras in prime characteristic, Ann. of Math., to appear, arXiv:math.RT/0602075.

[BO] A. Bondal and D. Orlov, Semiorthogonal decomposition for algebraic varieties, preprint, arXiv:alg-geom/9506012.

[Ca] A. Căldăraru, The Mukai pairing, I: the Hochschild structure, preprint, arXiv:math. AG/0308079.

[CS1] J. S. Carter and M. Saito, Braids and movies, J. Knot Theory Ramifications 5 (1996), 589-608.

[CS2] J. S. Carter and M. Saito, Knotted Surfaces and their Diagrams, Math. Surv. and Mon. 55, AMS, Providence, RI, 1998.

[CR] J. Chuang and R. Rouquier, Derived equivalences for symmetric groups and $\mathfrak{s l}_{2}$-categorification, Ann. of Math., to appear, arXiv:math.RT/ 0407205.

[De] P. Deligne, Action du groupe des tresses sur une catégorie, Invent. Math. 128 (1997), 159-175.

[FKS] I. Frenkel, M. Khovanov, and O. Schiffmann, Homological realization of Nakajima varieties and Weyl group actions, Compositio Mathematica 141 (2005), 1479-1503, arXiv:math.QA/0311485.

[FO3] K. Fukaya, Y.-G. Oh, H. Ohta, and K. Ono, Lagrangian Intersection Floer Theory - Anomaly and Obstruction, to appear, International Press.

[Ho] R. P. Horja, Derived category automorphisms from mirror symmetry, Duke Math. J. 127 (2005), 1-34, arXiv:math.AG/0103231.

[HK] R. S. Huerfano and M. Khovanov, A category for the adjoint representation, J. Algebra 246 (2001), 514-542, arXiv:math.QA/0002060.

[Hu] D. Huybrechts, Fourier-Mukai Transforms in Algebraic Geometry, Oxford Mathematical Monographs, Oxford Univ. Press, Oxford, 2006.

[Ka] S. Kamada, Braid and knot theory in dimension four, Math. Surv. and Mon. 95, AMS, Providence, RI, 2002.

[Kw] Y. Kawamata, D-equivalence and $K$-equivalence, J. Differential Geom. 61 (2002), 147-171, arXiv:math.AG/0205287. 
[Kh1] M. Khovanov, A functor-valued invariant of tangles, Algebr. Geom. Topol. 2 (2002), 665-741 (electronic), arXiv:math.QA/0103190.

[Kh2] M. Khovanov, An invariant of tangle cobordisms, Trans. Amer. Math. Soc. 358 (2006), 315-327, arXiv:math.QA/0207264.

[Kh3] M. Khovanov, Crossingless matchings and the cohomology of $(n, n)$ Springer varieties, Commun. Contemp. Math. 6 (2004), 561-577, arXiv:math.QA/0202110.

[Kh4] M. Khovanov, sl(3) link homology I, Algebr. Geom. Topol. 4 (2004), 10451081 (electronic), arXiv:math.QA/0304375.

[Kh5] M. Khovanov, Triply-graded link homology and Hochschild homology of Soergel bimodules, preprint, arXiv:math.GT/0510265.

[KMS] M. Khovanov, V. Mazorchuk, and C. Stroppel, A categorification of integral Specht modules, preprint, arXiv:math.RT/0607630.

[KR] M. Khovanov and L. Rozansky, Matrix factorizations and link homology, preprint, arXiv:math.QA/0401268.

[KS] M. Khovanov and P. Seidel, Quivers, Floer cohomology, and braid group actions, J. Amer. Math. Soc. 15 (2002), 203-271, arXiv:math.QA/ 0006056.

[K] M. Kontsevich, Homological Algebra of Mirror Symmetry, International Congress of Mathematicians (Zürich 1994), Birkhäuser, Basel, 1995, arXiv:alg-geom/9411018.

[MV] M. Mackaay and P. Vaz, The universal $\mathfrak{s l}_{3}$-link homology, preprint, arXiv:math.GT/0603307.

[Ma1] C. Manolescu, Nilpotent slices, Hilbert schemes, and the Jones polynomial, Duke Math. J. 132 (2006), 311-369, arXiv:math.SG/0411015.

[Ma2] C. Manolescu, Link homology theories from symplectic geometry, Adv. in Math. 211 (2007), 363-416, arXiv:math.SG/0601629.

[MS1] V. Mazorchuk and C. Stroppel, Translation and shuffling of projectively presentable modules and a categorification of a parabolic Hecke module, Trans. Amer. Math. Soc. 357 (2005), 2939-2973.

[MS2] V. Mazorchuk and C. Stroppel, On functors associated to a simple root, preprint, arXiv:math.RT/0410339.

[Nk] H. Nakajima, Instantons on ALE spaces, quiver varieties, and Kac-Moody algebras, Duke Math. J. 76 (1994), 365-416.

[Nm] Y. Namikawa, Mukai flops and derived categories, J. Reine Angew. Math. 560 (2003), 65-76, arXiv:math.AG/0203287.

[Pe] T. Perutz, Lagrangian matching invariants for fibred four-manifolds. I, Geom. Topol. 11 (2007), 759-828, arXiv:math.SG/0606061.

[Pl] D. Ploog, Autoequivalences of derived categories of smooth projective varieties, Ph.D. thesis, Berlin (2005).

[Ro1] R. Rouquier, Categorification of the braid groups, preprint, arXiv: math.RT/0409593. 
[Ro2] R. Rouquier, Catégories dérivées et géométrie birationnelle, Séminaire Bourbaki, Astérisque 307 (2006), Exp. No. 946, 283-307, arXiv:math.AG/0503548.

[Ro3] R. Rouquier, Categorification of $\mathfrak{s l}_{2}$ and braid groups, in Trends in Representation Theory of Algebras and Related Topics, 137-167, Contemp. Math. 406, AMS, Providence, RI, 2006.

[RZ] R. Rouquier and A. Zimmermann, Picard groups for derived module categories, Proc. London Math. Soc. 87 (2003), 197-225.

[Ru] L. Rudolph, Braided surfaces and Seifert ribbons for closed braids, Comment. Math. Helv. 58 (1983), 1-37.

[Se1] P. Seidel, unpublished notes (1997).

[Se2] P. Seidel, Lagrangian two-spheres can be symplectically knotted, J. Differential Geom. 52 (1999), 147-171, arXiv:math.DG/9803083.

[Se3] P. Seidel, Graded Lagrangian submanifolds, Bull. Soc. Math. France 128 (2000), 103-149, arXiv:math.SG/9903049.

[Se4] P. Seidel, A long exact sequence for symplectic Floer cohomology, Topology 42 (2003), 1003-1063, arXiv:math.SG/0105186.

[Se5] P. Seidel, Lectures on four-dimensional Dehn twists, preprint, arXiv:math.SG/0309012.

[Se6] P. Seidel, Fukaya categories and Picard-Lefschetz theory, ETH Lecture Notes Series, to appear, European Math. Soc. Draft book available at http://www.math. uchicago.edu/ $\sim$ seidel/draft.pdf.

[SS] P. Seidel and I. Smith, A link invariant from the symplectic geometry of nilpotent slices, Duke Math. J. 134 (2006), 453-514, arXiv:math.SG/ 0405089.

[ST] P. Seidel and R. P. Thomas, Braid group actions on derived categories of sheaves, Duke Math. J. 108 (2001), 37-108, arXiv:math.AG/0001043.

[So] W. Soergel, The combinatorics of Harish-Chandra bimodules, J. Reine Angew. Math. 429 (1992), 49-74.

[St1] C. Stroppel, Categorification of the Temperley-Lieb category, tangles and cobordisms via projective functors, Duke Math. J. 126 (2005), 547-596.

[St2] C. Stroppel, TQFT with corners and tilting functors in the Kac-Moody case, preprint, arXiv:math.RT/0605103.

[Sz1] B. Szendröi, Diffeomorphisms and families of Fourier-Mukai transforms in mirror symmetry, in Applications of Algebraic Geometry to Coding Theory, Physics and Computation (Eilat, 2001), 317-337, NATO Sci. Ser. II Math. Phys. Chem. 36, Kluwer Acad. Publ., Dordrecht, 2001, arXiv:math.AG/0103137.

[Sz2] B. Szendröi, Artin group actions on derived categories of threefolds, J. Reine Angew. Math. 572 (2004), 139-166, arXiv:math.AG/0210121.

[To] B. Toën, The homotopy theory of dg-categories and derived Morita theory, Invent. Math. 167 (2007), 615-667, arXiv:math.AG/0408337. 
Homology, Homotopy and Applications, vol. 9(2), 2007

Mikhail Khovanov khovanov@math.columbia.edu

Department of Mathematics

Columbia University

New York. NY 10027

USA

Richard Thomas richard.thomas@imperial.ac.uk

Department of Mathematics

Imperial College

London. SW7 2AZ

UK

This article is available at http://intlpress.com/HHA/v9/n2/a2 